\title{
Space-time adaptive processing with multi-stage Wiener filter and principal component signal dependent algorithms
}

\author{
A Thesis Presented to the \\ Electrical Engineering Department Faculty of \\ California Polytechnic State University \\ San Luis Obispo, California \\ In Partial Fulfillment \\ Of the Requirements for the Degree of \\ Master of Science in Electrical Engineering
}

By

Zheng Ning Zhou

March 2010 
(C) 2010

Zheng Ning Zhou ALL RIGHTS RESERVED 


\section{COMMITTEE MEMBERSHIP}

TITLE:

Space-time adaptive processing with multi-staged Wiener filter and principal component signal dependent algorithms

AUTHOR: $\quad$ Zheng Ning Zhou

DATE SUBMITTED: April 2010

COMMITTEE CHAIR: John Saghri, Associate Professor

COMMITTEE MEMBER: Helen Yu, Associate Professor

COMMITTEE MEMBER: Jane Zhang, Associate Professor 


\section{Abstract}

\section{Space-time adaptive processing with multi-stage Wiener filter and principal component signal dependent algorithms}

By

Zheng Ning Zhou

Space-time Adaptive Processing (STAP) is a two-dimensional filtering technique for antenna array with multiple spatial channels. The name "space-time" describes the coupling of these spatial channels with pulseDoppler waveforms. Applications for STAP includes ground moving target indicator (GMTI) for airborne radar systems.

Today, there are strong interests to develop STAP algorithms for operations in "sample starved" environments, where intense environmental interference can reduce STAP capacity to detect and track ground targets. Careful applications of STAP can effectively overcome these conditions by suppressing these interferences and maximize the signal to interference plus noise ratio (SINR). The Multi-stage Wiener filter (MWF) and principal component signal dependent (PC-SD) algorithm are two such methods that can suppress these interference through truncation of the signal subspace.

This thesis makes contribution in several ways. First it details the importance of rank compression and sample compression for effective STAP operations in "sample starved" environments. Second, it shows how MWF and PC-SD could operate in this type of environment. Third it details how a "soft stop" technique like diagonal loading (DL) could improve STAP performance in target detection for MWF and PC-SD. Fourth, this thesis contrasts the performance of several existing "hard stop" techniques in rank compression and introduces a new one using a-priori knowledge. 


\section{ACKNOWLEDGEMENTS}

This thesis is made possible by the generous support and advice of Dr John Saghri. I would also like to express my gratitude to Raytheon Space and Airborne Systems for their support of the ongoing synthetic aperture radar (SAR) research project at California Polytechnic State University at San Luis Obispo.

I would also like to thank Dr Helen Yu and Dr Jane Zhang for their participation as members of the thesis committee.

I thank other members of Cal Poly's SAR team including John Hupton, Jessica Kiefer, Scott Seims and Matthew Schlutz for their work and advice.

Finally I want to thank my family, especially my mom for her unending love and support. 


\section{Table of contents}

List of Figures .......................................................... vii

List of Tables ................................................................ ix

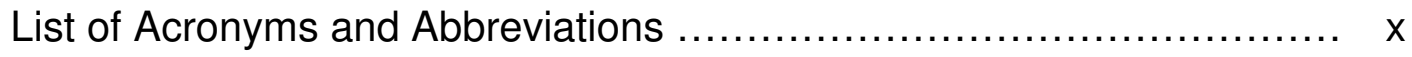

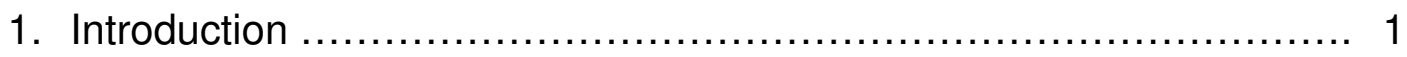

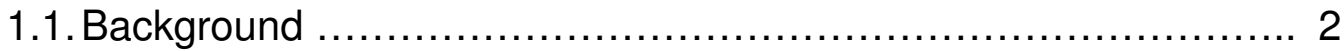

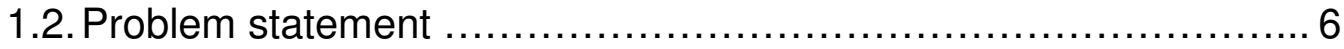

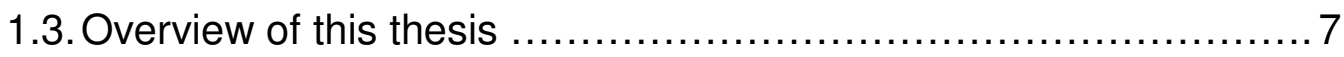

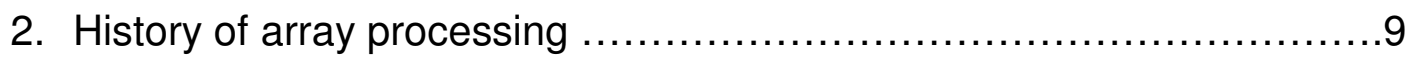

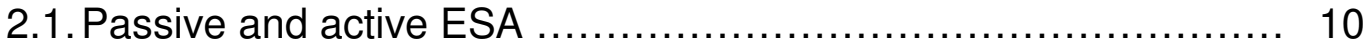

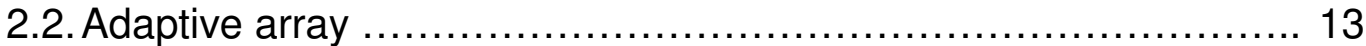

2.3. Signal models ..................................................... 14

2.3.1. Desired target signal ...................................... 15

2.3.2. Noise signal ............................................ 18

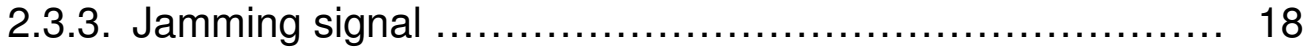

2.3.4. Signal to noise ratio ..................................... 19

2.3.5. Jammer to noise ratio ................................... 19

2.4. Beam-forming fundamentals .................................... 20

2.4.1. Minimum variance distortion-less response .................. 21

2.4.2. Adaptive beam-forming ...................................... 24

2.4.3. Performance metrics ....................................... 26

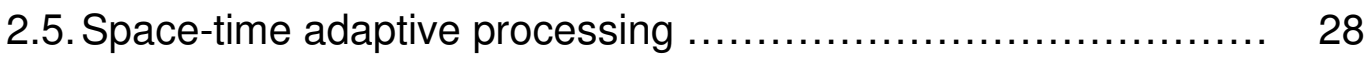

2.5.1. Clutter .................................................. 32

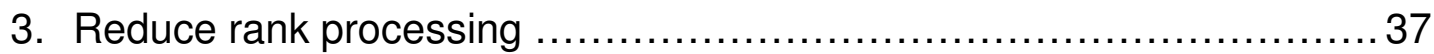

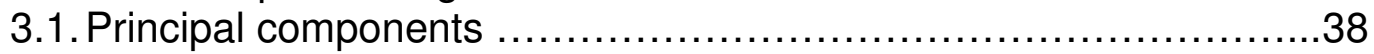

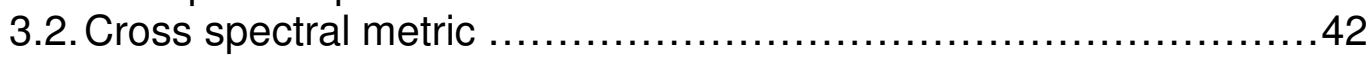

3.3. Multi-stage Wiener filter ........................................ 43

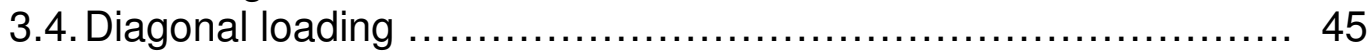

3.5. Relationships between DL, PC and MWF ..................... 47

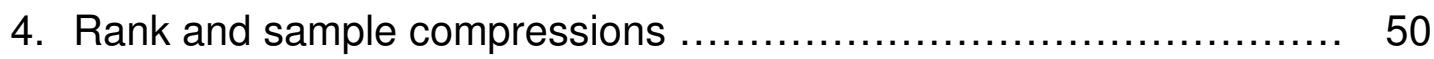

4.1. Sample compression ............................................. 51

4.2. Rank compression ............................................. 55

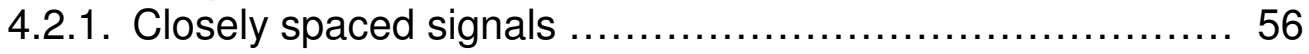

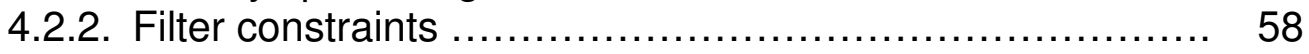

4.2.3. Pattern constraints ........................................ 59

4.2.4. Rank compression in MWF ............................... 60

4.2.5. Practical factors to consider ................................ 62

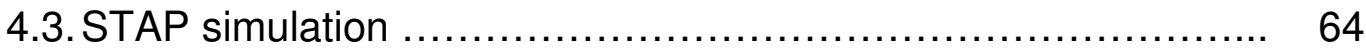

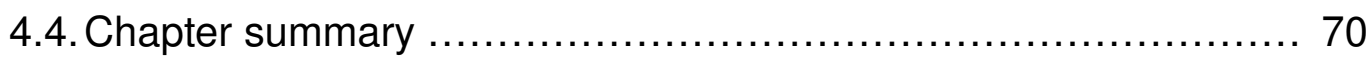




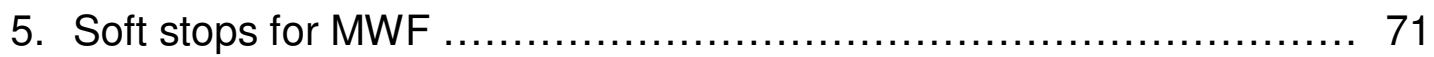

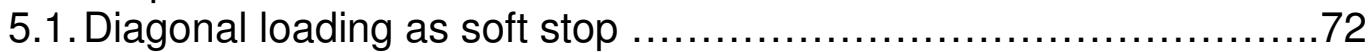

5.2. Diagonal loading examples ........................................ 73

5.2.1. Finding the right loading level ............................. 75

5.3. Chapter summary .................................................. 75

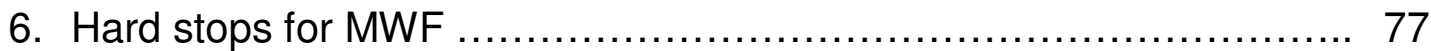

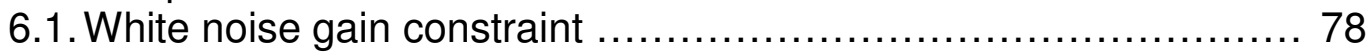

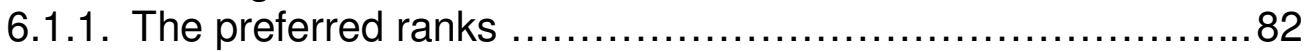

6.1.2. Histogram of rank selection ................................. 83

6.2. Modified Hanke-Raus error estimation ............................. 87

6.2.1. The Hanke-Raus error estimation ......................... 87

6.3. MHREE with diagonal loading …................................ 94

6.4. Taxonomies of hard stop techniques ................................ 101

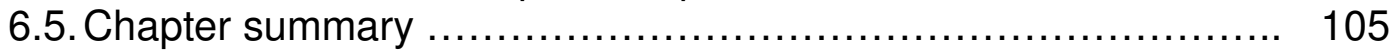

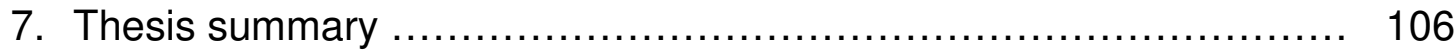

Bibliography ......................................................... 108

Appendix A: Matlab codes for MWF vs PC-SD MSE performance ........ 114

Appendix B: Matlab codes for study of SINR vs sample support .......... 122

Appendix C: Matlab codes for synthesizing SAR image ................... 129

Appendix D: Matlab codes for the studying of CMT effects ................ 134

Appendix E: Matlab codes for histogram study of hard stops .............. 138 


\section{List of figures}

$1 \quad$ The classic Wiener filter......................................... 3

2 Block diagram of adaptive array filter.............................. 4

$3 \quad$ Reduce Rank Transformation in block diagram................... 5

$4 \quad$ Passive ESA architecture....................................... 10

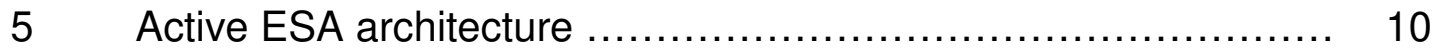

$6 \quad$ ESA beam-former …................................................... 11

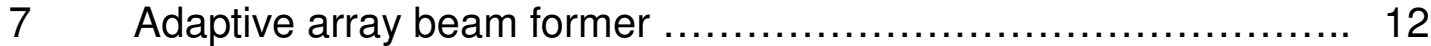

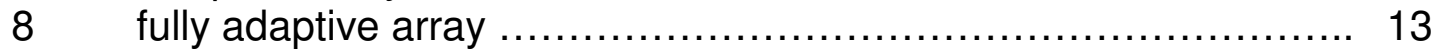

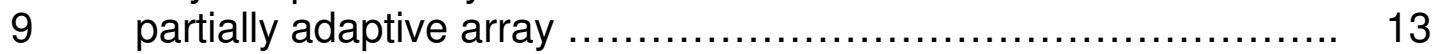

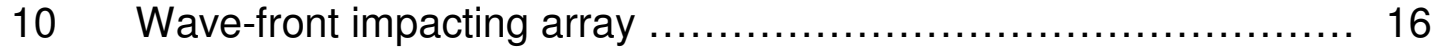

11 Channelization of wideband signal ............................. 17

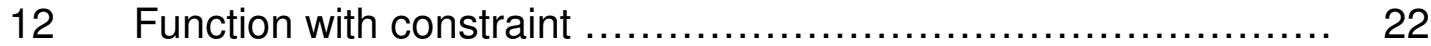

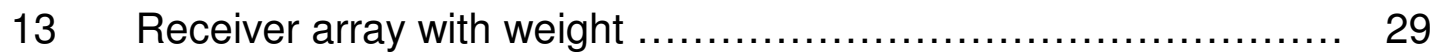

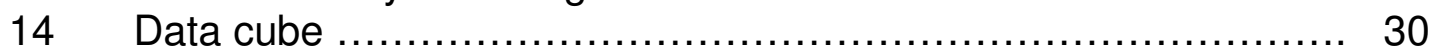

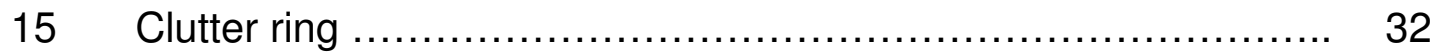

$16 \quad$ Clutter "ridge" ................................................ 33

$17 \quad$ Top view of clutter ridges of various $\beta$ values ..................... 34

$18 \quad$ Reduce rank transformation .................................... 37

19 Principal component signal independent ….................... 40

20 Principal component signal dependent .......................... 41

$21 \quad$ A three stage adaptive filter ....................................... 44

$22 \quad$ Normalized SINR for two jammers ............................... 54

$23 \quad$ Normalized SINR for three jammers ........................... 54

$24 \quad$ Classic Wiener filter ............................................ 55

$25 \quad$ General side-lobe canceller ................................... 58

26 beam-former with Chebychev taper ............................. 59

$27 \quad$ Eigenspectra ................................................... 64

$28 \quad$ MWF vs PC-SD w/ 50dB JNR and 10dB CNR .................. 67

$29 \quad$ MWF vs PC-SD various JNR and 10dB CNR ................... 68

$30 \quad$ MWF vs PC-SD w/ 50dB JNR and various CNR .................. 69

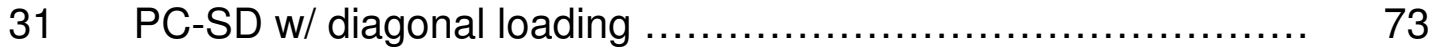

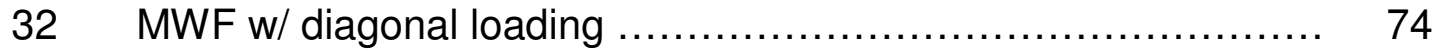

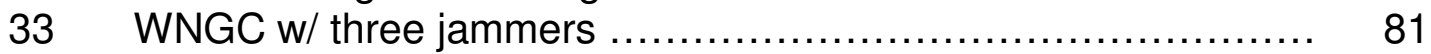

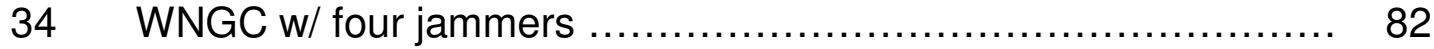

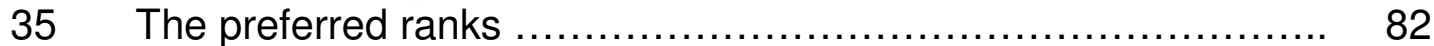

$36 \quad$ Example of WNGC rank selection ............................... 84

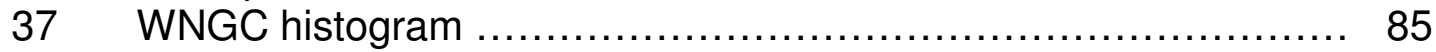

$38 \quad$ Example of WNGC over-thresholding ............................ 86

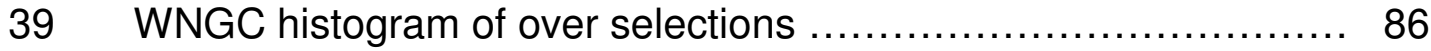

$40 \quad$ Basic filter ....................................................... 87

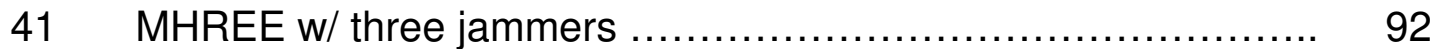

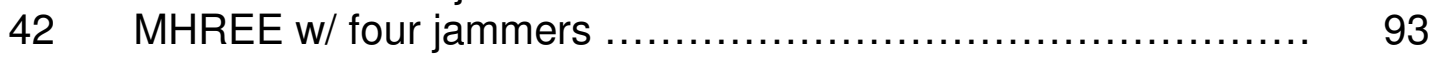

43 MHREE and diagonal loading $w /$ three jammers .................. 94 


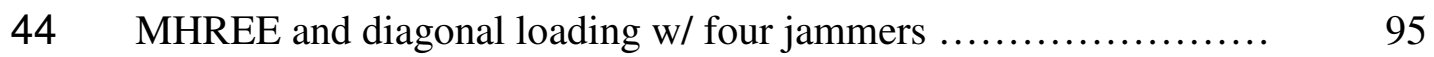

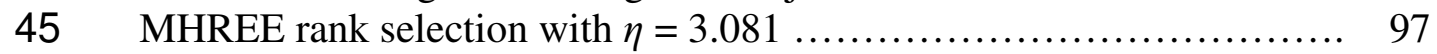

46 Histogram of MHREE rank selection with $\eta=3.081 \ldots \ldots \ldots \ldots \ldots \ldots . . . \ldots 7$

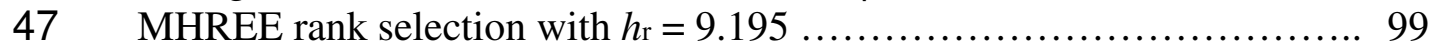

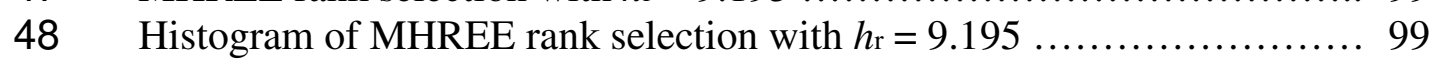

49 Basic filter ............................................... 101 


\section{List of tables}

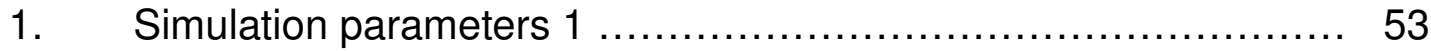

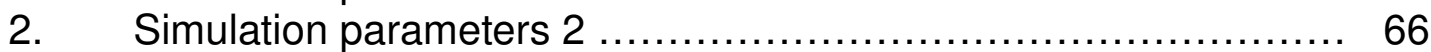

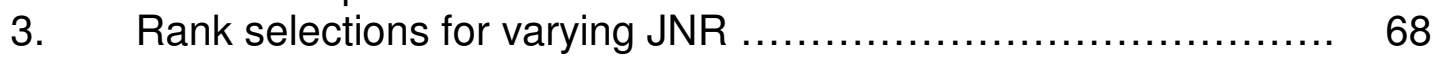

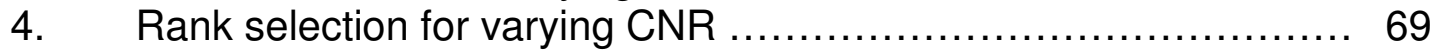

5. 10 examples of Optimum thresholds for WNGC .................. 83

6. Translation of filter parameters ................................ 89

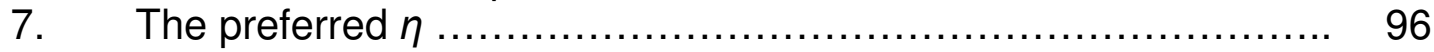

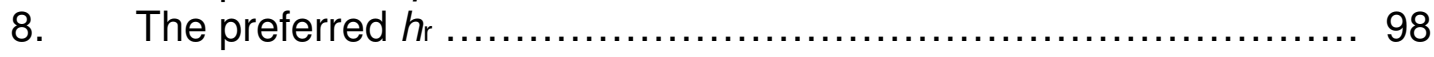

9. Best $h r$ thresholds for various numbers of jammers and jammer

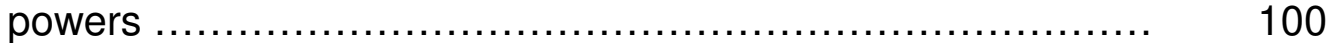

10. Taxonomy of "hard stop" techniques based on filter parameters ... 102

11. Taxonomy of "hard stop" techniques based on threshold. .......... 104 


\section{ACRONONYM AND ABBREVIATIONS}

\begin{tabular}{|c|c|}
\hline$A / D$ & Analog-to-digital converter \\
\hline AIC & Akaike Information Criterion \\
\hline CG & Conjugate Gradients \\
\hline CGLS & Conjugate Gradient Least Squares \\
\hline CMT & Covariance Matrix Taper \\
\hline CPI & Coherent Processing Interval \\
\hline CSM & Cross Spectral Metric \\
\hline CV & Cross Validation \\
\hline$d B$ & Decibel \\
\hline DL & Diagonal Loading \\
\hline ESA & Electronically Scanned Array \\
\hline EVD & Eigenvalue Decomposition \\
\hline GDP & Generalized Discrepancy Principle \\
\hline GSC & Generalized Sidelobe Canceller \\
\hline HREE & Hanke-Raus Error Estimate \\
\hline IID & Independent and Identically Distributed \\
\hline IOPF & Input-Output Power Function \\
\hline ISMSE & Independent Sample Mean Square Error \\
\hline JNR & Jammer-to-Noise Ratio \\
\hline LNR & Loading-to-Noise Ratio \\
\hline MDL & Minimum Description Length \\
\hline MHREE & Modified Hanke-Raus Error Estimate \\
\hline MMSE & Minimum Mean Square Error \\
\hline MSE & Mean Square Error \\
\hline MVDR & Minimum Variance Distortionless Response \\
\hline MWF & Multi-stage Wiener Filter \\
\hline PC & Principal Components \\
\hline PC-SD & Signal Dependent Principal Components \\
\hline PC-SI & Signal Independent Principal Components \\
\hline PRF & Pulse Repetition Frequency \\
\hline SINR & Signal-to-Interference-plus-Noise Ratio \\
\hline SMI & Sample Matrix Inversion \\
\hline SMSE & Sample Mean Square Error \\
\hline SNR & Signal-to-Noise Ratio \\
\hline STAP & Space Time Adaptive Processing \\
\hline SVD & Singular Value Decomposition \\
\hline ULA & Uniform Linear Array \\
\hline & White Noise Gain Constraint \\
\hline
\end{tabular}




\section{Chapter 1}

\section{Introduction}

First published in the 1970s by Lawrence E. Brennan and Irving S. Reed, Space-Time Adaptive Processing (STAP) is a signal processing technique mostly used in radar systems to aid in target detection. It uses adaptive processing algorithms to suppress interferences such as jamming, ground clutter and noise to increase system sensitivity to target returns.

STAP commonly uses a phased-array antenna with multiple elements (i.e. spatial channels). The term "space-time" derives from the coupling of these spatial channels with pulse Doppler waveforms thus creating a two dimensional filter to suppress stated interferences. Adding the statistics of interference environment (which can be known or unknown), an adaptive STAP weight vector can be estimated to improve target detection.

A common application is an airborne STAP platform used in detecting ground moving targets, also known as ground moving target indicator (GMTI). One purpose of this thesis is to demonstrate how adaptive filtering of target signals can be achieved via multi-stage Wiener filter (MWF) and Principal Component signal dependent (PC-SD) algorithms. 
This thesis will also demonstrate how MWF performance can be improved with techniques such as diagonal loading (DL) and modified Hanke-Raus error estimation (MHREE) technique. Simulations will later show that MWF generally offer superior rank and sample support compression than the more commonly used PC-SD.

This thesis makes contributions in several areas. First it details how MWF and PC contribute to rank and sample support compression. Second it shows how diagonal loading can be added to improve MWF and PC performances. Lastly it shows how hard stop techniques like modified Hanke-Raus error estimation (MHREE) can reduce processing time required for the MWF.

\section{$1.1 \quad$ Background}

MWF is an adaptive filtering algorithm that seeks to minimize the mean square error (MSE). More precisely it seeks to derive a weight vector (a user controllable parameter) that can yield the highest possible signal to interference plus noise ratio (SINR). This will yield the highest probability of target detection. The stated signal power is the received target returns, and the interference plus noise are self explanatory. In most environments and applications, target signal returns are very weak. This can be due to many factors such as low transmission power, array gain, target size, 
target range, interferences, etc. In realm of signal processing we cannot alter most of these factors; however, we can effectively reduce the impact of interferences to increase target detection. Interferences contaminate our desired signal returns and can be effectively suppressed. One technique to accomplish this is to use the Wiener filter (WF).

The original Wiener filter is a linear, discrete time, adaptive finite impulse response filter. It is the fundamental building block of linear adaptive filtering

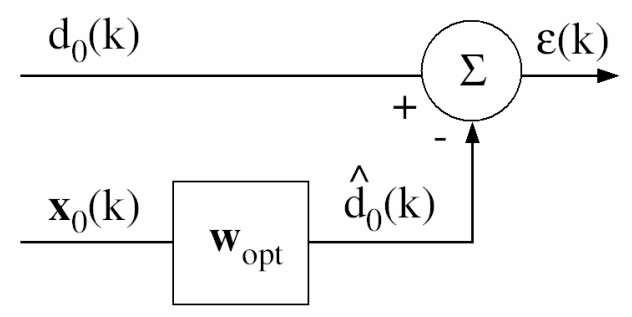

Figure 1: The classic Wiener filter

Figure 1 shows a block diagram of the classic Wiener filter where $d_{0}(k)$ and $\hat{d}_{0}(k)$ are scalars. The output minimizes the mean square error by subtracting a weighted signal $\mathbf{x}_{0}$ from the desired signal $\mathbf{d}_{0}$ or

$$
M S E=\mathbf{E}\left[\left|\mathbf{d}_{0}(k)-\mathbf{w}^{H} \mathbf{x}_{0}(k)\right|^{2}\right]=\sigma_{d_{0}}^{2}-\mathbf{w}^{H} \mathbf{r}_{x_{0} d_{0}}-\mathbf{r}_{x_{0} d_{0}}^{H} \mathbf{w}+\mathbf{w}^{H} \mathbf{R}_{x_{0}} \mathbf{w}
$$

where

\section{Equation 1.1}

$$
\mathbf{R}_{x_{0}}=\mathbf{E}\left[\mathbf{x}_{0} \mathbf{x}_{0}^{H}\right]
$$

Equation 1.2

$$
\mathbf{r}_{x_{0} d_{0}}=\mathbf{E}\left[\mathbf{x}_{0} \mathbf{d}_{0}^{*}\right]
$$

Equation 1.3

and 


$$
\sigma_{d_{0}}^{2}=\mathbf{E}\left[\mathbf{d}_{0} \mathbf{d}_{0}^{*}\right]
$$

Equation 1.4

If we take the gradient of both sides of Equation 1.1 with respect to the weight vector $\mathbf{w}$ and set it equal to 0 , we get

$$
\nabla_{w}(M S E)=-\mathbf{r}_{x_{0} d_{0}}+\mathbf{R}_{x_{0}} \mathbf{w}=0
$$

Equation 1.5

This leads us to the classic Wiener filter equation:

$$
\mathbf{w}_{\text {opt }}=\mathbf{R}_{x_{0}}^{-1} \mathbf{r}_{x_{0} d_{0}}
$$

Equation 1.6

In Equation 1.6, the weight vector is dependent on the given environment; hence it is "adaptive". Though this equation seems relativity simple in theory, it's a different story in practice. Both $\mathbf{R}$ and $\mathbf{r}$ are actually unknown and are estimated from acquired samples of the environment. Due to this inconvenient truth, system performance is heavily dependent on sample support.

Today, Application for Wiener filter includes interference cancellation, equalization, and multisensory array processing, which is the focus of this thesis.

One of the most interesting forms of multisensory array processing is the adaptive array shown in figure 2. 


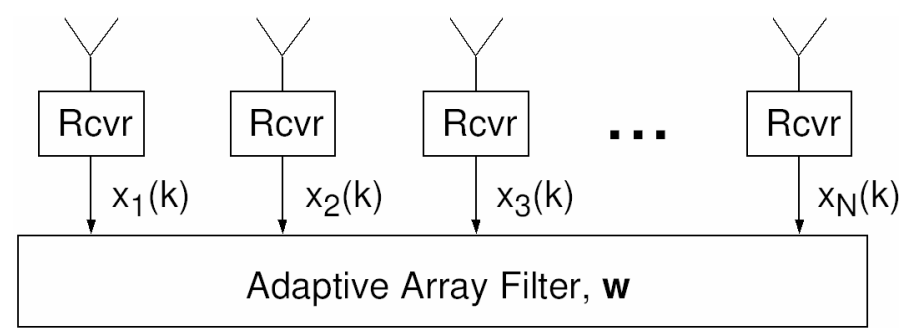

Figure 2: Block diagram of adaptive array filter

Adaptive array filter collects data samples from each spatial channel (element receiver) and jointly process the information to acquire useful knowledge of the environment.

In practice, large arrays are generally impractical due to its computational burden, cost and sample support requirements in weight training, therefore spawning motivations for partial adaptive array processing.

Partial adaptive array utilizes a reduced rank transform (RRT) on the collected data to reduce its adaptive degree of freedom (DOF). This reduces the computational complexities and training sample requirements.

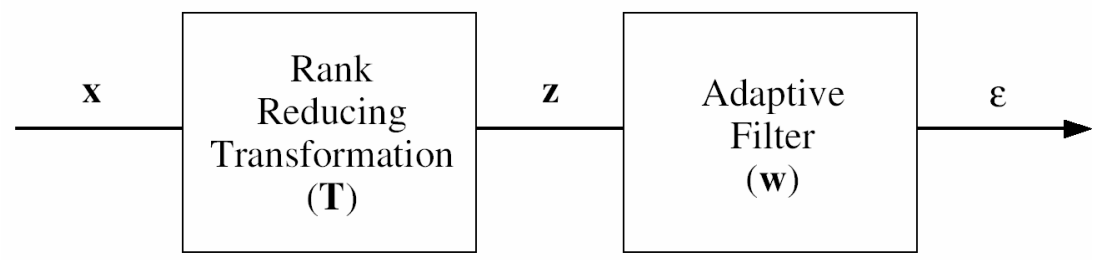

Figure 3: Reduce Rank Transformation in block diagram

Both principal component signal dependent (PC-SD) and multistage Wiener filter (MWF) are reduced rank transformations (shown in Figure 3), but they use different methods to accomplish this goal. 
Many known techniques for reduce rank processing such as PC and cross spectral metric (CSM) uses statistical transformation that transforms element-based data (output of array elements) to eigenspace beamformer. MWF is another example of statistical reduce rank algorithm but can operate in a more compact subspace than PC and CSM. This results to superior rank compression. Though this rank compression is not apparent for spatial array processing (SAP), it is very noticeable in spacetime adaptive processing (STAP).

The motivation for many STAP derived algorithms is to reduce system complexity (hence cost) while improve performance in target detection. Translating these objectives into design goals would result into the following:

1. Reduce computation requirement for STAP

2. Reduce training sample requirements

3. Increase performance in target detection

\subsection{Problem Statement}

This thesis is derived from Raytheon's University Research project with CalPoly on SAR image target tracking. The intent of this project is to expose CalPoly students to SAR and its applications. For example, my predecessor Jessica Kiefer compared target centroid tracking performances of the Kalman and $\mathrm{H}$ Inifinity filter on Moving and Stationary Target Acquisition and Recognition (MSTAR) images. 
Though SAR image tracking has much application appeal and performed admirably, it's not without shortcomings. Disadvantages to tracking with SAR image processing include delays from image synthesizing, overcoming typical image distortion effects and need for classifiers. For example, typical SAR image may need 3 to 5 seconds to be synthesized, therefore all target identification and tracking algorithms needs to wait at least that long. These factors create an environment where critical information is not produced in "real-time" which is crucial for fast decision making. This thesis will explore an alternative - space-time adaptive processing (STAP).

First a few clarifications are needed. STAP makes no attempt at forming images nor does it classify targets. It is capable of detecting target returns with information on target range and direction. It has many desirable traits: first, it is adaptive, meaning that it can function in any environment because it adapts to the environment. Second, STAP is faster than most alternatives such as SAR image tracking because it does not require heavy computations. Third, it is relativity simple meaning that it requires no specialized hardware. Fourth it is versatile to implement, meaning it can work using a variety of algorithms (such as PC and MWF) on many platforms ranging from space satellites to a small low flying unmanned aerial vehicle (UAV). 
This thesis will present a general walkthrough of STAP followed by descriptions of how RRT like PC and MWF impact STAP in practice. Afterward I will detail why the MWF is better than PC in accomplishing our stated goals. Later I will detail how augmenting techniques like diagonal loading (DL) and modified Hanke-Raus error estimation (MHREE) technique improves MWF performance for STAP.

\subsection{Overview of this thesis}

This thesis is broken down into seven chapters. Chapter 2 will discuss adaptive array processing. It covers many historical facts relevant to this thesis and details how STAP came to be. Chapter 3 discusses the significance of reduce rank transformations (RRT). RRT such as PC, CSM and MWF will be translated from theory into practice.

Chapter 4 will chronicle the importance of rank and sample support compression for STAP. This chapter also details a few "natural" factors that contribute to rank compression. It also includes MATLAB simulations that will contrast PC and MWF performance.

Chapter 5 explains how a "soft stop" technique like diagonal loading (DL) improves MWF mean square error (MSE) performance. Additional simulations will demonstrate these improvements. 
Chapter 6 details how "hard stop" techniques like white noise gain constraint (WNGC) and modified Hanke-Raus error estimation (MHREE) improves MSE performance. Four other "hard stop" techniques will also be referenced. A comparison of these techniques will be conducted. Chapters 7 summarizes this thesis and proposes future work. 


\section{Chapter 2}

\section{History of array processing}

In the early days, mechanically scanned arrays operated in frequency range from 30 to $200 \mathrm{MHZ}$ like the US Army's $100 \mathrm{MHz}$ SCR-270 that detected the Japanese attack on Pearl Harbor. That array was a collection of Half-wave dipoles that generated a broadside main beam to scan nearby airspace for planes.

Once the radar frequency moved into the microwave region, antenna arrays were dropped in favor of parabolic reflector. In the 1970s, development of waveguide radiator technologies made it possible for antenna arrays to have ultra low side lobes in microwave frequencies. This created a resurgence of interest into antenna arrays. A modern day example of that would be the AWACS (Airborne Warning and Control System) used by the U.S. Air Force.

Though the antenna array had low side lobes it was still very limited. Modern military applications demands faster response time and more flexibility than what a mechanically scanned array allowed. Therefore interests rose for electronically scanned arrays (ESA). 
In the 1960s, development of solid state devices like phase shifters made it possible for antenna arrays to have very fast and flexible beam forming architecture. Hence giving birth to electronically scanned arrays (or phased arrays). These usually appeared for some more demanding military applications such as an instance where a single array must perform multiple functions simultaneously such as tracking and surveillance. Beam steering is accomplished by altering the respective phase of electrical current fed to individual elements, instead of mechanical apparatuses like gimbals, motors and joints.

At introduction, electronically scanned array (ESA) were complex and prohibitive costly. Advancement of computer processing technology alleviated some of that cost and therefore ESA are now much more widespread.

\subsection{Passive and active ESA}

Figure 4 shows the architecture of a passive electronically scanned array (ESA). It contains phase shifters at each element to facilitate beam forming. The transmitter and receiver are centrally located. The feed block can feed to and from the array and it can perform a constrained feed like a waveguide or a space feed. In a passive ESA, its transmitters 
and amplifiers are centrally located therefore its phase shifters must be able to handle high power.

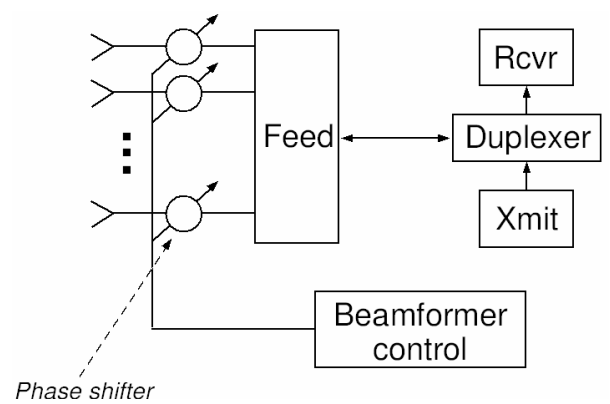

Figure 4: Passive ESA architecture

Figure 5 shows generic blocks of an active ESA. Its architecture is dubbed the distributed transmitter architecture, which means each element contains a transmitter/receiver (T/R) module that provides amplification. In addition, the T/R module also has a phase shifter and a Iow noise amplifier. The active ESA retains a centralized receiver.

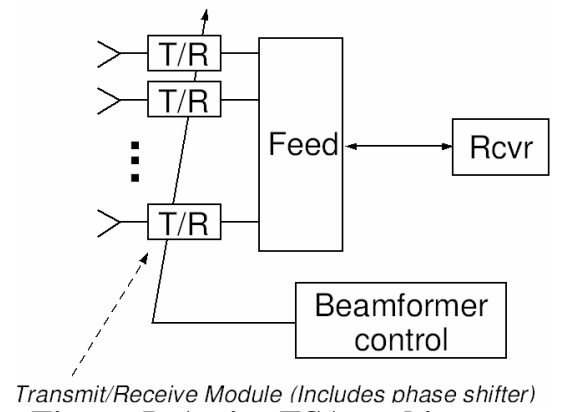

Figure 5: Active ESA architecture

Though ESA is much faster and more flexible than mechanically scanned array, it still lacked when dealing with one important real world problem: interferences. Interferences are mostly jammer sources, noise, and clutter (in STAP only). These distort the desired signal returns therefore making target detection difficult. Jammers are typically considered 
stationary omni-directional transmitters, clutters are the unwanted echoes of our transmitted signals and noise is the thermal noise of elements.

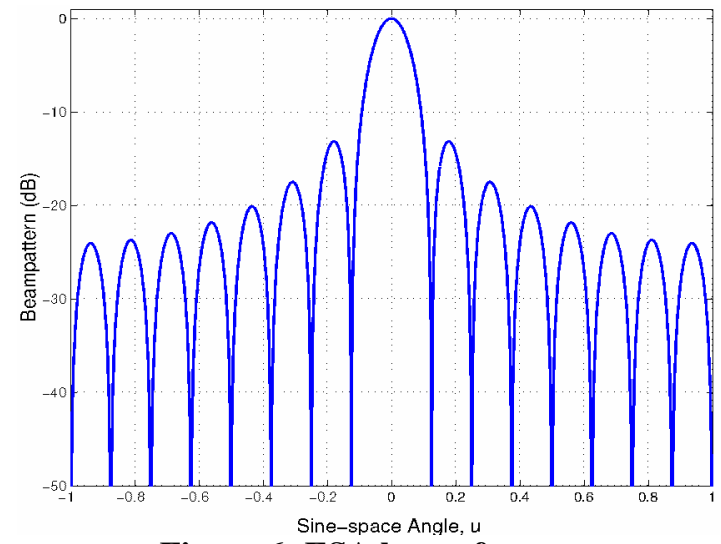

Figure 6: ESA beam-former

Figure 6 shows an example beam former of ESA. It does not adapt to interferences thus performs poorly in environments where jammers exists. Hence we need a beam former that can adapt to the environment.

An adaptive array electronically steers not only the beams but also the nulls as shown in Figure 7.

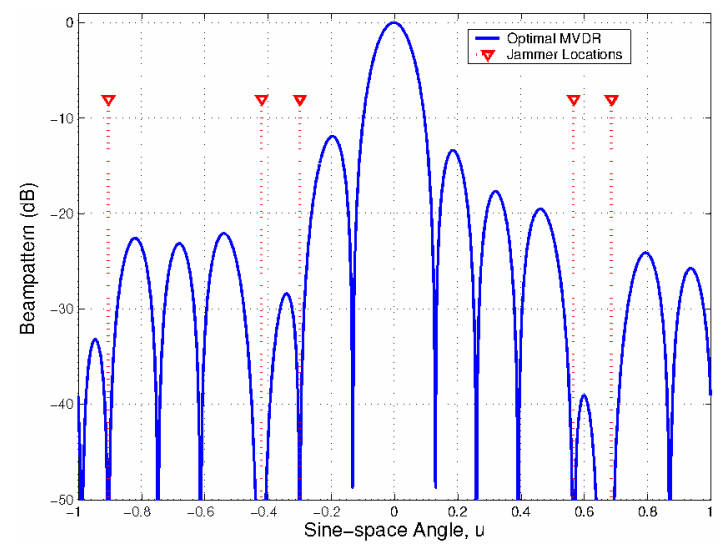

Figure 7: Adaptive array beam-former 
These nulls are steered toward the directions of the jammers and nullify its detrimental effects. Adaptive arrays typically first sample the environment to estimate the environmental interferences. A weight vector can then be calculated to modify the side lobes for effective null steering and suppress the interferences. Once these interferences are effectively suppressed, we can maximize the signal to interference plus noise ratio (SINR) and find our targets. Because of its ability to adapt to any environment, adaptive arrays are now a very attractive option for applications in interference heavy environments.

\subsection{Adaptive array}

There are generally two types of adaptive arrays: fully adaptive and partially adaptive.

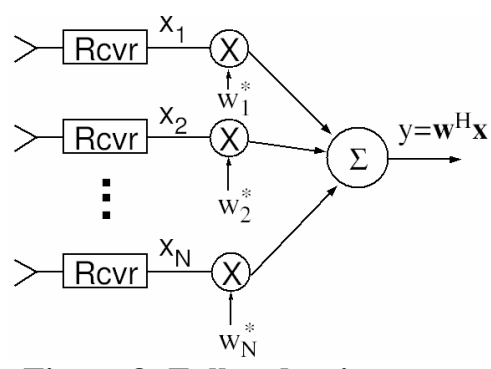

Figure 8: Fully adaptive array

Figure 8 shows the receive-only version of a fully adaptive array. Each receive element of the array has an analog to digital (A/D) converter. 
Their outputs are then individually multiplied by a pre-calculated complex weight. The results are then linearly combined to form the beam former. The number of adaptive degree of freedoms is equal to the number of weights.

A partially adaptive array is similar to a fully adaptive array except that a rank reducing transform is applied to the data beforehand. Figure 9 shows an example.

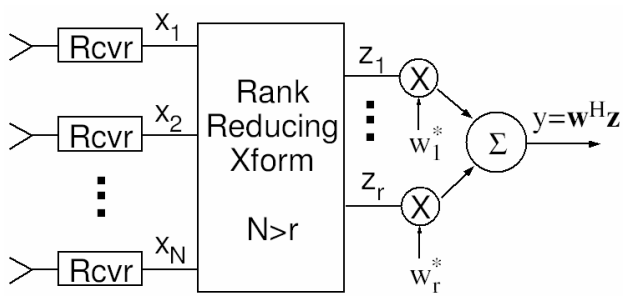

Figure 9: Partially adaptive array

This reduces the number of degree of freedoms in data processing and weight calculation. This reduction lessens the computational burden in data processing, which can be significant due to the typically enormous workload and fast response time in the realm of STAP. Recent and more compelling motivation for using partially adaptive arrays is that they can also provide superior performance than the fully adaptive array for real world applications. For example, in some battlefield conditions, heavy jamming interference makes it difficult to acquire adequate sample support for a fully adaptive array. By contrast, a partially adaptive array needs fewer samples to operate and hence is the more attractive option (18). 
There are generally two ways to implement reduce rank transformations (RRT): sub-arraying, which is considered by most to be costly, or signal processing (48). RRT implemented through signal processing can be done through deterministic approaches like beam space processing or through statistical approaches like principal component and the multistage Wiener filter $(70,77)$. We will discuss these statistical approaches more fully in the next chapter.

\subsection{Signal Models}

Before we get into STAP we must first understand its predecessor, spatial array processing (SAP). In SAP there are generally three types of signals: desired target signal, jammer signal and noise signal. Thus the total signal received by our array is $\mathbf{x}$, which equals the superposition of the three signals.

$$
\mathbf{x}=\mathbf{x}_{s}+\mathbf{x}_{J}+\mathbf{x}_{n}
$$

Equation 2.1

The jammer and noise are classified as interference. This undesired interference signal is

$$
\mathbf{x}_{u}=\mathbf{x}_{J}+\mathbf{x}_{n}
$$

Equation 2.2 
and modeled as a zero mean Gaussian random variable. Therefore the covariance matrix of this undesired signal would be $\mathbf{R}_{u}=\mathbf{E}\left[\mathbf{x}_{u} \mathbf{x}_{u}^{H}\right]=\mathbf{R}_{n}+\mathbf{R}_{J}$. And because these are Gaussian, their covariance matrix fully describes their statistical properties.

\subsubsection{Desired target signal}

Unlike the undesired signals which are random signals with unknown amplitude and phases, the desired signal is a narrowband (i.e. signal frequency bandwidth is small compare to carrier freq) sinusoidal waveform, or

$$
\tilde{x}(t)=\alpha e^{j\left(2 \pi f_{c} t-\phi\right)}
$$

Equation 2.3

where $\alpha$ is the unknown amplitude, $f_{c}$ is the carrier frequency and $\phi$ is the unknown phase shift.

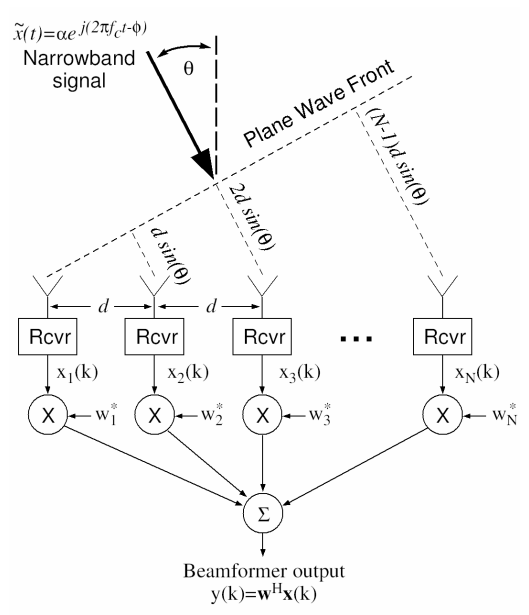

Figure 10: Wave-front impacting array 
Figure 10 shows a wave front impacting a uniform linear array (ULA). For positive values of $\theta$, our wave front first arrive at the left most element. This wave front will then have to travel an additional path length of $d \sin \theta$ in order to reach the next element and so forth. Since this signal is assumed narrowband we can approximate the propagation delay between the elements as phase shifts.

As mentioned earlier, the adaptive array has a receiver behind each element. These receivers digitize the received signal. The combined output of these receivers is an $\mathbf{N}$ dimensional vector in the form of

$$
\mathbf{x}=x\left[e^{j 0}, e^{j 2 \pi \frac{d}{\lambda} \sin \theta}, \ldots e^{j 2 \pi(\mathbf{N}-1) \frac{d}{\lambda} \sin \theta}\right]^{T}=x \mathbf{v}_{\theta}
$$

Equation 2.4

where $x$ is a complex baseband signal received at the left most element and $\mathbf{v}_{\theta}$ is known as the spatial array manifold vector (AMV) given by

$$
\mathbf{v}_{\theta}=\left[e^{j 0}, e^{j 2 \pi \frac{d}{\lambda} \sin \theta}, \ldots e^{j 2 \pi(N-1) \frac{d}{\lambda} \sin \theta}\right]^{T}
$$

Equation 2.5

$T$ is the transpose operator, $d$ is the inter element distance and $\lambda$ is the center frequency wavelength.

One important assumption from above is that the signals are narrowband, or that the signal bandwidth is small compare to the carrier 
frequency. This assumption can be enforced as a design parameter on the some system's operating bandwidth. For other applications where this is not possible, one can still satisfy the narrowband assumption through channelization.

In this channelized processing architecture, a wideband received signal can first be transformed to the frequency domain and then broken down to multiple parallel narrowband beam formers. The output of these beam formers can then be recombined into a single composite beam former output as shown in Figure 11.

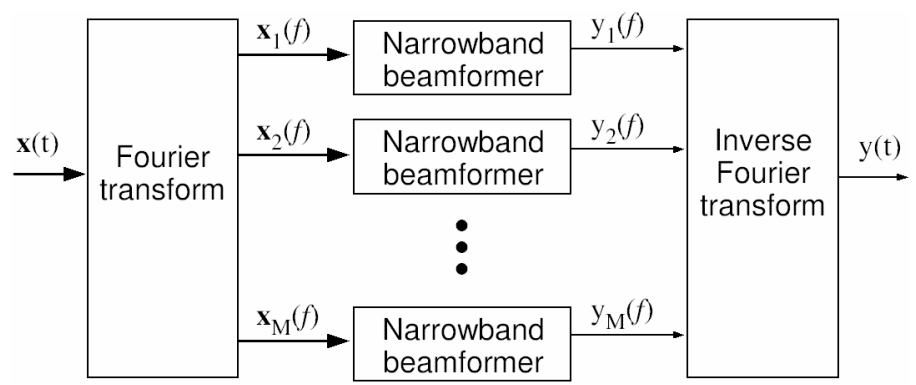

Figure 11: Channelization of wideband signal

\subsubsection{Noise signal}

The first interference we model is noise. In our receiver array, each element produces thermal noise. These thermal noises can be modeled 
as zero mean Gaussian random processes thus uncorrelated with each other. The noise covariance matrix is

$$
\mathbf{R}_{n}=\sigma_{n}^{2} \mathbf{I}
$$

Equation 2.6

where $\mathbf{I}$ is the identity matrix of size of $\mathbf{N} \times \mathbf{N}$ dimension, $\mathbf{N}$ is the total number of elements and $\sigma_{n}^{2}$ is each element's thermal noise power. This noise power is equal to $\sigma_{n}^{2}=k T_{n} B$, where $k$ is the Boltzmann constant, $T_{n}$ is noise temperature (in Kelvin), and $B$ is the receiver bandwidth in $\mathrm{Hz}$. Typically in analysis, the element noise power is normalized to unity for convenience, meaning thermal noise $\sigma_{n}^{2}=k T_{n} B$ is normalized to 1 .

\subsubsection{Jamming signal}

The jammers are modeled as spatial point sources that constantly transmit a high power omni-directional interference signal. This signal's covariance matrix is equal to

$$
\mathbf{R}_{J}=\sigma_{J}^{2} \mathbf{v}_{\theta_{J}} \mathbf{v}_{\theta_{J}}^{H}
$$

\section{Equation 2.7}

where $\sigma_{J}^{2}$ is the jammer's power and $\mathbf{v}_{\theta_{J}}$ is the array manifold vector

associated with jammer's direction of arrival. If dealing with $N_{j}$ jammers, 
their covariance matrix would add because jammers are mutually uncorrelated.

$$
\mathbf{R}_{J, \text { Total }}=\sum_{i=1}^{N_{j}} \mathbf{R}_{J}(i)
$$

Equation 2.8

\subsubsection{Signal to noise ratio}

The signal to noise ratio is a metric that most should be familiar with.

$$
S N R=\frac{\sigma_{s}^{2}}{\sigma_{n}^{2}}
$$

Equation 2.9

The $\sigma_{s}^{2}$ is the received signal power at element level and $\sigma_{n}^{2}$ is thermal power at element level.

\subsubsection{Jammer to noise ratio}

Likewise, the jammer to noise ratio is defined as

$$
J N R=\frac{\sigma_{j}^{2}}{\sigma_{n}^{2}}
$$

Equation 2.10

Similarly, the $\sigma_{j}^{2}$ is the received jammer power at element level and $\sigma_{n}^{2}$ is same as above. Both SNR and JNR are defined at the element level and used to characterize the environment at the input of the array. 
When dealing with $N_{j}$ jammers, the total JNR is equal to

$$
J N R_{\text {Total }}=\frac{\sum_{i=1}^{N_{j}} \sigma_{j}^{2}(i)}{\sigma_{n}^{2}}
$$

Equation 2.11

where $N_{j}$ is again the total number of jammers. Note that SNR and JNR should always be expressed in decibels (dB).

\subsection{Beam forming fundamentals}

The beam former output $y$ of our array at Figure 10 is

$$
y=w_{1}^{*} x_{1}+w_{2}^{*} x_{2} \ldots+w_{N}^{*} x_{N}=x \sum_{i=1}^{N} w_{i}^{*} e^{j 2 \pi(i-1) \frac{d}{\lambda} \sin \theta}=x \mathbf{A}(\theta)
$$

Equation 2.12

where $\mathbf{A}(\theta)=\sum_{i=1}^{N} w_{i}^{*} e^{j 2 \pi(i-1) \frac{d}{\lambda} \sin \theta}$ is the array factor, this can also be considered the beam pattern $\mathbf{B}(\theta)$ since we are treating all element as omnidirectional. But typically in practice, element pattern is not omnidirectional and therefore the beam pattern is the product of array factor and element radiation pattern $\mathbf{B}(\theta)=\mathbf{A}(\theta) \mathbf{B}_{e}(\theta)$.

\subsubsection{Minimum Variance Distortion less Response}


A minimum variance distortion-less response (MVDR) beam former is also known as the optimal beam former which can accomplish two very desirable objectives:

1. Minimize the array output interference power.

2. Get the target desired signal without distortion.

Since our only controllable parameters are the complex weights, we must derive a weight vector $\mathbf{w}$ that can achieve the two objectives. Note that our array output in vector notation is $\mathbf{y}=\mathbf{w}^{H} \mathbf{x}$. This output is a combination of outputted desired signals and interference signals, or

$$
\mathbf{y}=\mathbf{y}_{s}+\mathbf{y}_{i, n}
$$

Equation 2.13

The interference output $\mathbf{y}_{i, n}$ is the sum of noise and jammer outputs or $\mathbf{y}_{i, n}=\mathbf{y}_{n}+\mathbf{y}_{j}$ and $\mathbf{y}_{s}$ is the signal output. If we want to minimize the interference output power then we must minimize $\mathbf{E}\left[\left|\mathbf{y}_{i, n}\right|^{2}\right]$. This can be accomplished by reforming the equation as

$$
\min \left\{\mathbf{E}\left[\left|\mathbf{y}_{i, n}\right|^{2}\right]\right\}=\min \left\{\mathbf{E}\left[\left|\mathbf{w}^{H} \mathbf{x}_{i, n}\right|^{2}\right]\right\}=\min \left\{\mathbf{w}^{H} \mathbf{E}\left[\mathbf{x}_{i, n} \mathbf{x}_{i, n}^{H}\right] \mathbf{w}\right\}=\min \left\{\mathbf{w}^{H} \mathbf{R}_{i, n} \mathbf{w}\right\}
$$

Equation 2.13.1

The criteria in Equation 2.13.1 are the general building blocks of all STAP derived algorithms. If we can apply a MVDR constraint

$$
\mathbf{w}^{H} \mathbf{S}=1
$$

Equation 2.14 
on Equation 2.13.1, then our constrained minimum variance distortion-less response can be achieved if we calculate the MVDR weight vector by

$$
\mathbf{w}^{\text {MVDR }}=\frac{\arg \min }{\mathbf{w}^{H} \mathbf{S}=1}\left\{\mathbf{w}^{H} \mathbf{R}_{i, n} \mathbf{w}\right\}
$$

Equation 2.15

where $\mathbf{s}=\frac{\mathbf{v}_{\theta}}{\left\|\mathbf{v}_{\theta}\right\|}$ is the desired steering vector and $\|x\|$ is the 2-norm operator. This weight vector $\mathbf{w}^{\text {MVDR }}$ can accomplish the two stated objectives.

Calculating the weight vector requires a method known as the Lagrange multiplier. This method can find the maximum or minimum of a function given constraints.

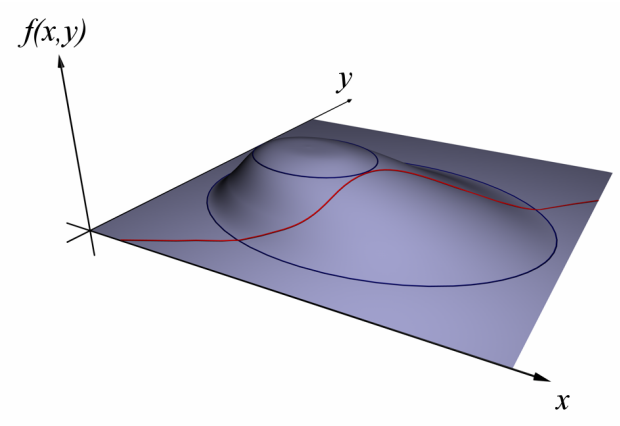

Figure 12: Function with constraint

Figure 12 shows a 3D function and its linear constraint. The multiplier can reform the function with new limitations set by the constraint. In that regard, our function transforms to

$$
L=\mathbf{w}^{H} \mathbf{R}_{i, n} \mathbf{w}+\lambda\left(\mathbf{w}^{H} \mathbf{s}-1\right)+\lambda^{*}\left(\mathbf{s}^{H} \mathbf{w}-1\right)
$$

Equation 2.16 
If we take gradient of $L$ with respect to $\mathbf{w}$ and set it equal to zero we

have

$$
\nabla_{w^{H}} L=\mathbf{R}_{i, n} \mathbf{w}+\lambda \mathbf{s}=0
$$

Equation 2.17

Our desired weight vector can be calculated with the following equation

$$
\mathbf{w}=-\lambda \mathbf{R}_{i, n}^{-1} \mathbf{s}
$$

Equation 2.18

Inserting the linear constraint of $\mathbf{w}^{H} \mathbf{s}=1$ and solve for the Langrage multiplier we would receive

$$
\lambda=-\left(\frac{1}{\mathbf{s}^{H} \mathbf{R}_{i, n}^{-1} \mathbf{s}}\right)
$$

Equation 2.19

Substitute above into Equation 2.18 yields the weight vector for optimal beam forming

$$
\mathbf{w}^{M V D R}=\mathbf{R}_{i, n}^{-1}\left(\frac{\mathbf{s}}{\mathbf{s}^{H} \mathbf{R}_{i, n}^{-1} \mathbf{s}}\right)
$$

Equation 2.20

Please note that $\mathbf{s}^{H} \mathbf{R}_{i, n}^{-1} \mathbf{s}$ from above is a normalizing factor and generally Equation 2.21 is expressed in its most general form

$$
\mathbf{w}^{M V D R}=\mathbf{R}_{i, n}^{-1} \mathbf{S}
$$

Equation 2.21 


\subsubsection{Adaptive beam-forming}

Optimal beam forming sounds really good in theory, unfortunately it sounds only good in theory. One important fact we must face in practice is that we do not have the interference covariance matrix or $\mathbf{R}_{i, n}$ which would require infinite number of samples. However, we can estimate it.

A sampled interference covariance matrix is

$$
\hat{\mathbf{R}}_{i, n}=\frac{1}{K} \sum_{k=1}^{K}\left[\mathbf{x}_{i, n}(k) \mathbf{x}_{i, n}^{H}(k)\right]
$$

Equation 2.22

$K$ is the total number of training samples available and $\mathbf{x}_{i, n}(k)$ is the $k$ th

training sample. The sampled covariance matrix $\hat{\mathbf{R}}_{i, n}$ is the maximum likelihood estimate of the true covariance matrix $\mathbf{R}_{i, n}$ (70). With a sampled covariance matrix, our weight vector equation updates to

$$
\mathbf{W}^{M V D R, S M I}=\hat{\mathbf{R}}_{i, n}^{-1}\left(\frac{\mathbf{S}}{\mathbf{S}^{H} \hat{\mathbf{R}}_{i, n}^{-1} \mathbf{S}}\right)
$$

Equation 2.23

Likewise its most general form is

$$
\begin{gathered}
\mathbf{W}^{M V D R, S M I}=\hat{\mathbf{R}}_{i, n}^{-1} \mathbf{S} \\
\text { Equation } \mathbf{2 . 2 4}
\end{gathered}
$$


The tag SMI stands for sample matrix inverse. Though this Equation 2.24 is practical, there are unfortunate consequences. For example, before the STAP system can be used for target detection, it must first be trained with data samples of the environment. These training samples must be "target free" and plentiful, both luxuries in practice.

There are two basic types of training data: "target free" training data, and "target in" training data. The "target free" training data are such that $\mathbf{x}_{i, n}=\mathbf{x}_{n}+\mathbf{x}_{j}$ and "target in" data is $\mathbf{x}_{s, i, n}=\mathbf{x}_{s}+\mathbf{x}_{n}+\mathbf{x}_{j}$. The covariance matrix that we wish to estimate is the interference plus noise covariance $\mathbf{R}_{i, n}$. In some applications like radar, "target free" training data is always available and will be considered plentiful in our simulations. Since radar can take measurements at ranges shorter and longer than the target, $\mathbf{x}_{i, n}$ is more easily obtained. However for applications such as passive sonar, it can only collect "target in" training data because it cannot do ranging. Applications such as sonar will have to depend on "target in" training data $\mathbf{x}_{s, i, n}$ and deal with its performance degradation effect as best as possible. Fortunately in this thesis we only deal with airborne radar system and we can assume that "target free" training data is always available. 
Our second concern is the number of training samples. Typically, the more samples acquired the better, however in practice that is not a luxury to count on. With battlefields becoming more complex and adversaries becoming more sophisticated, good "target free" training samples are difficult to acquire thus spawning interest to develop systems that can perform well in "sample starved" environments. A common rule of thumb is $K \approx 2 \mathbf{N}$ samples are needed for good performance, or to be within $3 \mathrm{~dB}$ of the optimum. However, it has been shown that MWF can achieve competitive performance with only $K \approx 1 \mathbf{N}$ samples.

Quality of training data is also a concern. The training data samples $\mathbf{x}_{i, n}$ are needed to estimate the interference covariance matrix $\mathbf{R}_{i, n}$. These samples are also realized from this covariance matrix. Hence $\mathbf{x}_{i, n}$ are formed from independent and identically distributed (IID) snapshots of $\mathbf{R}_{i, n}$. Generally, training samples are taken either from "target free" spatial locations or collected across time. In the first option, samples taken from different physical locations rely on an assumption of spatial homogeneity. For the second option, samples taken across time rely on the assumption of stationary interference (i.e. no moving jammers). In practice, our ability to acquire good IID samples that can satisfy either of these assumptions is severely limited. Hence algorithms like MWF that 
can operate in sample starved environments draw strong practical interest.

\subsubsection{Performance metrics}

Here we detail two important performance metrics: the signal to interference plus noise ratio (SINR) and mean square error (MSE). In contrast to the previous described metrics SNR and JNR that describes interferences at element level; SINR and MSE describes performance at array level, more precisely the output of the array beam former.

In this section we take a closer look at these performance metrics. We will derive their expressions, show their relationship to each other and evaluate them. Simulations of these metrics will be given in later chapters to clarify their behavior in complex environments. These evaluations will demonstrates the advantage of the MWF, particularly in environments with jamming, clutter and low sample support.

For the remainder of this thesis, this author assumes that "target free" training data $\mathbf{x}_{i, n}$ is available, that there is no steering vector mismatch (i.e., the signal arrives from a known direction and the element positions are known), and that the distortion-less constraint is relative to the unit-norm steering vector (i.e. $\mathbf{w}^{H} \mathbf{s}=1$ where $\mathbf{s}^{H} \mathbf{s}=1$ ).

The SINR is similar to the SNR but also count the effect of jamming. Maximizing SINR is a criterion in most detection and estimation problems 
(48). Radar detection performance is directly related to SINR. For example, with assumptions of Gaussian interference and known statistics, it can be shown that maximizing the SINR leads to maximizing the probability of detection (9). SINR can be computed at both the input (i.e., element level) and output of the array (i.e., beam former). This thesis is mostly concerned with SINR at array output.

The mean square error (MSE) is a metric of an adaptive filter's ability to cancel out interference. MSE is the most fundamental performance metrics. SINR is inversely proportional to MSE.

MSE for "target free" training is

$$
M S E=\mathbf{E}\left[\left|\mathbf{w}^{H} \mathbf{x}_{i, n}\right|^{2}\right]=\mathbf{w}^{H} \mathbf{R}_{i, n} \mathbf{w}
$$

\section{Equation 2.25}

where $\mathbf{R}_{i, n}$ is the known interference-plus-noise covariance matrix and $\mathbf{w}$ is the calculated weight vector from acquired samples.

The output SINR is the ratio of the signal power to the interferenceplus-noise power at the output of the array

$$
\operatorname{SINR}=\frac{\left|\mathbf{w}^{H} \mathbf{x}_{s}\right|^{2}}{E\left[\left|\mathbf{w}^{H} \mathbf{x}_{i, n}\right|^{2}\right]}=\frac{\sigma_{s}^{2}\left|\mathbf{w}^{H} \mathbf{v}_{\theta}\right|^{2}}{\mathbf{w}^{H} \mathbf{R}_{i, n} \mathbf{w}}
$$

Equation 2.26

$\mathbf{v}_{\theta}$ is the spatial array manifold vector, recall that $\mathbf{s}=\frac{\mathbf{v}_{\theta}}{\left\|\mathbf{v}_{\theta}\right\|}$ which implies

that $\mathbf{v}_{\theta}=\sqrt{\mathbf{N}} \mathbf{s}$. Substituting this into Equation 2.26 yields 


$$
\operatorname{SINR}=\frac{N \sigma_{s}^{2}\left|\mathbf{w}^{H} \mathbf{s}\right|^{2}}{\mathbf{w}^{H} \mathbf{R}_{i, n} \mathbf{w}}
$$

Equation 2.27

Assuming distortion-less response $\mathbf{w}^{H} \mathbf{s}=1$, Equation 2.27 is reduced to

$$
\operatorname{SINR}=\frac{N \sigma_{s}^{2}}{\mathbf{w}^{H} \mathbf{R}_{i, n} \mathbf{w}}=\frac{N \sigma_{s}^{2}}{M S E}
$$

Equation 2.28

Therefore we conclude that our output SINR is inversely proportional to MSE.

\subsection{Space-time adaptive processing}

We are now ready to thoroughly discuss STAP. Before we get down into the equations there are few things we should keep in mind. First, recall in spatial array processing (SAP) our total degree of freedom (DoF) equals the number of elements because we can apply a complex weight to each receiver as shown in Figure 13. These weights give us a certain degree of control over the array output.

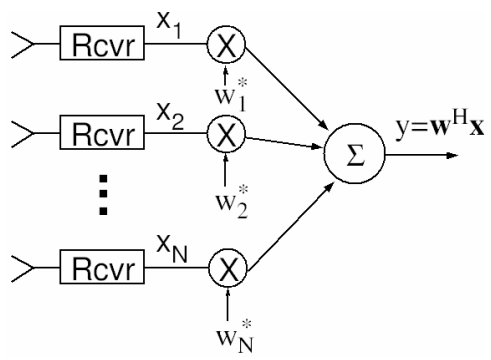

Figure 13: Receiver array with weight 
For STAP, our transmitter emits a train of M pulses. Our receiver receives the echoes and can apply a different complex weight to each pulse return, therefore in STAP, our degree of freedom equals the total number of receiving elements $(N)$ multiply the total number of pulses in the pulse-train $(M)$ which equals to NxM. Another important point we should consider is the progressive phase shift from these successive pulses. Like the previously discussed spatial array manifold vector which account for shift due to spatial displacement, another progressive shift due to temporal factors must be accounted, this requires us to add the temporal array manifold vector. We will discuss this temporal vector later in this section. The third and final note is the received data format. In STAP, the received data have the dimension of NxMxR or cubic as shown in Figure 14.

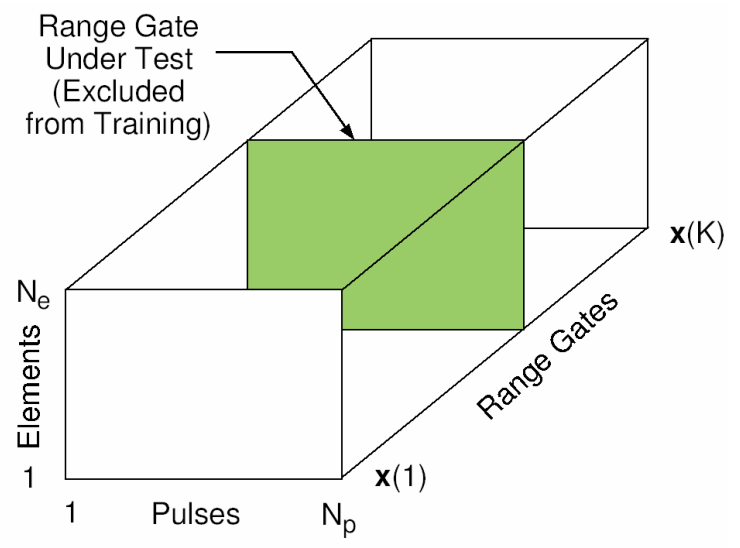

Figure 14: Data cube 
The dimension of $\mathrm{R}$ in this data cube corresponds to the number of range gates. Range gates are Doppler filters that can filter signal returns base on their Doppler shift due to range. Typically the data from the range gate under test is excluded from the training samples to enforce condition of "target free" training. Usually nearby range gates are also excluded as guard cells to excluded target side lobe energy from creeping in.

With these points given, we can now get into STAP. Recall that in SAP we used a spatial array manifold vector $\mathbf{v}_{\theta}$ to take account of spatial phase shift from inter-element displacement. In STAP we must also account the inter-pulse progressive phase shift as well. Hence we show the temporal array manifold vector,

$$
\mathbf{v}_{w}=\left[e^{j 0} e^{j 2 \pi w_{n}} \ldots e^{j 2 \pi(M-1) w_{n}}\right]^{T}
$$

Equation 2.29

In this equation, $w_{n}=\frac{f}{f_{p}}$ is the normalized Doppler frequency, $f$ is the Doppler of the signal and $f_{p}$ is the pulse repetition frequency (PRF). Dimension of temporal array manifold vector is $\mathbf{M x} \mathbf{1}$ where $\mathbf{M}$ is number of pulses. 
Since we are dealing with space-time adaptive processing, our steering vector must now account for both spatial and temporal phase shifts. Therefore our space-time steering vector is

$$
\mathbf{S}=\mathbf{v}_{s t}=\mathbf{v}_{w} \otimes \mathbf{v}_{\theta}
$$

Equation 2.30

where $\otimes$ is the Kronecker product. The Kronecker product of an AxB matrix and a CxD matrix result in a matrix with dimension of $\mathrm{ACXBD}$.

With the space-time steering vector s, our weight vector

$\mathbf{w}^{M V D R, S M I}=\hat{\mathbf{R}}_{i, n}^{-1} \mathbf{S}$, becomes a 2D space-time filter that filters received signals based on both the spatial steering vector and the temporal steering vector.

An added dimension of filtering comes with added dimensions of complexity. One of these additional complexities is the interference: clutter. The term clutter generally covers all unwanted echoes from the natural environment. We restrict our attention to land clutter only, which has zero inherent velocity. High reflectivity factors such as buildings and ground mineral deposits (a.k.a. discrete) and low reflectivity factors from plain ground falls into this category. This clutter interference, like jamming and noise, must also be suppressed for effective target detection. 


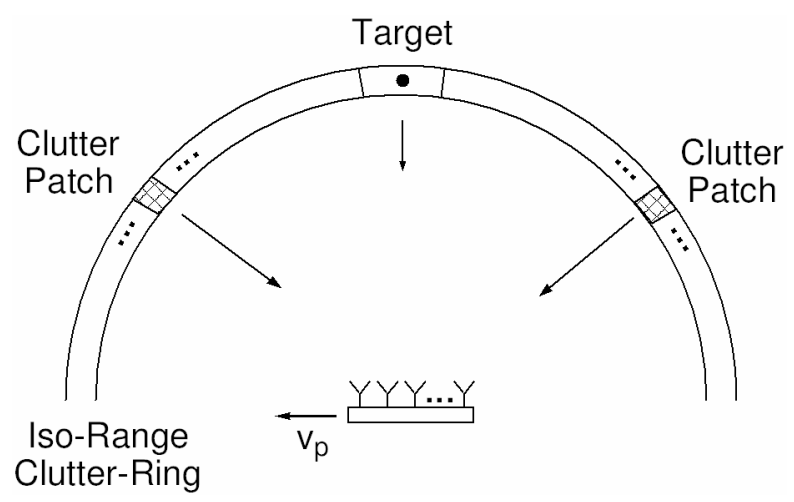

Figure 15: Clutter ring

\subsubsection{Clutter}

For airborne mounted radar that moves with velocity $v_{p}$, received ground clutter is viewed as a "clutter ring" with the same radius as the desired target signal. Figure 15 shows an example. For simplicity sake we only model half the ring by assuming our array back lobes are heavily tapered. The width of this ring is also the range resolution of the radar

$$
\Delta R=c / 2 B
$$

Equation 2.31

where $c$ is speed of light and $B$ is the array operating bandwidth. For our simulations we assume 180 clutter patches in this ring. Echo from each patch contains a Doppler shift due to radar platform velocity which can be calculated by

$$
f_{c}=\frac{2 v_{p}}{\lambda} \sin \theta_{c}
$$

Equation 2.32

$\theta_{c}$ is the angle of clutter patch. 


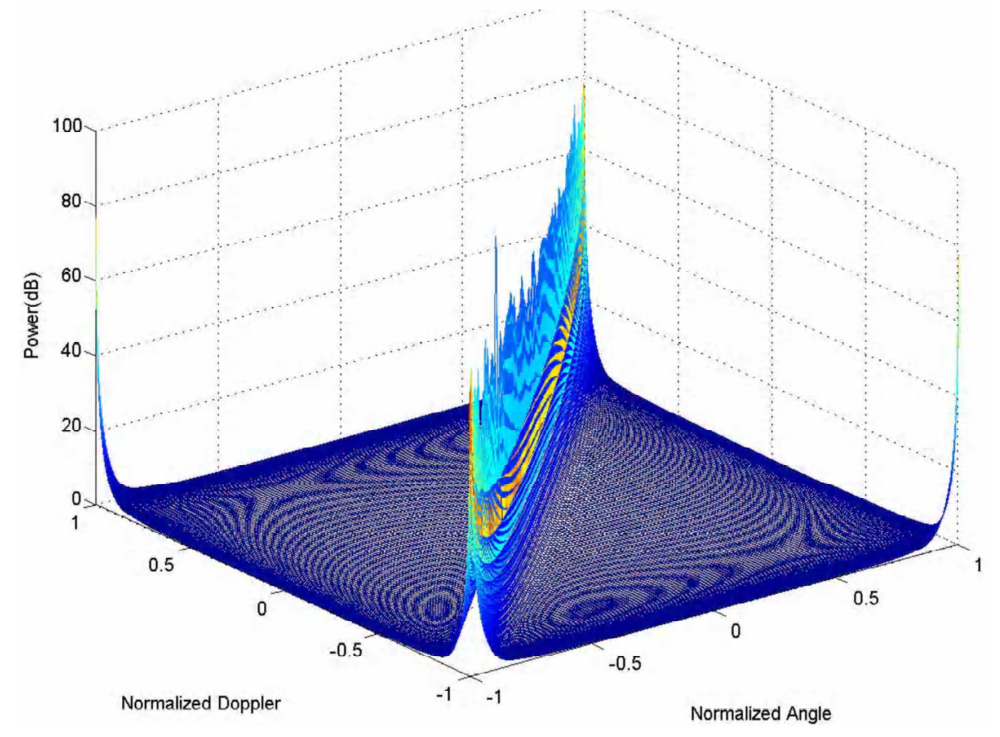

Figure 16: Clutter "ridge"

Figure 16 illustrates the resulting "clutter ridge" seen by radars in the normalized angle-Doppler domain. This "ridge" is the result of Doppler shift of received clutter due to platform velocity and hence is a linear function of the spatial frequency. The slope of this clutter ridge for an array with half-wavelength spacing is

$$
\beta=\frac{4 v_{p}}{\lambda f_{r}}
$$

Equation 2.33

where $f_{r}$ is the radar PRF. The term $\beta$ dictates the number of times that the clutter Doppler spectrum aliases into the unambiguous Doppler space (63). Figure 16 corresponds to $\beta=1$ (a.k.a. Displaced Phase Center Array 
mode) where the Doppler space is fully populated but there is no aliasing.

Figure 17 illustrates this ridge behavior for other values of $\beta$.
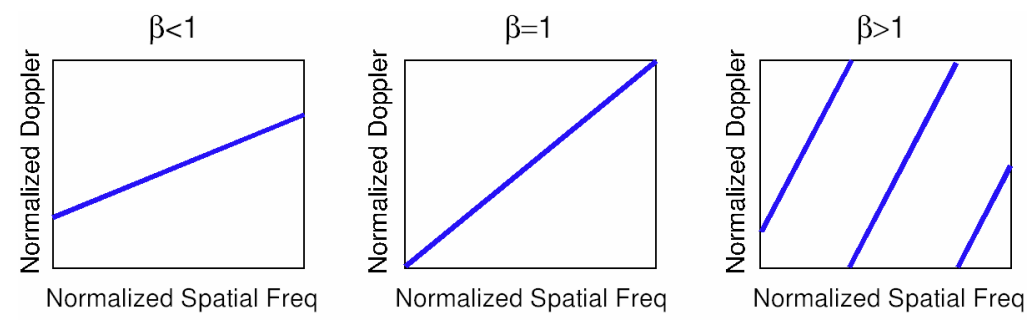

Figure 17: Top view of clutter ridges of various $\beta$ values

For simplicity sake, we assume $\beta=1$ throughout this thesis. The total clutter power received by our array is the sum of clutter power from each clutter patch, with each patch modeled as a random Gaussian process. Hence our space-time clutter covariance matrix is

$$
\mathbf{R}_{c}=\sum_{i=1}^{N_{r}} \sum_{j=1}^{N_{C}} \sigma_{C}^{2}(i, j)\left[\mathbf{v}_{w_{c}}(i, j) \mathbf{v}_{w_{c}}(i, j)^{H}\right] \otimes\left[\mathbf{v}_{\theta_{c}}(i, j) \mathbf{v}_{\theta_{c}}(i, j)^{H}\right]
$$

Equation 2.34

where $N_{c}$ is the number of evenly distributed clutter patches in azimuth, $N_{r}$ is the number of range ambiguities, $\sigma_{C}^{2}(i, j)$ corresponds to received clutter power from the $i j^{\text {th }}$ patch, $\mathbf{v}_{w_{c}}$ and $\mathbf{v}_{\theta_{c}}$ are the temporal and spatial array manifold vectors associated with the $i j^{\text {th }}$ clutter patch, and $\otimes$ is again the Kronecker product.

The total clutter to noise ratio would be 


$$
C N R=\frac{\sum_{i=1}^{N_{r}} \sum_{j=1}^{N_{C}} \sigma_{C}^{2}(i, j)}{\sigma_{n}^{2}}
$$

Equation 2.35

where $\sigma_{n}^{2}$ is the element level received noise power.

The desired signal, noise, and jamming models discussed earlier for SAP must be extended for STAP. We use our new space-time array manifold vector

$$
\mathbf{S}=\mathbf{v}_{s t}=\mathbf{v}_{w} \otimes \mathbf{v}_{\theta}
$$

Equation 2.36

to accomplish this. For example, the desired signal model for STAP would be

$$
\mathbf{x}_{s}=x \mathbf{v}_{s t}=x \mathbf{S}
$$

Equation 2.37

where $x$ is again the complex baseband signal received at leftmost element for the first pulse and $\mathbf{v}_{s t}$ is the space-time array manifold vector.

The noise model in STAP changes little from its SAP origin and is

$$
\mathbf{R}_{n}=\sigma_{n}^{2}\left(\mathbf{I}_{N} \otimes \mathbf{I}_{M}\right)
$$

Equation 2.38

where $\sigma_{n}^{2}$ is the thermal noise power at the $n^{\text {th }}$ receiver, $\mathbf{I}_{N}$ and $\mathbf{I}_{M}$ are identity matrices of dimension $\mathbf{N x N}$ and $\mathbf{M x M}$ respectively. The element 
level thermal noise is still modeled as a Gaussian random process because these noises are spatially and temporally uncorrelated.

Unfortunately jamming is spatially correlated but uncorrelated pulse to pulse. Therefore the jamming covariance matrix for a single jammer can be expressed as (63)

$$
\mathbf{R}_{j}=\sigma_{j}^{2}\left[\mathbf{I}_{M} \otimes\left[\mathbf{v}_{\theta_{j}} \mathbf{v}_{\theta_{j}}^{H}\right]\right]
$$

Equation 2.39

where $\sigma_{j}^{2}$ is the jammer power, $\mathbf{v}_{\theta_{j}}$ is this jammer's spatial array manifold vector.

These undesired signals (noise, clutter, and jamming) for STAP are all assumed to be mutually uncorrelated. Therefore, the total interference plus noise covariance matrix $\mathbf{R}_{i, n}$ for STAP can be expressed as the sum of the individual covariance matrices

$$
\mathbf{R}_{i, n}=\mathbf{R}_{n}+\mathbf{R}_{c}+\sum_{i=1}^{N_{j}} \mathbf{R}_{j}(i)
$$

Equation 2.40 


\section{Chapter 3}

\section{Reduce rank processing}

In this chapter I will describe several algorithms for reduced rank

processing. My focus is on statistical approaches such as multi-stage

Wiener filter (MWF), principal component (PC) and to a less extent cross

spectral metric (CSM). All of these algorithms apply to the partially

adaptive arrays discussed in Chapter 2. I will detail all three but the MWF

is the focus in this thesis. Another rank reducing technique called

diagonal loading (DL) will be discussed as well. I will also describe the

relationships among these reduce rank techniques for clarity purposes. 
The general role of a rank reducing transformation (RRT) is to convert the input data $\mathbf{x}$ from a full rank, $\mathbf{N}$ dimensional vector, to the reduced rank, $r$ dimensional vector $\mathbf{z}$, where $r$ is less than $\mathbf{N}$.

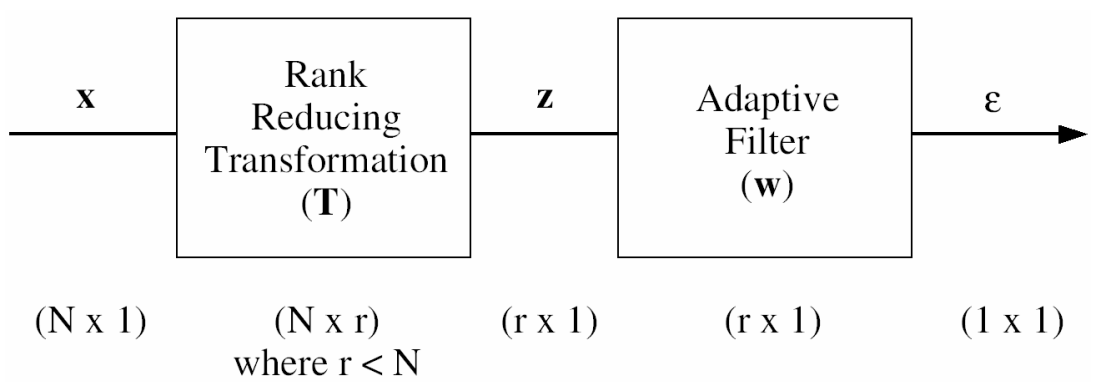

Figure 18: Reduce rank transformation

The adaptive filter then operates on the reduced dimensional space for desired results. Generally there are two categories for rank reducing transformation: deterministic transforms and statistically optimized transforms (35). Example of the first type is beam space processing and examples of latter include principal components $(7,69$, $37)$, the cross-spectral metric $(1,73)$, and the multistage Wiener filter $(16)$. This thesis primarily focuses on the MWF but we also discuss principal components to use it as a benchmark due to its popularity.

The deterministic and statistical approaches I mentioned thus far are also known as "hard stop" techniques because they physically truncate the data to a reduced dimensional space. There is an alternative, also known as "soft stop" technique. One example is diagonal loading $(4,11)$. Diagonal loading doesn't reduce the physical dimensions of computational space but does reduce the required 
number of adaptive degrees of freedoms needed to process. I will discuss more on DL in later sections. Finally, "soft stop" techniques such as DL can work in conjunction with "hard stop" techniques such as MWF to improve performance.

\subsection{Principal Components}

The concept of Eigen-space beam former has been studied by many authors for decades $(69,30,51,5,8,37,15)$. A popular variant is the principal components (PC) $(69,37)$. PC uses the Eigen-value decomposition (EVD) to produce a low rank estimate of the sampled

covariance matrix $\hat{\mathbf{R}}_{\mathbf{x}}$. The selling point of this technique is that this lower rank estimate would still be a good approximation to the original but it would dramatically reduce the required computer processing power. This "speed for accuracy" trade off is widely accepted in the industry due to benefit of reduced cost. Consider the MVDR-SMI beam former we discussed earlier

$$
\mathbf{W}^{M V D R, S M I}=\hat{\mathbf{R}}_{\mathbf{x}}^{-1}\left(\frac{\mathbf{s}}{\mathbf{s}^{H} \hat{\mathbf{R}}_{\mathbf{x}}^{-1} \mathbf{S}}\right)
$$

Equation 3.1

where 


$$
\hat{\mathbf{R}}_{\mathbf{x}}=\frac{1}{K} \sum_{k=1}^{K}\left[\mathbf{x}_{i, n}(k) \mathbf{x}_{i, n}^{H}(k)\right]
$$

Equation 3.2

and $K$ is the number of training snapshots. An EVD of $\hat{\mathbf{R}}_{\mathbf{x}}$ would be

$$
\hat{\mathbf{R}}_{\mathbf{x}}=\sum_{i=1}^{N} \lambda_{i} \mathbf{v}_{i} \mathbf{v}_{i}^{H}
$$

\section{Equation 3.3}

where $\lambda_{i}$ and $\mathbf{v}_{i}$ represent the ith eigenvalue and eigenvector of $\hat{\mathbf{R}}_{\mathbf{x}}$ and $N$ is the total number of degree of freedoms.

The best reduced $r$ rank approximation of $\hat{\mathbf{R}}_{\mathbf{x}}$ is formed by retaining the $r$ largest eigenvalues and their corresponding eigenvectors and eliminate the rest. Therefore

$$
\hat{\mathbf{R}}_{\mathbf{x}}{ }^{(P C)}=\sum_{i=1}^{r_{P C}} \lambda_{i} \mathbf{v}_{i} \mathbf{v}_{i}^{H}
$$

Equation 3.4

where $r_{P C}$ is less than $N$ but contains the principal components of $\hat{\mathbf{R}}_{\mathbf{x}}$ or

the components with most signal power. Selecting the value for $r_{P C}$ is

completely arbitrary. A typical strategy to select $r_{P C}$ is to find the number of eigenvalues that are above the noise floor. One assumption from Equation 3.4 is that the eigenvalues are ordered from highest to lowest. Meaning that the highest eigenvalue is at $i=1$, the next highest eigenvalue is at $i=2$, and so forth. 
Figure 19 below is a block diagram example of PC-SI system.

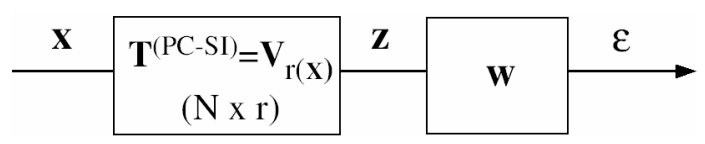

Figure 19: Principal component signal independent

Principal component signal independent (PC-SI) algorithm is a data dependent signal independent rank reducing algorithm. It is considered to be data dependent because the data $\mathbf{X}$ is considered in weight vector calculation. It is considered signal independent because the steering vector $\mathbf{s}$ is not. One advantage of this algorithm is that it is simple. A disadvantage is that we lose performance by not taking s into account. Whether this is applicable really depends on the application, error tolerance and cost. The PC-SI weight vector would be

$$
\mathbf{w}^{M V D R, S M I, P C-S I}=\left(\hat{\mathbf{R}}_{\mathbf{x}}^{P C}\right)^{-1}\left(\frac{\mathbf{s}}{\mathbf{s}^{H}\left(\hat{\mathbf{R}}_{\mathbf{x}}^{P C}\right)^{-1} \mathbf{S}}\right)
$$

Equation 3.5

Another PC variation is PC-SD, SD as in signal dependent. Figure 20 shows a block diagram example.

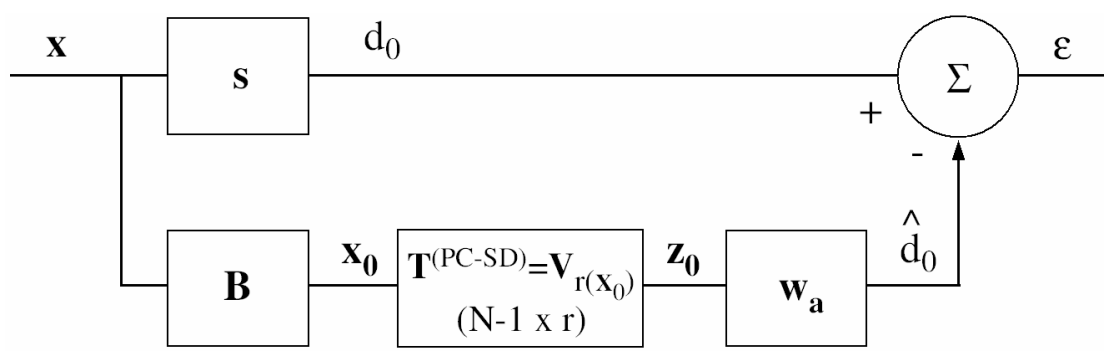

Figure 20: Principal component signal dependent 
As the name suggest, PC-SD does take the steering vector s (or main lobe response) into account for rank reduction. The block $\mathbf{B}$ is a set of vectors that are orthonormal to s (i.e. side lobe responses). The steering vector for PC-SD would be

$$
\mathbf{s}_{P C-S D}=\mathbf{s}-\mathbf{B} \mathbf{w}_{a}
$$

Equation 3.6

where $\mathbf{w}_{a}$ is

$$
\mathbf{w}_{a}=\left(\hat{\mathbf{R}}_{\mathbf{x}}^{P C}\right)^{-1} \mathbf{r}_{\mathbf{z}_{0} \mathbf{d}_{0}}
$$

Equation 3.7

and $\mathbf{r}_{\mathbf{z}_{0} \mathbf{d}_{0}}$ is the cross correlation vector between $\mathbf{z}_{0}$ and $\mathbf{d}_{0}$. The spacetime weight vector would be

$$
\mathbf{W}^{M V D R, S M I, P C-S D}=\left(\hat{\mathbf{R}}_{\mathbf{x}}^{P C}\right)^{-1}\left(\frac{\mathbf{s}_{P C-S D}}{\mathbf{S}_{P C-S D}{ }^{H}\left(\hat{\mathbf{R}}_{\mathbf{x}}^{P C}\right)^{-1} \mathbf{s}_{P C-S D}}\right)
$$

Equation 3.8

\subsection{Cross spectral metric}

In this section we briefly describe the cross-spectral metric (CSM) algorithm for rank 
reduction $(1,73)$. We will not discuss CSM too deeply because we need to focus on PC and MWF, however skimming this can provide clarity for other techniques.

Like the PC, CSM also uses an eigenvector basis for the rank reducing transformation. However, CSM selects those eigenvectors base on maximizing the signal to interference plus noise ratio (SINR) instead of high eigenvalues. This is explained as follows (35), recall that

$$
\operatorname{SINR}=\frac{N \sigma_{s}^{2}}{\mathbf{w}^{H} \mathbf{R}_{i, n} \mathbf{w}}
$$

Equation 3.9

where $N$ is the number of adaptive degrees-of-freedom, $\sigma_{s}^{2}$ is the element-level signal power, $\mathbf{w}$ is the weight vector of interest, and $\mathbf{R}_{i, n}$ is the interference plus noise covariance matrix.

Next, consider the optimal MVDR weight vector previously discussed

$$
\mathbf{w}^{M V D R}=\mathbf{R}_{i, n}^{-1}\left(\frac{\mathbf{s}}{\mathbf{s}^{H} \mathbf{R}_{i, n}^{-1} \mathbf{s}}\right)
$$

Equation 3.10

and substitute this into Equation 3.9 results to

$$
\operatorname{SINR}=N \sigma_{s}^{2}\left(\mathbf{s}^{H} \mathbf{R}_{i, n}^{-1} \mathbf{S}\right)
$$

Equation 3.11 
Now if we perform an eigenvalue decomposition (EVD) of the covariance matrix, $\mathbf{R}_{i, n}=\mathbf{V} \Lambda \mathbf{V}^{H}$, where $\mathbf{V}$ is the eigenvector matrix and $\Lambda$ is the diagonal matrix of eigenvalues. The SINR can be transformed to

$$
\operatorname{SINR}=N \sigma_{s}^{2}\left(\mathbf{s}^{H} \mathbf{V} \Lambda^{-1} \mathbf{V}^{H} \mathbf{s}\right)=N \sigma_{s}^{2}\left(\sum_{i=1}^{N} \frac{\left|\mathbf{v}_{i}{ }_{i}^{H} \mathbf{s}\right|^{2}}{\lambda_{i}}\right)
$$

Equation 3.12

Therefore CSM algorithm retains eigenvectors that maximize the

metric $\frac{\left|\mathbf{v}_{i}{ }^{H} \mathbf{s}\right|^{2}}{\lambda_{i}}$. These eigenvectors will be different from the eigenvectors chose by PC therefore the resulting weight vector is different as well. Performance wise, CSM is generally considered superior to PC but lower than MWF. Aside from these we will not discuss CSM in any more detail.

\subsection{Multi-stage Wiener filter}

Rank reduction for Wiener filter is heavily dependent on the cross correlation vector as shown previously

$$
\mathbf{w}_{\text {opt }}=\mathbf{R}_{x_{0}}^{-1} \mathbf{r}_{x_{0} d_{0}}
$$

Equation 3.13

where the weight vector is a function of both covariance matrix $\mathbf{R}_{x_{0}}$ and cross correlation vector $\mathbf{r}_{x_{0} d_{0}}$. Derived from the original Wiener filter, the multi-stage Wiener filter was introduced in (16). Its constrained form structure is shown in Figure 21. 


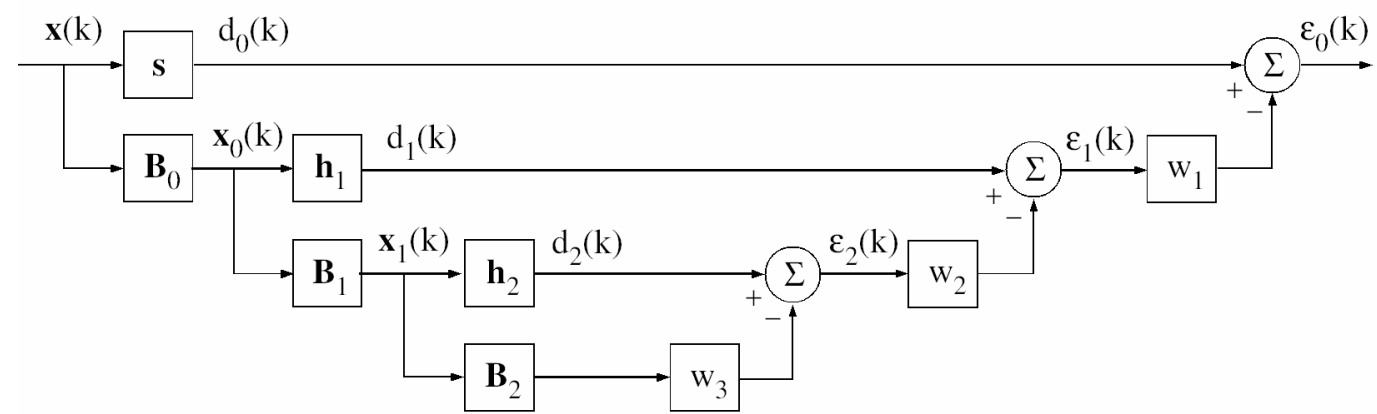

Figure 21: A three stage adaptive filter

In forward recursion, the filter decomposes the sampled data snapshot $\mathbf{x}$ with a sequence of orthogonal projection blocks like $\mathbf{B}_{0}(16)$. Rank reduction can be accomplished by truncating these decomposition stages to a desired number $\mathbf{r}^{m w f}$. The result is a reduced rank transformation basis that spans the Krylov subspace instead of the eigenvector basis like PC and CSM $(54,13)$.

$$
K\left(\mathbf{s}, \mathbf{R}_{\mathbf{x}}, \mathbf{r}^{m w f}\right)=\underset{\text { Equation } 3.13}{\operatorname{span}\left\{\mathbf{s}, \mathbf{R}_{\mathbf{x}} \mathbf{s}, \mathbf{R}_{\mathbf{x}}^{2} \mathbf{S}, \ldots, \mathbf{R}_{\mathbf{x}}^{\left(\mathbf{r}^{m w f}-1\right)} \mathbf{s}\right\}}
$$

Since it tailors its basis selection to the desired steering vector $\mathbf{s}$, the MWF is able to operate in a more compact subspace than PC and CSM (35).

After the forward recursion is completed, the MWF computes a series of scalar weights $\left(w_{1}, w_{2}\right.$, etc $)$ at each stage and subsequently combine them to form the overall MWF weight vector

$$
\mathbf{w}^{m w f}=\mathbf{s}-w_{1} \mathbf{B}_{0}^{H} \mathbf{h}_{1}+w_{1} w_{2} \mathbf{B}_{0}^{H} \mathbf{B}_{1}^{H} \mathbf{h}_{2}-w_{1} w_{2} w_{3} \mathbf{B}_{0}^{H} \mathbf{B}_{1}^{H} \mathbf{B}_{2}^{H} \mathbf{h}_{3}+\ldots
$$

Equation 3.14 
This technique has many desirable properties. First, its main computation operation is the simple vector cross correlation. Second, it does not form a covariance matrix which requires substantial computation workload (59). Last, it doesn't need matrix inversion or eigenvector decomposition, both of which are expensive operations ( 13 , 70).

\subsection{Diagonal loading}

Diagonal loading is very common beamforming technique that provides a variety of benefits. For example, it can acquire beamforming solution in situations where samples are less than the degrees of freedoms. It can also add robustness to beamformers when dealing with mismatch problems from direction of arrival, element position error, gain/phase perturbations and statistical mismatch from finite sample support $(4,70)$.

Diagonal loading can also reduce rank. It is categorized as a "soft stop". Unlike the previously discussed "hard" stop algorithms like PC, CSM and MWF which forcefully reduces rank, diagonal loading gently "turn off" the eigenvectors that have small eigenvalues and therefore reduces the number of adaptive degrees of freedom.

A demonstration is as follows. Consider first the beamformer weight vector with diagonal loading 


$$
\mathbf{W}^{d l}=\tilde{\mathbf{R}}_{\mathbf{x}}^{-1}\left(\frac{\mathbf{S}}{\mathbf{S}^{H} \tilde{\mathbf{R}}_{\mathbf{x}}^{-1} \mathbf{S}}\right)
$$

Equation 3.15

where

$$
\begin{gathered}
\tilde{\mathbf{R}}_{\mathbf{x}}=\hat{\mathbf{R}}_{\mathbf{x}}+\sigma_{L}^{2} \mathbf{I} \\
\text { Equation 3.16 }
\end{gathered}
$$

$\hat{\mathbf{R}}_{\mathbf{x}}$ is the sample covariance matrix and $\sigma_{L}^{2}$ is the loading value. One would immediately recognize from Equation 3.16 that a mere constant value of $\sigma_{L}^{2}$ is added to the diagonal elements of $\hat{\mathbf{R}}_{\mathbf{x}}$. Why is this important? Consider that

$$
\hat{\mathbf{R}}_{\mathbf{x}}=\frac{1}{K} \sum_{k=1}^{K}\left[\mathbf{x}(k) \mathbf{x}^{H}(k)\right]=\frac{1}{K} \mathbf{X} \mathbf{X}^{H}=\mathbf{V} \Lambda \mathbf{V}^{H}
$$

Equation 3.17

where $\Lambda$ is a diagonal matrix of eigenvalues and $\mathbf{V}$ is the corresponding eigenvector matrix. So then

$$
\tilde{\mathbf{R}}_{\mathbf{x}}=\frac{1}{K} \mathbf{X} \mathbf{X}^{H}+\sigma_{L}^{2} \mathbf{I}=\frac{1}{K}\left(\mathbf{X} \mathbf{X}^{H}+\beta^{2} \mathbf{I}\right)
$$

Equation 3.18

where $\beta^{2}=\sigma_{L}^{2} K$. The eigenvalue decomposition (EVD) of $\tilde{\mathbf{R}}_{\mathbf{x}}$ results to

$$
\left[\mathbf{X X}^{H}+\beta^{2} \mathbf{I}\right]=\mathbf{V} \tilde{\Lambda} \mathbf{V}^{H}
$$

Equation 3.19 
where $\tilde{\Lambda}=\Lambda+\beta^{2} \mathbf{I}$. From Equation 3.19, a constant value of $\beta^{2}$ is added to all eigenvalues of $\Lambda$, hence "boosting" them all by the same amount.

The inverse of $\tilde{\mathbf{R}}_{\mathbf{x}}$ can be manipulated in a similar fashion because

$$
\left(\mathbf{V} \tilde{\Lambda} \mathbf{V}^{H}\right)^{-1}=\mathbf{V} \tilde{\Lambda}^{-1} \mathbf{V}^{H}
$$

Equation 3.20

given that $\mathbf{V}$ is unitary, therefore $\mathbf{V}^{H}=\mathbf{V}^{-1}$.

The key in implementing diagonal loading $(D L)$ is to load $\hat{\mathbf{R}}_{\mathbf{x}}$ with the "right" level. Thus one can consider DL as weight factor that depends on the magnitude of the each eigenvalue. If the eigenvalues are too large relative to the diagonal loading level then the system performs as though no diagonal loading was applied and each eigenvector is retained for weight vector calculation. Conversely, if the eigenvalues are too small then all eigenvectors are essentially discarded from weight vector calculation (12). These two circumstances are generally labeled as under-loading and over-loading respectively. If the "right" loading level can be applied then we can effectively "turn on" eigenvectors that have important dynamics while "turn off" eigenvectors that does not. In practice, DL is typically chosen between 5 to $10 \mathrm{~dB}$ above the environment noise level. 


\subsection{Relationships between DL, PC and}

\section{MWF}

Before we move on to the next chapter we should understand a concept known as filter factors and how it connects DL, PC and MWF. Recall from the full rank Wiener filter

$$
\mathbf{W}_{a, o p t}^{f r}=\hat{\mathbf{R}}_{x_{0}}^{-1} \mathbf{r}_{x_{0} d_{0}}
$$

Equation 3.21

where $\hat{\mathbf{R}}_{x_{0}}$ can be decomposed using the EVD into

$$
\hat{\mathbf{R}}_{x_{0}}=\frac{1}{K}\left[\mathbf{X}_{0} \mathbf{X}_{0}^{H}\right]=\frac{1}{K}\left[\mathbf{V} \Lambda \mathbf{V}^{H}\right]=\frac{1}{K}\left[\sum_{i=1}^{N} \lambda_{i} \mathbf{v}_{i} \mathbf{v}_{i}^{H}\right]
$$

Equation 3.22

and

$$
\mathbf{r}_{x_{0} d_{0}}=\frac{1}{K}\left[\mathbf{X}_{0} \mathbf{d}_{0}^{H}\right]
$$

Equation 3.23

There is another decomposition we can use, singular value decomposition (SVD), where a data snapshot can be transformed to

$$
\mathbf{X}_{0}^{H}=\mathbf{U} \Sigma \mathbf{V}^{H}=\sum_{i=1}^{N} \mathbf{u}_{i} \sigma_{i} \mathbf{v}_{i}^{H}
$$

Equation 3.24

where $\sigma_{i}$ are the singular values, $\mathbf{u}_{i}$ and $\mathbf{v}_{i}$ are the left and right singular vectors. Combining Equation 3.22, 3.23 and 3.24 into 3.21 yields 


$$
\mathbf{w}_{a, o p t}^{f r}=\left[\mathbf{X}_{0} \mathbf{X}_{0}^{H}\right]^{-1}\left[\mathbf{X}_{0} \mathbf{d}_{0}^{H}\right]=\sum_{i=1}^{N} \frac{\mathbf{u}_{i}^{H} \mathbf{d}_{0}^{H}}{\sigma_{i}} \mathbf{v}_{i}
$$

Equation 3.25

Equation 3.25 shows that the weight vector $\mathbf{w}_{a}^{f r}$ is a weighted sum of the eigenvectors $\mathbf{v}_{i}$. This method can be applied to other algorithms as well, which results to:

1. For principal component:

$$
w_{a}^{P C}=\sum_{i=1}^{N} f_{i}^{P C} \frac{\mathbf{u}_{i}^{H} \mathbf{d}_{0}^{H}}{\sigma_{i}} \mathbf{v}_{i}
$$

Equation 3.26

2. For diagonal loading

$$
w_{a}^{d l}=\sum_{i=1}^{N} f_{i}^{d l} \frac{\mathbf{u}_{i}^{H} \mathbf{d}_{0}^{H}}{\sigma_{i}} \mathbf{v}_{i}
$$

3. For MWF

Equation 3.27

$$
w_{a}^{M W F}=\sum_{i=1}^{N} f_{i}^{M W F} \frac{\mathbf{u}_{i}^{H} \mathbf{d}_{0}^{H}}{\sigma_{i}} \mathbf{v}_{i}
$$

Equation 3.28

where $f_{i}^{P C}=0$ if $i \leq r^{P C}$ and $f_{i}^{P C}=1$ otherwise. $f_{i}^{d l}=\frac{\lambda_{i}}{\lambda_{i}+\beta^{2}}$ and $f_{i}^{M W F, r}=1-\prod_{j=1}^{r} \frac{\theta_{j}^{r}-\lambda_{i}}{\theta_{j}^{r}}$. These are the filter factors. The $\theta_{j}^{r}$ for the last filter factor are known as "Ritz values" which are the eigenvalues of $\hat{\mathbf{R}}_{x_{0}}$ in tridiagonal form, or $\hat{\mathbf{R}}_{d}$. Equations 3.26, 3.27 and 3.28 demonstrate that all weight vectors are calculated from weighted sum of eigenvectors and 
their respective filter factor. This is the relationship I wanted to show. This will not be discussed further in this thesis due to little relevance here. However if any curious reader is interested to know more, Rank-Deficient and Discrete ill-Posed Problems by P.C. Hansen is your best source.

\section{Chapter 4}

\section{Rank and sample compressions}

For STAP, the term "rank compression" refers to lowering the dimensions of operating subspace so that fewer adaptive degree of freedoms are required to process the data. This is highly desirable because computational complexities are proportional to filter rank thus reducing filter rank would save time and cost. The MWF has been shown that it can operate at lower rank than principal components and cross spectral metric in $\operatorname{STAP}(16,35,78,55)$. This translates to strong interest in how to implement MWF for STAP applications. 
Sample compression is another desirable trait. Sample compression reduces training sample requirements. In military applications, some system must operate in high tech environment with strong interferences. Typically in these "sample starved" environments, good training samples are difficult to acquire. Algorithms that can operate in these scenarios continue to draw strong interest from the industry. Unfortunately MWF excellent rank compression does not translate into noticeable sample compression.

In this chapter we will explore factors that contribute to rank and sample compressions. We will analyze MWF and understand why it offers superior rank compression than PC and CSM. We will also explain why this rank compression does not translate to sample compression.

\subsection{Sample Compression}

Typically $2 \mathbf{N}$ ( $\mathbf{N}$ is number of adaptive DoF) samples are needed for full rank minimum variance distortion-less response (66). But good number of quality samples is generally difficult to acquire, especially for large arrays. However research has shown that with reduce rank techniques, sample support does not follow the " $2 \mathrm{~N}$ " rule $(2,31,37,75)$. For example with diagonal loading, when there are $N_{s}$ interferers, good performance can be achieved with $2 N_{s}$ samples as opposed to $2 \mathrm{~N}$ samples $(71,75)$, where $N_{s}$ is less than $\mathbf{N}$ and corresponds to the number of large eigenvalues (i.e. 
interferers). This can be significant reduction in sample support requirement and it holds true for $\mathrm{PC}$ as well.

Unlike PC, the MWF rank compression is lower than the number of significant interferers. It was the hope of researchers that MWF excellent rank compression would translate to excellent sample compression as well, unfortunately this did not materialize. So the question became: was MWF sample requirement proportional to number of MWF stages or the number of significant interferers in the environment? In this thesis I will explain that MWF sample requirement is more related to the number of interferers.

Recall that "sample requirement" is the number of samples needed for SINR performance to be within 3dB of the optimum MVDR-SMI response and recall that MSE is

$$
M S E=\mathbf{w}^{H} \mathbf{R}_{\mathbf{x}} \mathbf{w}
$$

Equation 4.1

where $\mathbf{R}_{\mathbf{x}}$ is the true covariance matrix and $\mathbf{w}$ is the calculated weight vector. The SINR is related to the MSE by

$$
\operatorname{SINR}=\frac{N \sigma_{s}^{2}}{M S E}
$$

Equation 4.2

where $\boldsymbol{N}$ is the number of adaptive degrees-of-freedom and $\sigma_{s}^{2}$ is the element-level desired signal power. For the sake of comparison we must normalize SINR with the optimum $\operatorname{SINR}_{\text {opt }}$ or 


$$
\rho=\frac{\operatorname{SINR}}{\operatorname{SINR}_{\text {opt }}}
$$

Equation 4.3

Combining Equations 4.1 and 4.2 with 4.3 gives us

$$
\rho=\frac{M M S E}{M S E}
$$

\section{Equation 4.4}

where MMSE is the minimum mean square error, which is associated with the optimum MVDR weight vector. With these metrics defined, I will simulate with the parameters in Table 1.

Table 1: Simulation parameters 1

\begin{tabular}{|c|c|}
\hline Parameter & Value \\
\hline N elements & 8 \\
\hline M pulses/CPI & 10 \\
\hline $\begin{array}{c}\mathrm{d} \text { (inter element } \\
\text { distance) }\end{array}$ & $10 \mathrm{~dB}$ \\
\hline $\begin{array}{c}\text { Clutter power } \\
\text { (CNR) }\end{array}$ & 2 \\
\hline $\begin{array}{c}\text { \# of effective } \\
\text { Jammers }\end{array}$ & $30 \mathrm{~dB}$ \\
\hline $\begin{array}{c}\text { Jammer power } \\
\text { (JNR) }\end{array}$ & $0 \mathrm{~dB}$ \\
\hline Noise power & 1 \\
\hline $\begin{array}{c}\beta \text { (aka DPCA } \\
\text { mode) }\end{array}$ & 100 \\
\hline Monte Carlos trials & \\
\hline
\end{tabular}




\begin{tabular}{|c|c|} 
CMT type & ICM \\
\hline b & 5.7 \\
\hline wind speed & $10 \mathrm{Mph}$ \\
\hline PRF & $1 \mathrm{KHZ}$ \\
\hline fc (Carrier Freq) & $1 \mathrm{GHZ}$ \\
\hline
\end{tabular}

$\mathrm{CPI}$ is the coherent processing interval. CNR and JNR are the clutter and jammer to noise ratio respectively. $\beta$ (Beta) is as described in chapter 2. CMT stands for covariance matrix tapers. We will not discuss CMT in great details here. All we need to know for now is that they account for mismatch errors such as channel mismatch, antenna dispersion and etc. ICM (internal clutter motion) is the only CMT we account for in these simulations. It is developed by Bell Labs which model errors due to clutter reflected from foliage in the air. The parameters of $b$, wind speed, PRF and carrier freq are all needed to derive the impact of ICM. From these four parameters one worth noting is the pulse repetition frequency (PRF). PRF controls the frequency of transmitting pulses. In many practical applications, signal trains are generated in multiple PRFs (a.k.a. a dwell) to resolve range and Doppler ambiguities. Though we will not be using dwells for our simulation, it is worth noting their importance.

Figure 22 below shows the normalized SINR performance vs sample support for two randomly placed jammers. 
A.) Normalized SINR vs Samples for MWF

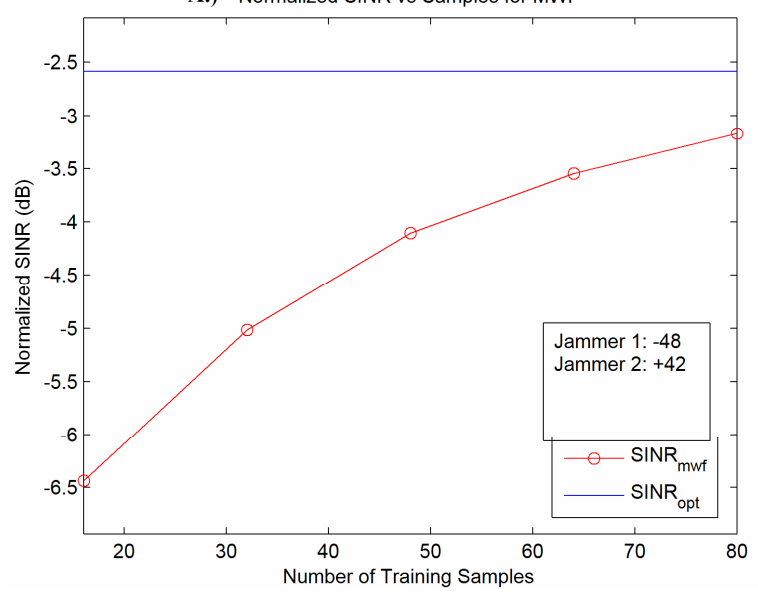

B.) Normalized SINR vs Samples for MWF

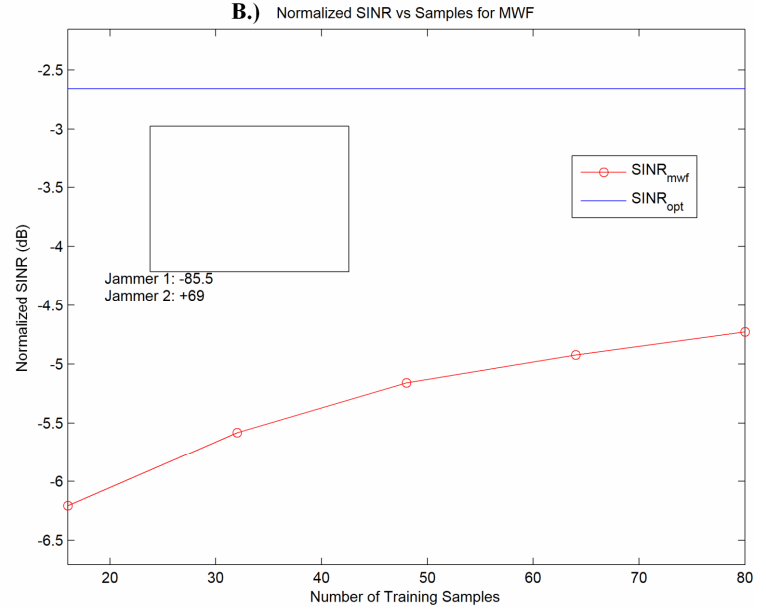

Figure 22: Normalized SINR for two jammers

Though the performance of Fig 22 A.) is superior for higher sample support, both are within the desired range $(\sim 3 \mathrm{~dB})$ of optimum with approximately 30 samples. Figure 23 shows two cases with three jammers
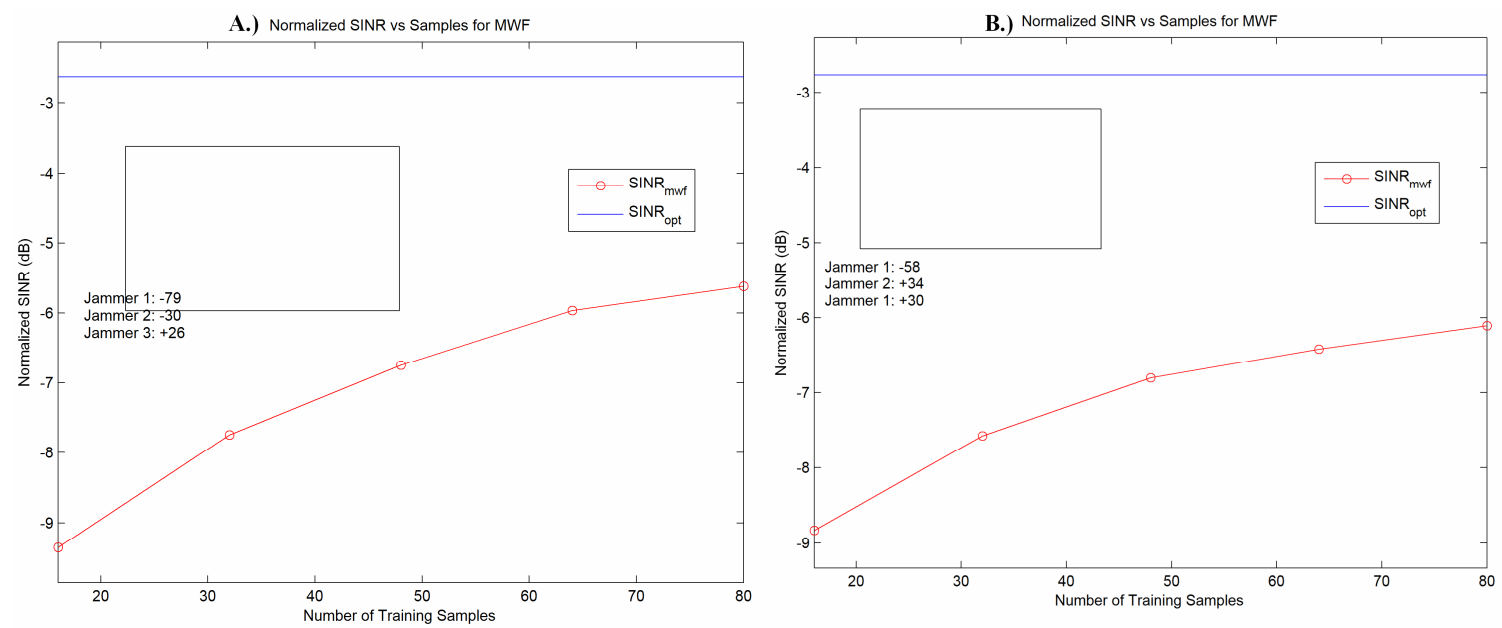

Figure 23: Normalized SINR for three jammers

In this case, the sample support needs to be larger than 80 to achieve desired SINR performance.

Figure 22 and 23 demonstrates the impact of interferers in sample support requirements. Clearly, more samples are needed if more 
interferers are present. However this also depends on other factors such as array type, closely spaced signals and etc. Though MWF sample support requirements failed to live up to our hopes in the case of three or more jammers, it is still more favorable than alternatives like PC. Principal component in its original form generally follows the " $2 \mathbf{N}$ " sample rule, while MWF frequently requires only $\mathbf{N}$ samples.

\subsection{Rank Compression}

In this section we will examine multi-stage Wiener filter (MWF) rank compression and factors that affects it. We will first detail few mechanisms that contribute to rank compression then we will describe MWF equation base to understand why its compression is superior to principal component (PC) and cross spectral metric (CSM). Finally, comparisons of PC-SD and MWF rank compression will be simulated.

Consider the unconstrained filter shown in Figure 24.

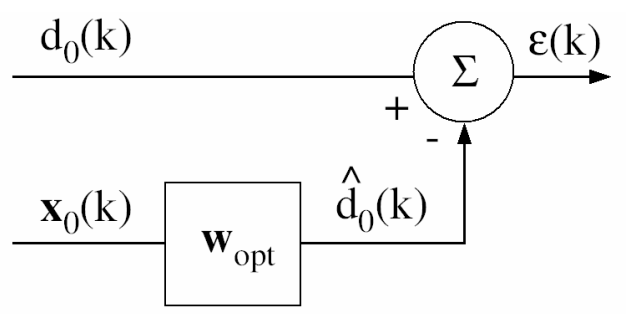

Figure 24: Classic Wiener filter 
The goal of an adaptive filter is to provide the best possible estimate of the desired signal based on the observed data. Thus we are interested in comparing the number of adaptive degrees of freedom (or rank) needed by the MWF and PC. For principal components, the rank of the PC filter is equal to the number of significant eigenvalues of the data covariance matrix that are used in the weight vector calculations. For the MWF, the rank is determined by the number of MWF stages used.

Before we explore MWF rank compression more fully we should first understand a few factors that contribute to rank compression. Some of these factors can be considered as "natural" while others are planned.

\subsubsection{Closely spaced signals}

We noted earlier that the number of interferers is related to number of significant eigenvalues which in turn is mirrored in the rank of the received signal. However there are many instances that this is not true, and the number of significant eigenvalues of the data covariance matrix can be less than the signal rank. One such case is closely spaced signals.

Consider an $\mathbf{N}$ element array and two jammers arriving from different angles. The resulting jammer covariance matrix $\mathbf{R}_{j}$ is

$$
\mathbf{R}_{j}=\sigma_{1}^{2} \mathbf{v}_{\theta_{1}} \mathbf{v}_{\theta_{1}}^{H}+\sigma_{2}^{2} \mathbf{v}_{\theta_{2}} \mathbf{v}_{\theta_{2}}^{H}
$$

Equation 4.5 
The $\mathbf{R}_{j}$ can be decomposed using the eigenvalue decomposition (EVD)

$$
\mathbf{R}_{j}=\sum_{i=1}^{2} \lambda_{i} \mathbf{v}_{i} \mathbf{v}_{i}^{H}
$$

\section{Equation 4.6}

The eigenvalues can be expressed in terms of jammer power $\sigma_{i}^{2}$ and its array manifold vector $\mathbf{v}_{\theta_{i}}$. A closed form solution was found in reference (70)

$$
\lambda_{1(2)}=\frac{1}{2} N\left(\sigma_{1}^{2}+\sigma_{2}^{2}\right)\left(1 \pm \sqrt{1-\frac{4 \sigma_{1}^{2} \sigma_{2}^{2}\left(1-\left|B_{c}^{(1,2)}\right|^{2}\right)}{\left(\sigma_{1}^{2}+\sigma_{2}^{2}\right)^{2}}}\right)
$$

Equation 4.7

is a function of signal separation where $B_{c}^{(1,2)}$ is the spatial correlation between signal 1 and 2 .

$$
B_{c}^{(1,2)}=\frac{\mathbf{v}_{\theta_{1}}^{H} \mathbf{v}_{\theta_{2}}}{N}
$$

\section{Equation 4.8}

Equation 4.7 showed that when confronting two jammer signals, if the spacing between the signals is wide then there is very little interaction between them. However, if the spacing between the signals is narrow, then the two signals merge into a dominant eigenvalue. This illustrates that closely spaced signals can cause the number of significant eigenvalues to be less than the number of signals received and thus lead to reduced rank data that require fewer adaptive degrees of freedoms to 
process. In practice, closely spaced signals or separation that is less than one half power beam-width (HPBW) result in spatial correlation between the signals. This means that if our airborne platform is positioned at the right angle then many received interferers can be condensed into just a few.

\subsubsection{Filter constraints}

Another factor that contributes to rank compression is filter constraints. We have already discussed one such constraint, the MVDR or $\mathbf{w}^{H} \mathbf{s}=1$. Constraints like this provide opportunities to reduce rank for adaptive array processing.

Consider the general side-lobe canceller (GSC) shown in Figure 25. The top path is a physical example of the distortion-less constraint $\mathbf{w}^{H} \mathbf{s}=1$. The input data $\mathbf{x}$ is filtered through two blocks $\mathbf{s}$ and $\mathbf{B}$. The block $\mathbf{s}$ corresponds to the desired steering vector hence its output corresponds to the desired main lobe response. Block B is similar except its output are the side lobe responses. Jammers are suppressed by steering side lobe nulls toward their directions and can be completely annihilated if they align precisely as shown in Chapter 2. In such case, no adaptive stages would be required. 


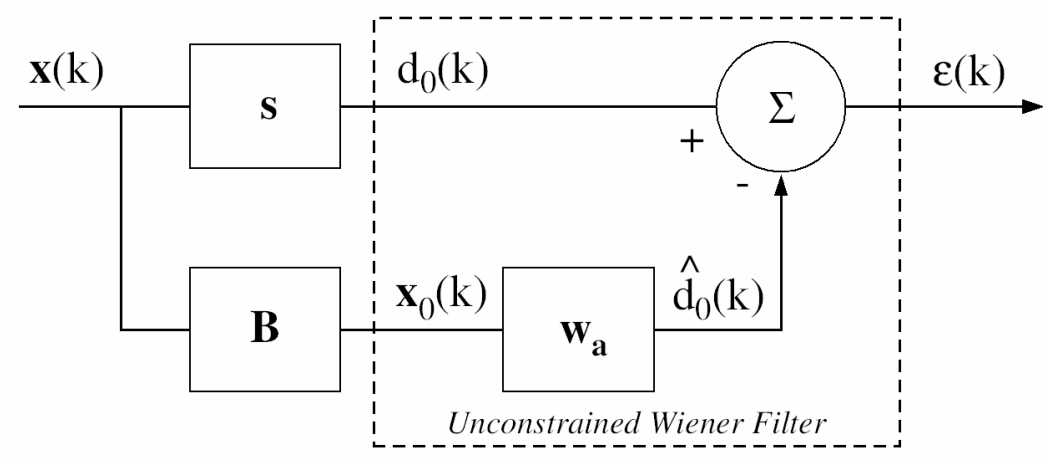

Figure 25: General side-lobe canceller

Unfortunately this is not the case in practice. Nulls cannot be steered to perfection therefore interference energy leaks to all channels. As the spatial separation between the jammers and nulls increase, more MWF and PC adaptive stages would be required to reject the interference.

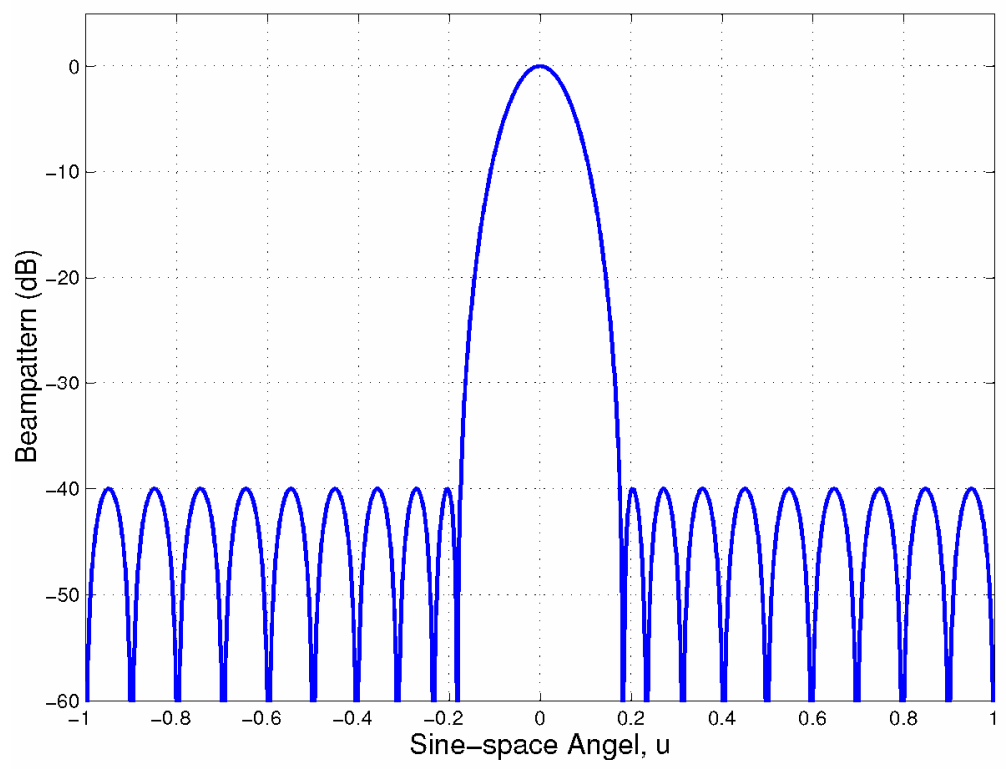

Figure 26: beam-former with Chebychev taper

\subsubsection{Pattern constraints}


The MVDR constraint is one of many that can be applied. Another example is the Chebychev side lobe constraint (36). Figure 26 is a demonstration; notice all side lobe levels are capped. As discussed earlier, modern antenna designers favor reduced side lobe levels to a wider main beam. This is due to observations that majority of interference are received by the side lobes, hence if these responses are suppressed than the received interference power are suppressed as well. In practice this translates to rank compression. MWF typically benefit more from this than PC.

\subsubsection{Rank compression in MWF}

In this section I will detail why MWF offer superior rank compression than

PC. Recall that the received data covariance matrix can be decomposed into

$$
\mathbf{R}_{\mathbf{x}_{0}}=\mathbf{V} \Lambda \mathbf{V}^{H}=\sum_{i=1}^{N} \lambda_{i} \mathbf{v}_{i} \mathbf{v}_{i}^{H}
$$

Equation 4.8

Principal components algorithm reconstruct the data covariance matrix into a lower rank version by using the eigenvector basis or

$$
\begin{gathered}
\mathbf{R}_{\mathbf{x}_{0}}=\sum_{i=1}^{r^{P C}} \lambda_{i} \mathbf{v}_{i} \mathbf{v}_{i}^{H} \\
\text { Equation } 4.9 \\
\varepsilon\left(\mathbf{R}_{\mathbf{x}_{0}}, r^{P C}\right)=\operatorname{span}\left\{\mathbf{v}_{1}, \mathbf{v}_{2}, \ldots \mathbf{v}_{r^{P C}}\right\}
\end{gathered}
$$

Equation 4.10 
where the eigenvalues/eigenvectors are sorted in descending order and $r^{P C}<N$. Therefore only the strong eigenvalues/eigenvectors are retained. But note that the desired steering vector $\mathbf{s}$ is not taken into consideration in the formation of the reduced rank interference covariance. This makes the general PC algorithm known as a data-dependent signalindependent algorithm.

MWF does not use the eigenvector basis, instead it uses the Krylov basis or

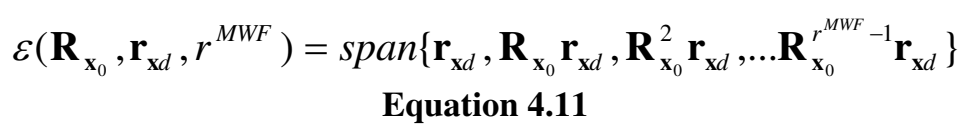

where $\mathbf{r}_{\mathbf{x} d}$ is the cross correlation vector between the data $\mathbf{x}$ and desired output $d$ and $r^{M W F}$ is the set rank. Expanding $\mathbf{r}_{\mathbf{x} d}$ we have

$$
\mathbf{r}_{\mathrm{x} d}=\alpha_{1} \mathbf{v}_{1}+\alpha_{2} \mathbf{v}_{2}+\ldots+\alpha_{N} \mathbf{v}_{N}
$$

Equation 4.12

where $\alpha_{i}$ is the cross correlation coefficient between the desired signal and the eigenvector $\mathbf{v}_{\mathrm{i}} \cdot \mathbf{r}_{\mathrm{x} d}$ is the first basis of the Krylov subspace. The second is $\mathbf{R}_{\mathbf{x}_{0}} \mathbf{r}_{\mathbf{x} d}$. If we expand $\mathbf{R}_{\mathbf{x}_{0}} \mathbf{r}_{\mathbf{x} d}$ into

$$
\mathbf{R}_{\mathbf{x}_{0}} \mathbf{r}_{\mathbf{x} d}=\mathbf{V} \Lambda \mathbf{V}^{H}\left(\alpha_{1} \mathbf{v}_{1}+\alpha_{2} \mathbf{v}_{2}+\ldots+\alpha_{N} \mathbf{v}_{N}\right)
$$

Equation 4.13

but recall that eigenvectors are orthonormal to each other except with itself, or 


$$
\mathbf{v}_{i}^{H} \mathbf{v}_{j}=\left\{\frac{1_{-} i f_{-} i=j}{0_{-} i f_{-} i \neq j}\right.
$$

Equation 4.14

thus

$$
\mathbf{V}^{H} \mathbf{r}_{\mathbf{x} d}=\left[\alpha_{1}, \alpha_{2}, \ldots \alpha_{N}\right]^{T}
$$

Equation 4.15

Equation 4.13 can be simplified to

$$
\mathbf{R}_{\mathbf{x}_{0}} \mathbf{r}_{\mathbf{x} d}=\alpha_{1} \lambda_{1} \mathbf{v}_{1}+\alpha_{2} \lambda_{2} \mathbf{v}_{2}+\ldots+\alpha_{N} \lambda_{N} \mathbf{v}_{N}
$$

Equation 4.16

Equations 4.12 and 4.16 are the first and second Krylov basis sets. The higher order Krylov basis can be defined as

$$
\mathbf{R}_{\mathbf{x}_{0}}^{k} \mathbf{r}_{\mathbf{x} d}=\alpha_{1} \lambda_{1}^{k} \mathbf{v}_{1}+\alpha_{2} \lambda_{2}^{k} \mathbf{v}_{2}+\ldots+\alpha_{N} \lambda_{N}^{k} \mathbf{v}_{N}
$$

where

$$
\mathbf{R}_{\mathbf{x}_{0}}^{k}=\mathbf{V} \Lambda^{k} \mathbf{V}^{H}
$$

Equation 4.18

Looking closely at Equation 4. 17, we find that each of the Krylov basis vectors is a weighted sum of the eigenvectors. This is similar to principal components (PC). In fact if all $\alpha_{i}=1$ the resulting rank compression is same as PC. Since these weight values are the function of both eigenvalue and the cross correlation coefficient then the MWF rank 
compression will always be better than or equal to its PC counterpart because $\alpha_{i} \leq 1$.

Therefore in Krylov subspace, if $r^{M W F}=N$ then all $\mathrm{N}$ Krylov basis vectors are kept and the full $\mathrm{N}$ dimensional space is spanned. But if $r^{M W F}<N$ then the Krylov subspace dimension can be reduced based on low eigenvalue, low correlation, or a combination of both.

In practice, it is observed that environment with low power interferers are well handled by MWF rank compression due to the low $\alpha_{i} \lambda_{i}^{k}$ product. Environments with closely spaced interference sources are also good candidate for MWF because their close proximity creates a bifurcation into a dominant eigenvector and a weak one. These weaker eigenvectors becomes additional candidates for rank compression by the MWF.

\subsubsection{Practical factors to consider}

Before we get to our STAP simulations there are more observed factors that impact rank compression. First it is observed that increased sample support does not yield increased rank compression. In some cases, rank increased as sample support increased. The reason for this is that more samples gives us more accurate estimate of basis vectors that prior was considered marginal contributors but are now more significant. Another 
factor is that eigenvalues of noise level signals are typically poorly estimated. This inability to accurately define noise subspace is one of the motivations to use reduce rank transformations (RRT). In that note, environments with low power interference typically provide best MWF rank compression. PC-SD algorithm is also a good choice in these environments because it too considers jammer power levels, however much less pronounced than MWF. In practical applications, detrimental effects of covariance matrix tapers (CMT) should not be ignored. They typically do not affect large eigenvalues but can create small ones. This is another reason why MWF is desired because of it's excellence in suppressing these new CMT created eigenvalues.

One of the worst case scenarios in STAP is the sudden appearance of jammers in the environment. This can significantly increase rank because jammers contaminate all doppler bins and infects the sampled data. The typical solution is to estimate the directions of these new jammers and quickly adjust the pattern to nullify them before they cause too much damage. However this solution can be inefficient, as enemies could turn jammers on and off at will, hence why algorithms that can effectively suppress the effects of new jammers draw strong interest. In contrary to sudden appearance of new jammers, the sudden disappearance of jammers does not detriment performance and is of little concern. 


\subsection{STAP simulation}

We can now examine MWF and PC rank compression for space-time adaptive processing (STAP). As mentioned earlier, STAP environment includes three types of undesirable interference signals: jammers, noise, and clutter. Figure 27 shows the eigenspectra of two environments. One environment includes 2 randomly placed jammers of $30 \mathrm{~dB}$ jammer to noise ratio (JNR), 10dB clutter to noise ratio (CNR), noise at OdB and ICM effects. The second environment is the same as the first minus the jammers. 


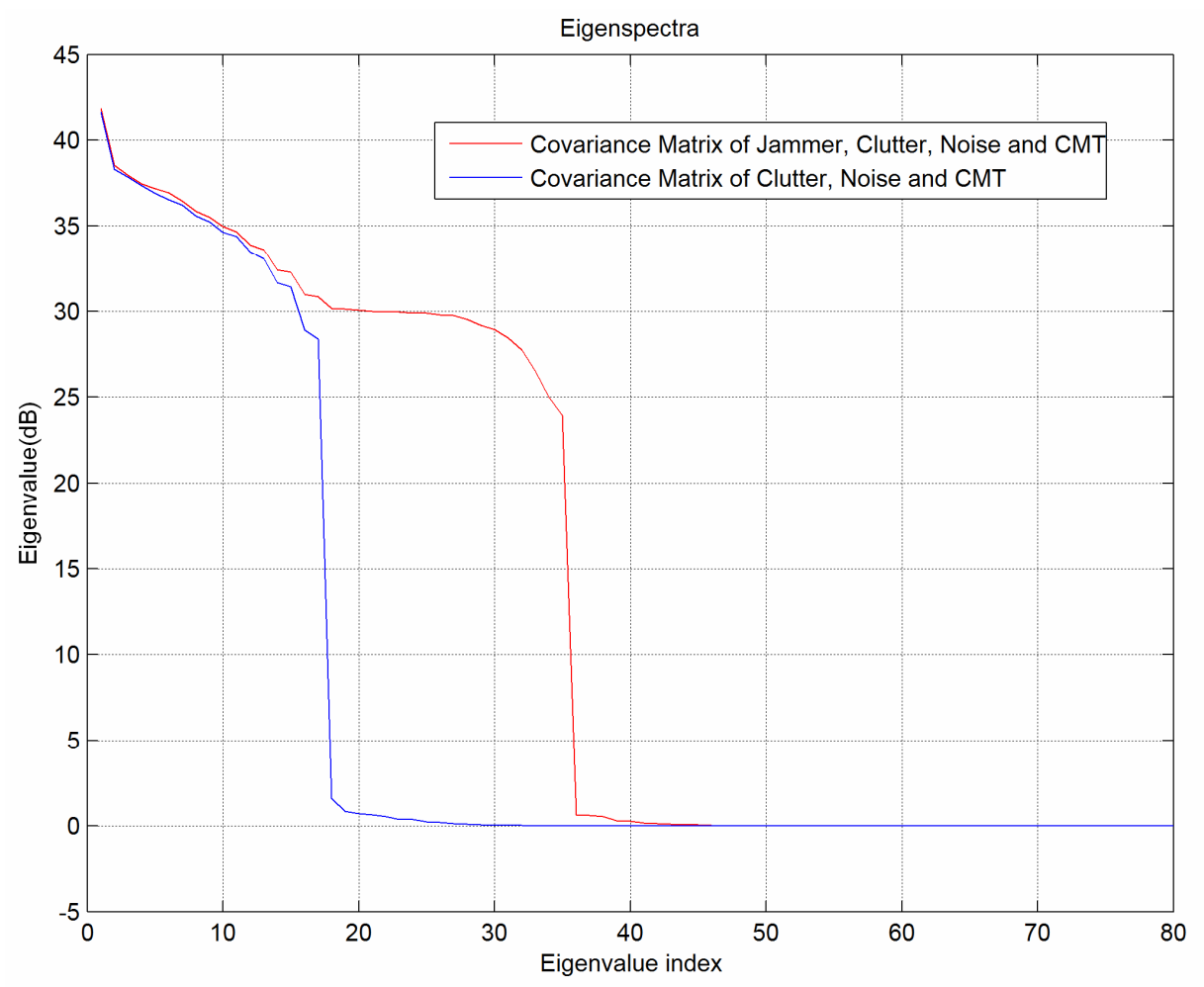

Figure 27: Eigenspectra

This eigenspectra reveals the number of significant eigenvalues in the interference covariance matrix. The matrix without the jammers (blue curve) is dominated by clutter and this gives us perspective on the role clutter play. The Brennan's rule or

$$
r_{c}=\mathbf{N}+(\mathbf{M}-1) \beta
$$

\section{Equation 4.19}

is an generally accepted guideline when dealing with clutter. It estimates the number of significant eigenvalues created by clutter with only three parameters: the number of elements $\mathbf{N}$, the number of pulses $\mathbf{M}$, and $\beta$ which was discussed earlier. With our simulating parameters in Table 2, 
the calculated clutter rank is 17. This is near the simulated result of 18. The added rank could be the result of covariance matrix tapers (CMT).

The second curve (red) demonstrates the impact of two 30dB JNR jammers on the eigenspectra. As stated earlier jammer signals contaminates all channels. From simulation we see that contamination resulted in many more strong eigenvalues (i.e. rank). Comparing the two curves of Figure 27 we see that adding two jammers have doubled the rank and hence the number of needed adaptive degree of freedoms to cancel out the interference. Therefore it is highly desirable to implement reduce rank transformations (RRT) to lower processing cost.

We can now evaluate the performance of MWF and PC-SD. Our simulation parameters are defined in Table 2.

Table 2: Simulation parameters 2

\begin{tabular}{|c|c|}
\hline Parameter & Value \\
\hline N elements & 8 \\
\hline $\begin{array}{c}\text { M pulses/CPI } \\
\text { distance) }\end{array}$ & $1 / 2$ \\
\hline $\begin{array}{c}\text { Clutter power } \\
\text { (CNR) }\end{array}$ & $10 \mathrm{~dB}$ \\
\hline $\begin{array}{c}\text { \# of effective } \\
\text { Jammers }\end{array}$ & 2 \\
\hline $\begin{array}{c}\text { Jammer power } \\
\text { (JNR) }\end{array}$ & $50 \mathrm{~dB}$ \\
\hline Noise power & $0 \mathrm{~dB}$ \\
\hline $\begin{array}{c}\beta \text { (aka DPCA } \\
\text { mode) }\end{array}$ & 100 \\
\hline \begin{tabular}{c} 
Monte Carlos trials \\
\hline
\end{tabular}
\end{tabular}




\begin{tabular}{|c|c|} 
CMT type & ICM \\
\hline b & 5.7 \\
\hline wind speed & $10 \mathrm{Mph}$ \\
\hline PRF & $1 \mathrm{KHZ}$ \\
\hline fc (Carrier Freq) & $1 \mathrm{GHZ}$ \\
\hline
\end{tabular}

Figure 28 shows MWF and PC-SD performance against two jammers located at angles of (-72 23) degrees.

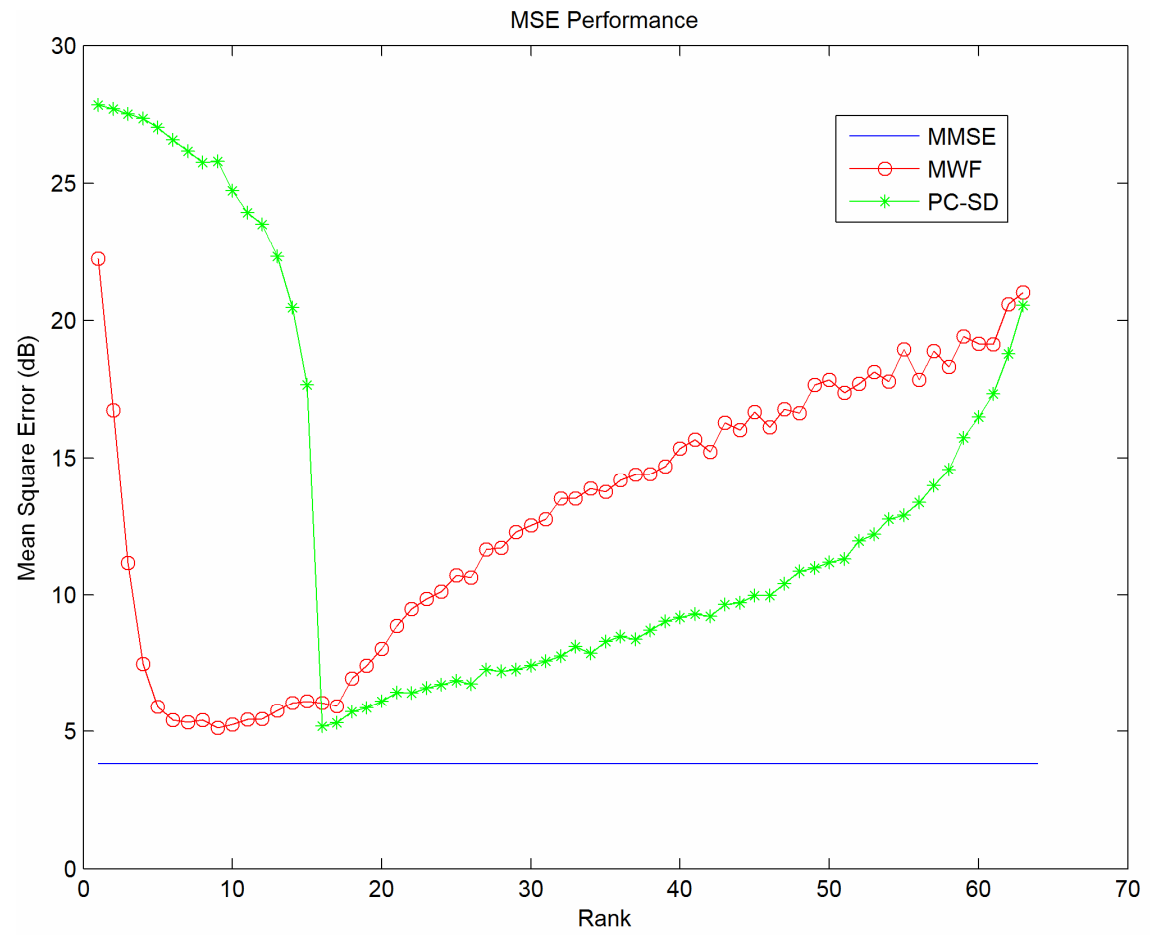

Figure 28: MWF vs PC-SD w/ 50dB JNR and 10dB CNR

PC-SD performance reaches lowest MSE at rank of 16 , this means it needs 16 adaptive degrees of freedoms (ADoF) to suppress the interference to achieve MVDR. In contrast, MWF only needs 9 ADoF to accomplish the same. Notice that MWF also offers more flexibility in rank selection. As graph shows, MWF's MSE performance of ranks from 5 to 17 are all well 
within $3 \mathrm{~dB}$ range of minimum mean square error (MMSE). This means that the MWF process can stop anywhere within stages 5 to 17 and still yield acceptable result. This type of flexibility is highly desirable.

Now let us vary the JNR. While holding CNR at 10dB we decrease the JNR from $50 \mathrm{~dB}$ (Fig 29 A.) to $20 \mathrm{~dB}$ (Fig 29 D.). Figure 29 shows the MSE performances. Rank selection for PC-SD seems unaffected by the JNR changes, however MWF shows dramatic changes.
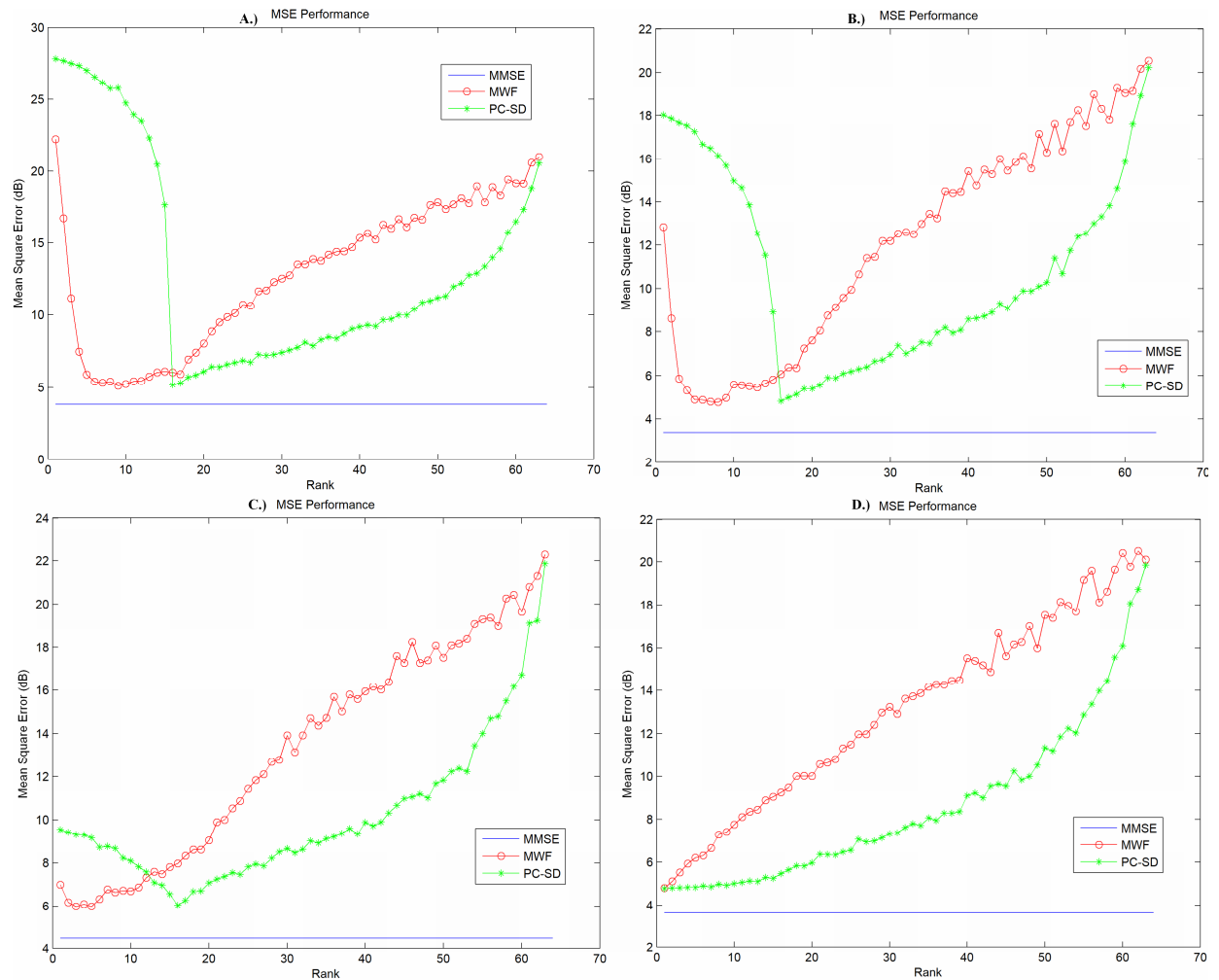

Figure 29: MWF vs PC-SD for various JNR and 10dB CNR

Table 3 shows the rank selections of MWF and PC-SD save part D. MWF adapts to the interference levels and adjust to its rank selection to 
received jammer power while PC-SD makes no adjustments. MWF adaptability in this case is desirable given that in practical situations the environment is constantly changing. In addition, MWF rank selections are less than its PC-SD counterparts which means that it could be done faster.

Table 3: Rank selections for varying JNR

\begin{tabular}{|c|c|c|c|c|}
\hline Case & $\begin{array}{l}\text { JNR } \\
\text { (dB) }\end{array}$ & $\begin{array}{l}\text { CNR } \\
\text { (dB) }\end{array}$ & $\begin{array}{c}\text { MWF } \\
\text { Rank } \\
\text { Selection }\end{array}$ & $\begin{array}{c}\text { PC-SD } \\
\text { Rank } \\
\text { Selection }\end{array}$ \\
\hline A & 50 & 10 & $5-17$ & $16-30$ \\
\hline B & 40 & 10 & $3-16$ & $16-30$ \\
\hline C & 30 & 10 & $1-12$ & $16-24$ \\
\hline
\end{tabular}

Figure 30 and Table 4 shows rank selection for environments where CNR varies from $40 \mathrm{~dB}$ (Fig $30 \mathrm{~A}$.) to $10 \mathrm{~dB}$ (Fig 30 D.) while JNR is constant at 50dB. In this case neither rank selection changes much, however MWF still offers lower rank selection. 

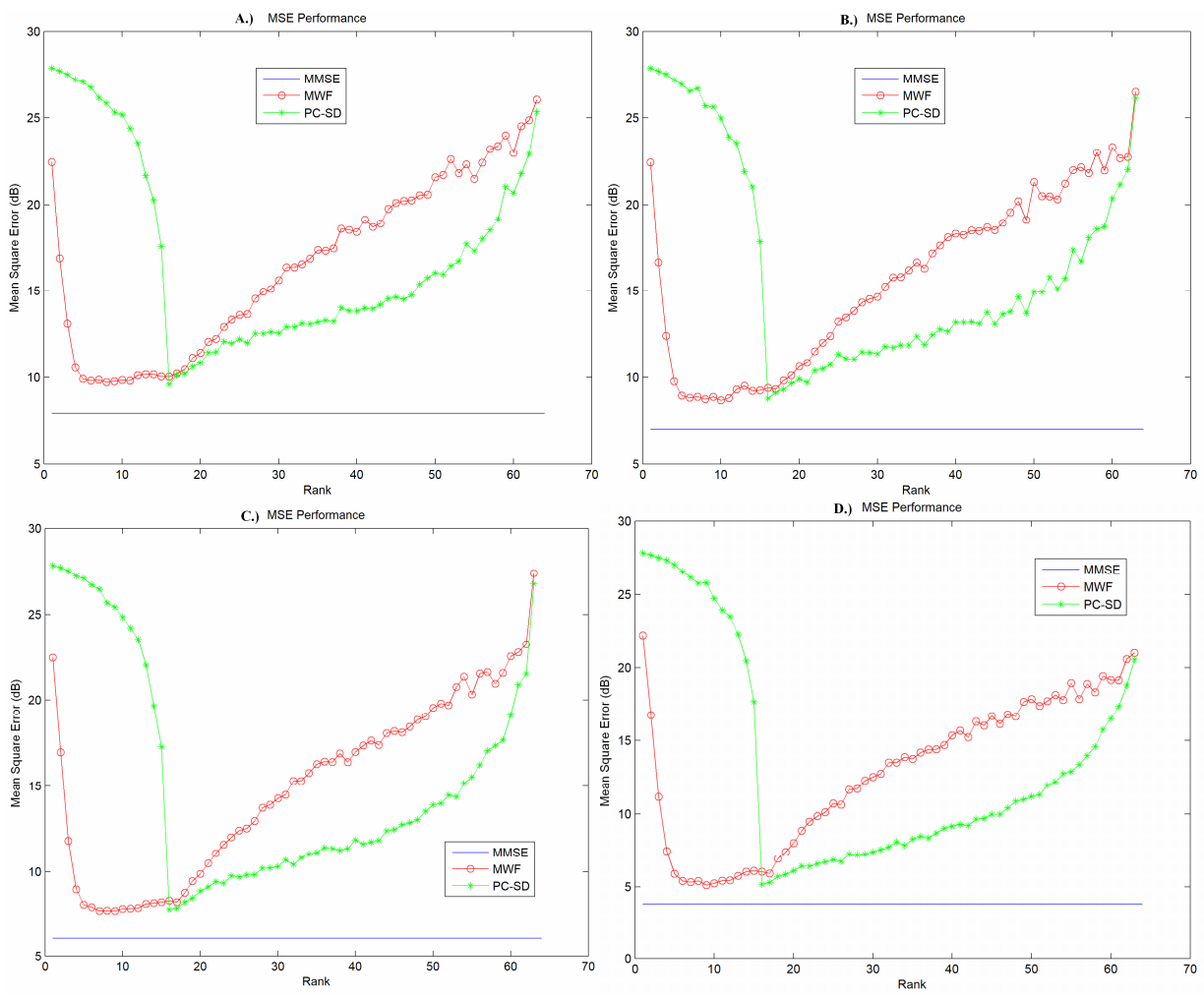

Figure 30: MWF vs PC-SD w/ 50dB JNR and various CNR

Table 4: Rank selection for varying CNR

\begin{tabular}{|c|c|c|c|c|}
\hline Case & JNR & CNR & MWF & PC-SD \\
& $(\mathbf{d B})$ & $(\mathbf{d B})$ & $\begin{array}{c}\text { Rank } \\
\text { Rank }\end{array}$ & $\begin{array}{c}\text { Selection } \\
\text { Selion }\end{array}$ \\
\hline A & 50 & 20 & $5-17$ & $16-21$ \\
\hline B & 50 & 30 & $4-20$ & $16-21$ \\
\hline C & 50 & 40 & $5-19$ & $16-21$ \\
\hline
\end{tabular}

\subsection{Chapter Summary}

In this chapter we explored the concepts of rank and sample compressions. Simulation showed that MWF offered superior rank 
compression than PC-SD, especially in environments where jammer powers are lowered to $30 \mathrm{~dB}$. MWF demonstrated that it can adapt its rank selections to the environment but PC-SD did not. MWF did not significantly reduce sample requirements as hoped. In all instances, NXM samples were required to have an adequate estimate of the interference covariance matrix.

There are two things we should clarify before we move onto the next chapter. First, generating the MSE performance graphs shown in this chapter are not possible in practice. They are acquired in our simulation because we know exactly what the interference covariance matrix is in our simulated environment, but in practice that would require infinite number of samples which is practically impossible. As a result, our optimum rank selection would be more or less "blind". Second, the majority of ranks did not achieve our desired minimum variance distortionless response (MVDR). In both PC-SD and MWF, MSE performance degraded further as ranks increased beyond the optimum rank. For case in Figure 28, only 12 out of 64 possible ranks yielded acceptable results. If we blindly select our process rank, the probability of failure would be $81 \%$. Fortunately there are ways to improve our odds. These methods are known as "soft stop" and "hard stop" techniques and they will be explained in the next two chapters. 


\section{Chapter 5}

\section{Soft stops for MWF}

The term "soft stop" is derived from the similar intangible developments yielded the term software. By definition, both terms describes something "that cannot be touched". The meaning software now broadens to comprise more than just computer programs; in contrast, soft stop is specifically tailored to techniques used to enhance space-time adaptive processing (STAP).

As the name implies, soft stop achieves its goals through manipulation of software, or in our case, data. Recall from earlier that received sample data $\mathbf{x}$ is collected by our array so we can form a sampled interference covariance matrix $\hat{\mathbf{R}}_{\mathbf{x}}=\mathbf{E}\left[\mathbf{x} \mathbf{x}^{H}\right]$ that will have the same statistical properties of the original sample. This sampled covariance matrix can be altered and manipulated to achieve MVDR as shown earlier with PC-SD and MWF. But rather than reconstructing the sampled covariance matrix with its eigenvector basis, a soft stop technique directly alters the content within the covariance matrix itself. 


\subsection{Diagonal Loading as soft stop}

One soft stop technique is known as diagonal loading (DL).

Equation wise it is relatively simple.

$$
\tilde{\mathbf{R}}_{\mathbf{x}}=\hat{\mathbf{R}}_{\mathbf{x}}+\sigma_{L}^{2} \mathbf{I}
$$

Equation 5.1

where $\sigma_{L}^{2}$ is the user-defined loading level and $\mathbf{I}$ is the identity matrix of

same size as $\hat{\mathbf{R}}_{\mathbf{x}}$. At first glance one might ask the question of how does this loading enhance the performance of MWF and PC-SD? And does this loading contaminate our data? I will attempt to answer both.

Recall that the eigenvector decomposition (EVD) of the covariance matrix is

$$
\begin{gathered}
\hat{\mathbf{R}}_{\mathbf{x}}=\mathbf{V} \Lambda \mathbf{V}^{H} \\
\text { Equation } 5.2
\end{gathered}
$$

where $\mathbf{V}$ is the unitary matrix of orthonormal eigenvectors and $\Lambda$ is the diagonal matrix of eigenvalues. Adding a diagonal loading component results to the modified covariance matrix $\tilde{\mathbf{R}}_{\mathbf{x}}$

$$
\tilde{\mathbf{R}}_{\mathbf{x}}=\hat{\mathbf{R}}_{\mathbf{x}}+\sigma_{L}^{2} \mathbf{I}=\mathbf{V} \tilde{\Lambda} \mathbf{V}^{H}=\mathbf{V}\left(\Lambda+\sigma_{L}^{2} \mathbf{I}\right) \mathbf{V}^{H}
$$

Equation 5.3 
This equation shows that diagonal loading increases all eigenvalues equally hence it does not change its statistical properties.

\subsection{Diagonal loading examples}

To determine the best loading level, we can use a new metric known as the loading to noise ratio (LNR) or

$$
\begin{aligned}
& \mathbf{L N R}=\frac{\sigma_{L}^{2}}{\sigma_{n}^{2}} \\
& \text { Equation } \mathbf{5 . 4}
\end{aligned}
$$

where $\sigma_{L}^{2}$ is the loading level at each element and $\sigma_{n}^{2}$ is its thermal noise power.

Figure 31 shows PC-SD MSE performance in an environment with 10dB CNR, four 50dB jammers located at $(-43,-72,70,30)$ degrees. 


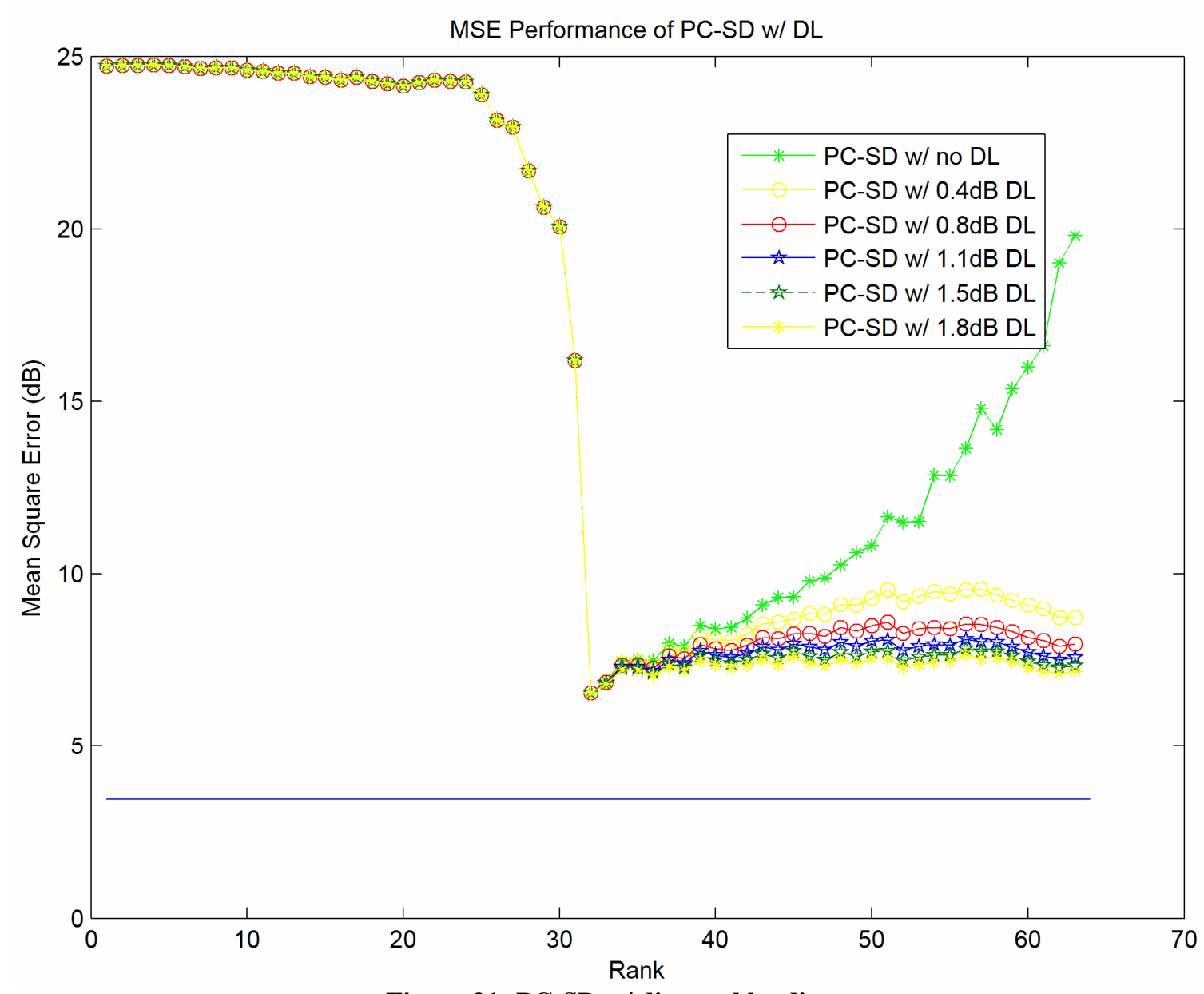

Figure 31: PC-SD w/ diagonal loading

Notice that as the loading level increased, MSE performance improved as well. In fact, for DL of $1.8 \mathrm{~dB}$, nearly all ranks above the optimum rank (31) yielded acceptable MVDR.

Figure 32 shows a similar behavior for MWF. However its acceptable rank selection is from 12 to 64 , which means that if we randomly choose a operating rank, our chance of success would be $81 \%$. 


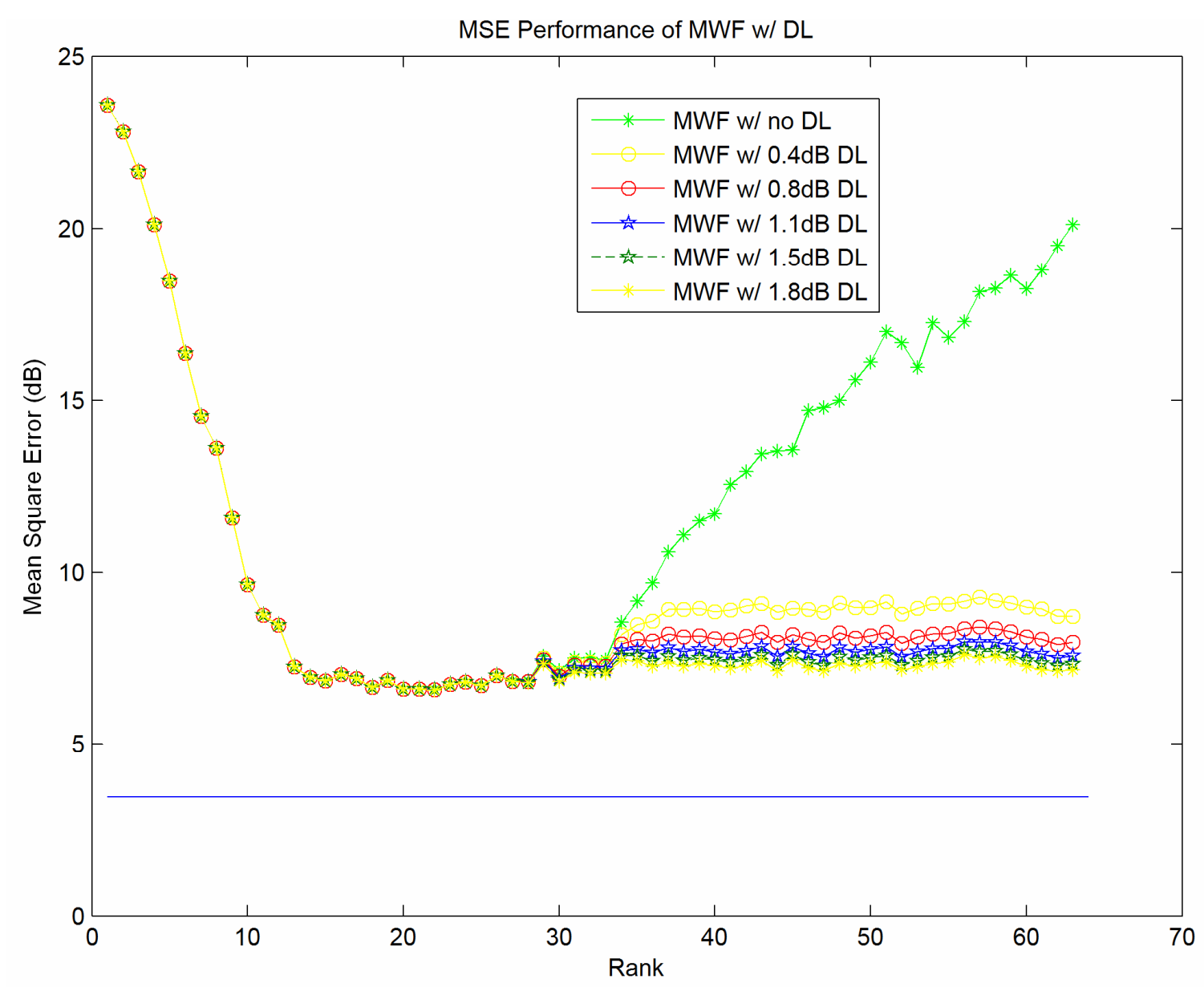

Figure 32: MWF w/ diagonal loading

Diagonal loading (DL) improves MSE performance because it increases contributions of the low eigenvalues for weight vector calculations, and these contributions improves the MSE performance past the optimum rank because those low eigenvalues are typically poorly estimated due to noise, ICM and etc.

\subsubsection{Finding the right loading level}


Though Figure 31 and 32 seems to suggest that if we continuously increase diagonal loading levels it will yield better MSE performance, this is wrong. If loading level is too high (i.e. over-load), it would make all eigenvalues significant and the MSE performance would be equivalent to a nonadaptive response (i.e. all adaptive degree of freedom are "turned off"). In contrast, if loading is too low (under-load), then MSE performance is near equivalent to a no loading scenario. A common rule of thumb in the signal processing community is to select a loading level that is approximately 5-10 dB above the noise floor.

\subsection{Chapter summary}

In summary, a properly chosen diagonal loading level will cause MWF to saturate at its peak value over a broad range of ranks, thus giving us more flexibility in rank selection. In addition, diagonal loading provides robustness against a variety of types of mismatch errors including direction-of-arrival mismatch, element perturbations, and "statistical" mismatch errors due to finite sample support $(4,70)$.

Though diagonal loading is a popular "soft stop" loading technique, it is not the only one. Other methods such as color loading and error loading (58) are also viable. 
In this chapter, we've shown a "soft stop" technique that can augment the MWF for better MSE performance. Though this improvement is impressive, it can be further improved by adding a "hard stop" technique. 


\section{Chapter 6}

\section{Hard stops for MWF}

Diagonal loading (DL) may have drawn strong connections with the first part of the name "soft stop" but one might ask how it connects with the latter part "stop". The answer is not much at all. Though DL does "stop" the degradation of MSE performance past the optimum rank, it still is hardly the "stop" we think of. A true stop is a method that can stop the MWF process at the optimum rank, or the rank that produce optimum MSE performance. Its advantage would be drastically reduced processing time and optimum SINR. As simple and beneficial DL is, it does not offer reduced processing time. For example, with DL we must still run the MWF algorithm to its full rank to guarantee satisfactory result. This means heavy processing cost.

In contrast, the term "hard stop" is true to its name. Hard stop techniques draw strong connection to both "hard" and "stop". The first part is obvious. The latter part is derived from its ability to literally stop the MWF algorithm on its track. The problem is to know when to stop. In this chapter, we will explore three hard stop techniques and reference four others. 


\subsection{White Noise Gain Constraint}

WNGC is a well known approach for selecting the amount of loading in diagonally loaded MVDR-SMI beamformers using a quadratic constraint also known as a "white noise gain constraint" (70). This technique is based on observations that many sources of error in physical systems are approximately uncorrelated from element to element and degrade performance in a manner that is similar to adding white noise to each element (4). In this perspective, array gain (AG) against white noise gives a measure of robustness. Fortunately for us, this technique can also be applied to rank selection for the MWF as well.

First, the "white noise gain" is

$$
A G^{\text {white }}=\frac{\text { SINR }^{\text {output }}}{\operatorname{SINR}^{\text {input }}}
$$

Equation 6.1

where SINR $^{\text {output }}$ is the signal to interference plus noise ratio at the output of the array,

$$
\operatorname{SINR}^{\text {output }}=\frac{N \sigma_{s}^{2}}{\mathbf{w}^{H} \mathbf{R}_{i, n} \mathbf{w}}
$$

Equation 6.2 
and SINR ${ }^{\text {input }}$ is the element level signal to interference plus noise ratio

$$
\operatorname{SINR}^{\text {input }}=\frac{\sigma_{s}^{2}}{\sigma_{i, n}^{2}}
$$

Equation 6.3

Therefore the array gain can be modified to

$$
A G^{\text {white }}=\frac{N \sigma_{i, n}^{2}}{\mathbf{w}^{H} \mathbf{R}_{i, n} \mathbf{w}}
$$

Equation 6.4

But since we are only concerned with white noise, the interference covariance matrix is

$$
\mathbf{R}_{i, n}=\mathbf{I} \sigma_{i, n}^{2}
$$

Equation 6.5

which further adjust our array gain to

$$
A G^{\text {white }}=\frac{N}{\|\mathbf{w}\|^{2}}
$$

Equation 6.6

From Equation 6.6, a uniformly weighted (i.e. $\mathbf{w}=\left(\begin{array}{lll}1 & 1 & \ldots\end{array}\right)$ ) beamformer will have the maximum value of array gain. However, if adaptive stages are added, this gain will decrease. In practice, this decrease is at first gradual as the MWF adapts against interference, but once the MWF rank exceeds the signal subspace then the adaptation will be affected by the noise subspace and the array gain will decrease appreciably. This 
deterioration will also affect other figure of merits such as the mean square error. We can limit this deterioration by limiting the decrease in $A G^{\text {white }}$. That is, we can constrain our white noise gain for the MWF in terms of the white noise gain for a conventional filter, such as

$$
10 \log \left(A G_{M W F}^{\text {white }}\right) \geq 10 \log \left(A G_{c}^{\text {white }}\right)-\eta_{\text {wngc }}
$$

Equation 6.7

The $\eta_{\text {wngc }}$ is a user defined threshold. If we substitute Equation 6.6 into Equation 6.7 we get

$$
10 \log N-10 \log \left(\mathbf{w}^{H} \mathbf{w}\right) \geq 10 \log N-10 \log \left(\mathbf{w}_{c}^{H} \mathbf{w}_{c}\right)-\eta_{\text {wngc }}
$$

Equation 6.8

where $\mathbf{w}_{c}$ is the conventional weight vector. This equation can be rewritten to

$$
10 \log \left(\mathbf{w}^{H} \mathbf{w}\right) \geq 10 \log \left(\mathbf{w}_{c}^{H} \mathbf{w}_{c}\right)-\eta_{\text {wngc }}
$$

Equation 6.9

For MWF, a normalized steering vector is used as rank one conventional weight vector or $\mathbf{w}_{c}^{H} \mathbf{w}_{c}=\mathbf{s}^{H} \mathbf{s}=1$, therefore

$$
10 \log \left(\mathbf{w}^{H} \mathbf{w}\right) \leq \eta_{\text {wngc }}
$$

Equation 6.10

Equation 6.10 is the desired form for white noise gain constraint. By its definition, if we can limit the norm square of the calculated weight vector $\|\mathbf{w}\|^{2}$ by a user defined threshold, then we can stop the performance 
degradation. This stop is translated into MWF rank selection. Please recall that different MWF with different number of stages have different weight vectors or

$$
\mathbf{w}^{m w f}=\mathbf{s}-w_{1} \mathbf{B}_{0}^{H} \mathbf{h}_{1}+w_{1} w_{2} \mathbf{B}_{0}^{H} \mathbf{B}_{1}^{H} \mathbf{h}_{2}-w_{1} w_{2} w_{3} \mathbf{B}_{0}^{H} \mathbf{B}_{1}^{H} \mathbf{B}_{2}^{H} \mathbf{h}_{3}+\ldots
$$

Equation 6.11

therefore if number of stages changes then a new weight vector must be calculated. With the white noise gain constraint, we can stop the MWF process once the norm square of that weight vector supersedes the user threshold.

Figure 33 and 34 shows two examples without DL. Figure 33 shows the first case, there are three $50 \mathrm{~dB}$ jammers located at angles of $(-76,18$, 40) degrees. 


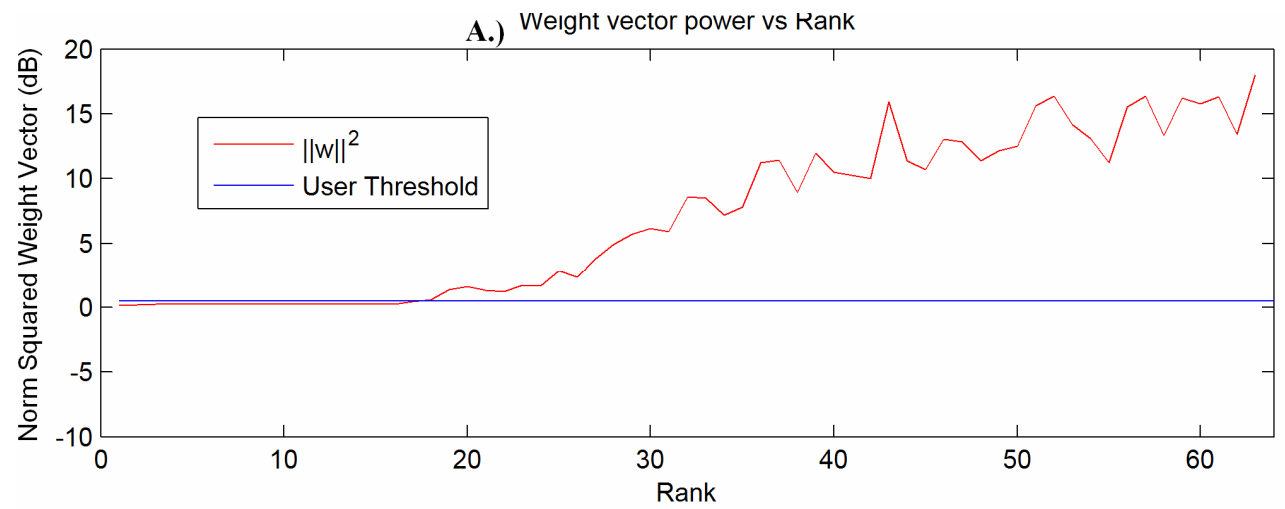

B.) MSE Performance vs Rank

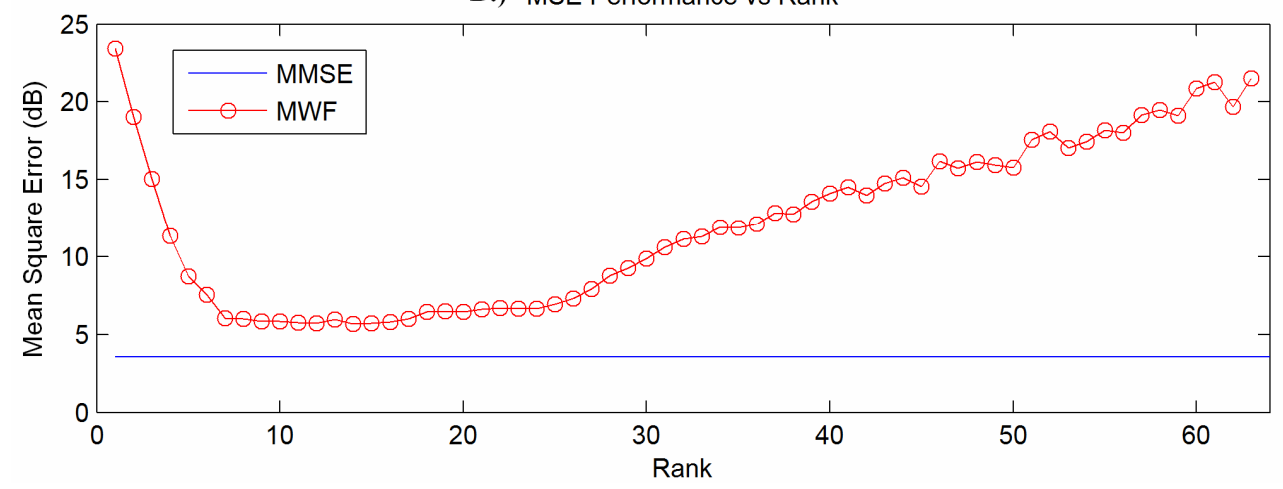

Figure 33: WNGC w/ three jammers

From part A.) we see that the calculated norm squared weight vector surpasses the $0.5 \mathrm{~dB}$ user defined threshold at rank 18 . Conversely in part B.) rank 18 offers acceptable (albeit not optimum) performance for the MWF.

In Figure 34 we will try a more difficult scenario with four jammers of similar power located at angles (-71-53 87 54) degrees. Figure 34 show that norm squared weight vector oscillates about the user threshold of $0.5 \mathrm{~dB}$. If we choose the first intersection, it would have resulted to the stopping rank of 14 , a rank that fails to meet the desired result. Clearly this example shows that an arbitrary WNGC threshold does not perform well in 
all situations, however this performance can improve if we possess prior knowledge of the environment.
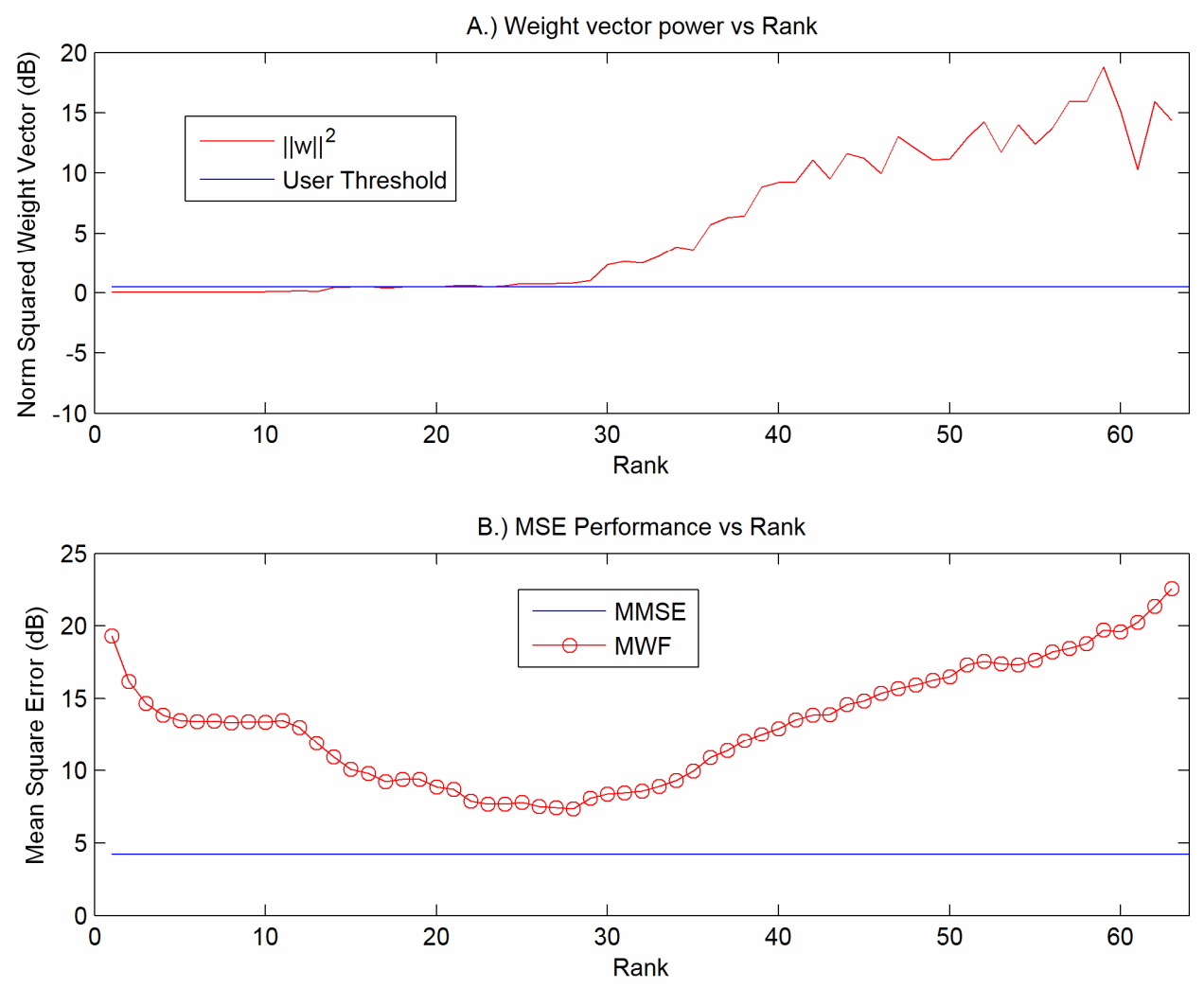

Figure 34: WNGC w/ four jammers

\subsubsection{The preferred ranks}




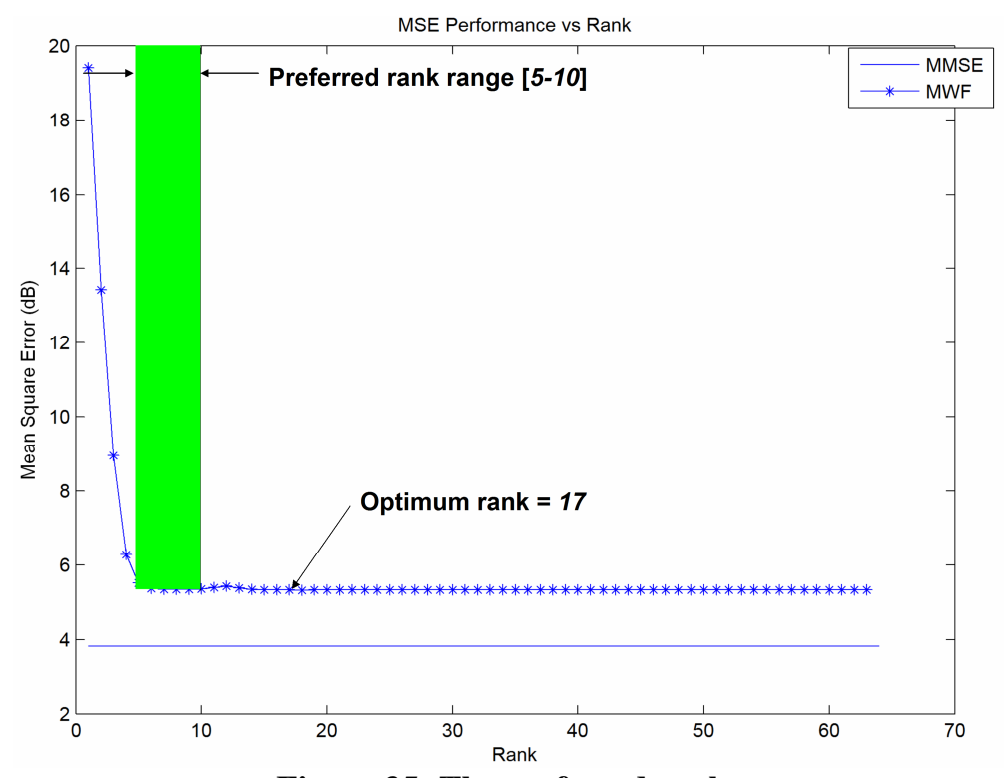

Figure 35: The preferred ranks

Figure 35 shows an example of 10dB diagonally loaded MWF MSE

performance. Its optimum rank is 17. From the perspective of maximizing SINR, it would be desirable to stop the MWF process at rank 17. However this adds unnecessary processing cost that generates little benefits. The MSE difference between ranks 5 and 17 is less than $0.1 \mathrm{~dB}$. If we weigh in cost and speed, it's much more economical to select rank 5 instead of rank 17. Figure 35 demonstrates the "preferred" rank range. This range reflects the more economical option in rank selection and will be used as a metric in rank selection performance for the remainder of this thesis.

\subsubsection{Histogram of rank selection}

Table 5: 10 examples of Optimum thresholds for WNGC

\begin{tabular}{|l|l|c||c||c||c|}
\hline Jmr 1 Angle & Jmr 2 Angle & $\begin{array}{c}\text { Avg Jmr } \\
\text { Angle }\end{array}$ & $\begin{array}{c}\text { Minimum } \\
\text { Preferred } \\
\text { Rank }\end{array}$ & $\begin{array}{c}\text { Maximum } \\
\text { Preferred } \\
\text { Rank }\end{array}$ & $\begin{array}{c}\text { Preferred } \\
\text { WNGC } \\
\text { threshold }\end{array}$ \\
\hline
\end{tabular}




\begin{tabular}{|c|c|c||c||c|c|}
\hline & & & & & \\
\hline-19.079 & 12.294 & 15.686 & 5 & 7 & 0.358 \\
\hline-24.304 & 11.232 & 17.768 & 6 & 8 & 0.565 \\
\hline-27.582 & 16.490 & 22.036 & 4 & 6 & 0.093 \\
\hline-16.625 & 32.867 & 24.746 & 4 & 6 & 0.092 \\
\hline \hline-37.541 & 12.857 & 25.199 & 4 & 8 & 0.188 \\
\hline \hline-13.220 & 37.231 & 25.226 & 4 & 8 & 0.153 \\
\hline-32.205 & 21.989 & 27.097 & 5 & 7 & 0.234 \\
\hline \hline-24.857 & 29.482 & 27.170 & 4 & 7 & 0.136 \\
\hline \hline-24.579 & 30.858 & 27.719 & 4 & 7 & 0.142 \\
\hline-18.726 & 36.733 & 27.730 & 4 & 6 & 0.251 \\
\hline \hline $\begin{array}{c}\text { Average of } \\
\text { Preferred } \\
\text { Thresholds }\end{array}$ & & & & & \\
\hline \hline $\begin{array}{c}\text { Variance of } \\
\text { Preferred } \\
\text { Thresholds }\end{array}$ & & & & & $\mathbf{0 . 1 2 7}$ \\
\hline
\end{tabular}

Table 5 shows 10 examples from a 100 example study done on the preferred WNGC thresholds generated by MATLAB codes in Appendix E. In this study, two $50 \mathrm{~dB}$ jammers are randomly positioned between \pm 10 to \pm 90 degrees. This time we add a $10 \mathrm{~dB}$ of $\mathrm{DL}$. All other simulation parameters are same as in Table 2 except we run for 30 monte carlo trials. The right-most column in this table shows the WNGC thresholds that correspond to the preferred ranks. The average of these thresholds is calculated from 100 examples. 

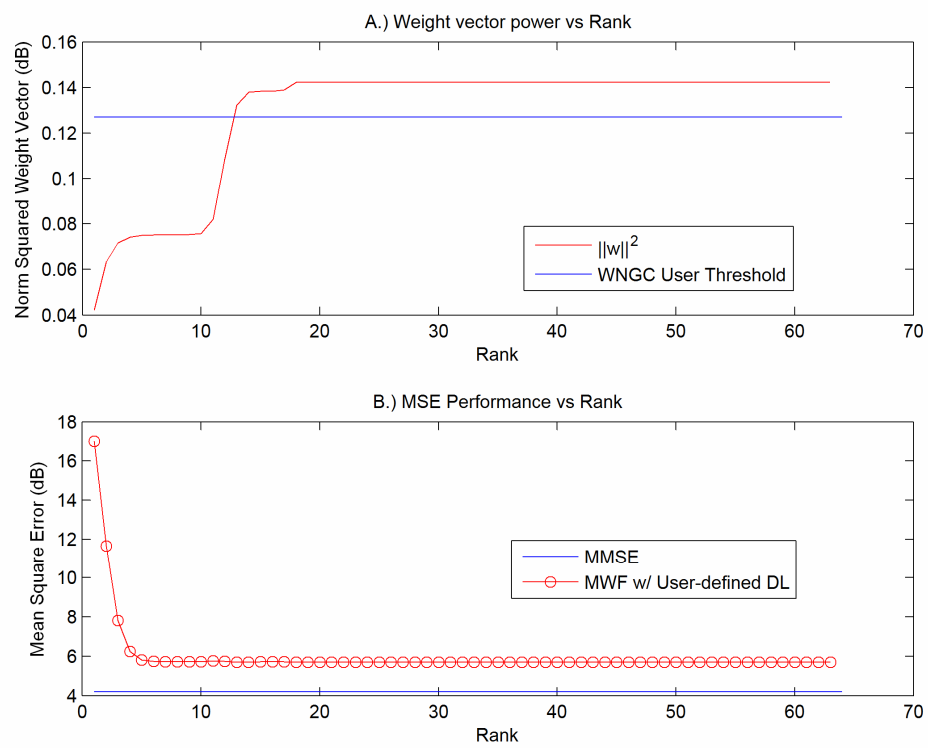

Figure 36: Example of WNGC rank selection

Figure 36 shows one example from the 100 example study. With threshold set at 0.127 the WNGC selects rank 12 which is only two ranks higher than the preferred rank range of 5 to 10.

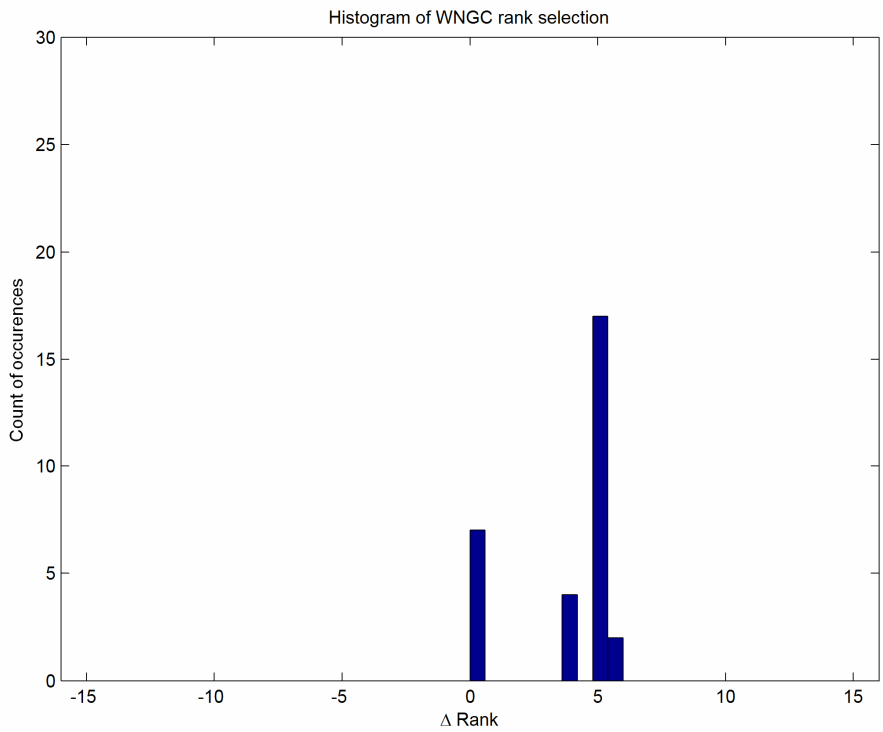

Figure 37: WNGC histogram

Figure 37 shows the histogram of rank selection for a similar example using threshold $\|\mathbf{w}\|^{2}=0.127$. Seven selections out of thirty resulted inside 
the preferred range. The remaining 23 selections are an average of five ranks above the preferred range. Though this shows that we pay some extra processing cost, it is not a serious concern because the selected ranks are well within reason economically.

What is a serious concern is the high variance of the preferred WNGC threshold in Table 5, which means that our average threshold of 0.127 cannot be universally applied.
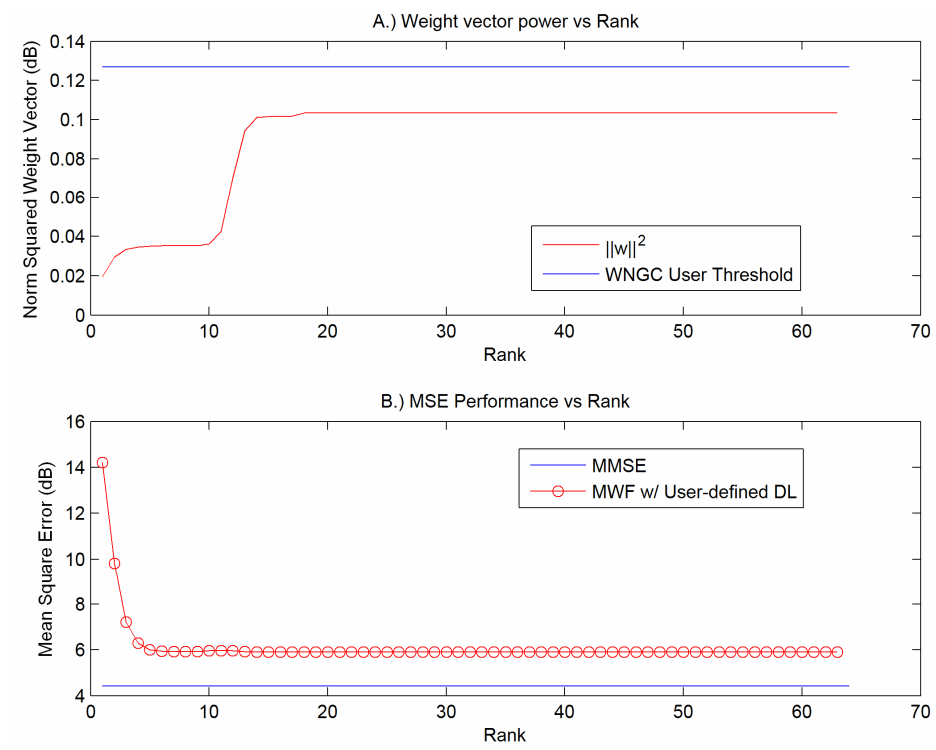

Figure 38: Example of WNGC over thresholding

Figure 38 shows an example where the threshold of 0.127 overshoots the entire $\|\mathbf{w}\|^{2}$ curve, which results to a full rank process. Figure 39 shows the corresponding histogram of 30 trials which reveals that nearly all selections are extreme over-selections. 


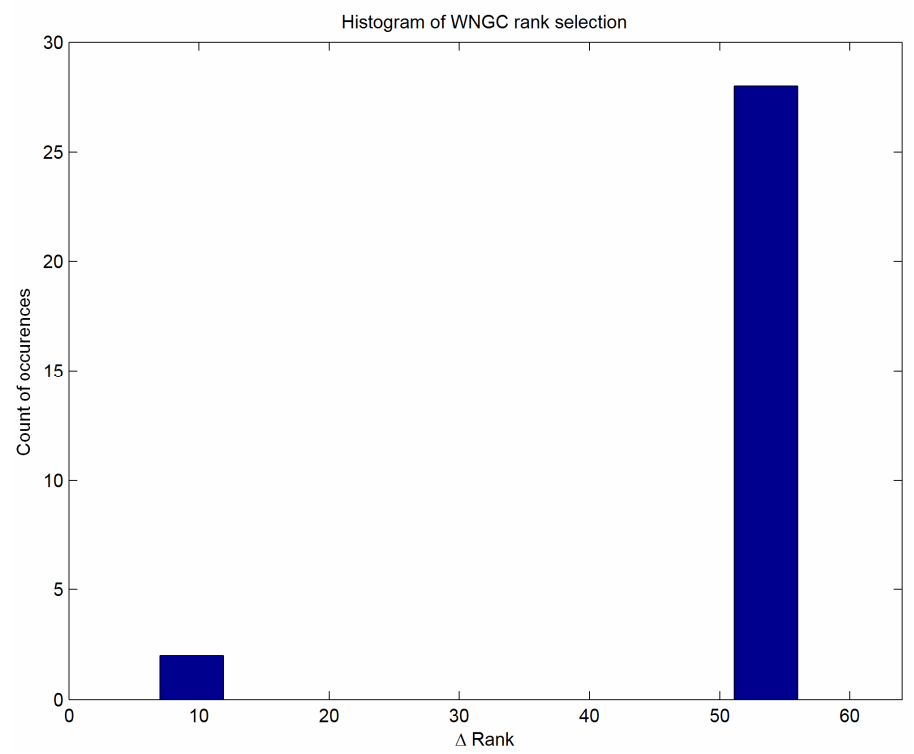

Figure 39: WNGC histogram of over-selections

Results similar to Figure 38 and 39 appeared in the WNGC 100 example study with alarming frequency. This concludes that average WNGC thresholds have too much variance to be practical in unknown environments; however if operator possess a-priori knowledge such as interference power and direction then this technique will yield more useful results.

\subsection{Modified Hanke-Raus error estimation}

The Modified Hanke-Raus error estimation (MHREE) is derived from the original Hanke-Raus error estimate (HREE) stopping algorithm. HREE was developed from conjugate gradient (CG) within the realm of numerical 
linear algebra $(22,21,12)$. In $(65)$ it is shown that the method of conjugate gradients can be used to implement the MWF, therefore techniques developed in the context of CG could be used in the MWF as well. MHREE is one such example.

\subsubsection{The Hanke-Raus error estimation}

Figure 40 shows a filter in its basic form. Input data is $\mathbf{x}, \mathbf{w}$ is weight vector, and $y$ is the output.

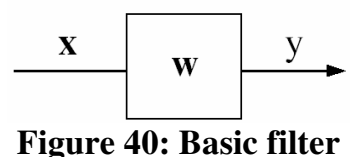

Within linear algebra this translate to

$$
\mathbf{A x}=\mathbf{b}
$$

Equation 6.12

where $\mathbf{A} \in \mathfrak{R}^{M x N}, \mathbf{x} \in \mathfrak{R}^{N}, \mathbf{b} \in \mathfrak{R}^{M}$. In HREE, a bound is established for the error between the exact solution and the computed solution with conjugate gradients (CG), or

$$
\begin{gathered}
\left\|\mathbf{x}_{\text {exact }}-\mathbf{x}_{\mathbf{k}}\right\| \leq \sqrt{P_{\mathbf{k}}(0)}\left\|\mathbf{b}-\mathbf{A} \mathbf{x}_{\mathbf{k}}\right\| \\
\text { Equation 6.13 }
\end{gathered}
$$

where $\mathbf{k}$ is the CG iteration, $P_{\mathbf{k}}$ is a CG polynomial of degree $\mathbf{k}-1$ satisfying the recurrence relation (12) 


$$
P_{\mathbf{k}}(\theta)=\left(1-\theta \alpha_{\mathbf{k}}+\frac{\alpha_{\mathbf{k}} \beta_{\mathbf{k}-1}}{\alpha_{\mathbf{k}-1}}\right) P_{\mathbf{k}-1}(\theta) \frac{\alpha_{\mathbf{k}} \beta_{\mathbf{k}-1}}{\alpha_{\mathbf{k}-1}} P_{\mathbf{k}-2}(\theta)+\alpha_{\mathbf{k}}
$$

Equation 6.14

where $\alpha_{\mathbf{k}}$ and $\beta_{\mathbf{k}}$ are variables used in conjugate gradients least square (CGLS) method. Using CGLS to compute the solution for $\mathbf{x}_{\mathbf{k}}$ yields

$$
\mathbf{x}_{\mathbf{k}}=P_{\mathbf{k}}\left(\mathbf{A}^{T} \mathbf{A}\right) \mathbf{A}^{T} \mathbf{b}
$$

Equation 6.15

Therefore HREE is based on treating the bound in Equation 6.13 as equal and interpret the result as an estimate of error $\left\|\mathbf{x}_{\text {exact }}-\mathbf{x}_{\mathbf{k}}\right\|$. The stopping criteria then select CG iteration (i.e. rank) that minimizes the following function

$$
\mathbf{k}^{\text {hree }}=\frac{\arg \min }{\mathbf{k}} \sqrt{P_{\mathbf{k}}(0)}\left\|\mathbf{b}-\mathbf{A} \mathbf{x}_{\mathbf{k}}\right\|
$$

Equation 6.16 can be translated to the realm of MWF because it is a CG implementation applied to a "normal" equation

$$
\begin{gathered}
\mathbf{A}^{T} \mathbf{A x}=\mathbf{A}^{T} \mathbf{b} \\
\text { Equation } 6.17
\end{gathered}
$$

The least squares solution to $(6.16)$ is known to satisfy this normal equation (29). To draw the connection between Equation 6.16 and the MWF begins with the Wiener-Hopf equation $\mathbf{R}_{\mathbf{x}_{0}} \mathbf{w}_{a}=\mathbf{r}_{\mathbf{x}_{0} d_{0}}$. Recall that

$$
\hat{\mathbf{R}}_{\mathbf{x}_{0}}=\frac{1}{K} \mathbf{X}_{0} \mathbf{X}_{0}^{H}
$$

Equation 6.18 
where $\mathbf{X}_{0}$ is an $\mathbf{N}$ by $K$ matrix and

$$
\hat{\mathbf{r}}_{\mathbf{x}_{0} d_{0}}=\frac{1}{K} \mathbf{X}_{0} \mathbf{d}_{0}^{H}
$$

Equation 6.19

where $\mathbf{d}_{0}$ is a 1 by $K$ vector. Substituting these into Wiener-Hopf equation

results to

$$
\mathbf{X}_{0} \mathbf{X}_{0}^{H} \mathbf{w}_{a}=\mathbf{X}_{0} \mathbf{d}_{0}^{H}
$$

Equation 6.20

Notice the similarity in form between Equation 6.20 and Equation 6.17. If we make a substitution of variables as in Table 6

Table 6: translation of filter paramete
\begin{tabular}{|c|c|c|}
\hline Origina & & $\begin{array}{c}\text { Equivale } \\
\text { I }\end{array}$ \\
\hline A & $---->$ & $\mathbf{X}_{0}^{H}$ \\
\hline $\mathbf{x}$ & $---->$ & $\mathbf{w}_{a}$ \\
\hline b & $---->$ & $\mathbf{d}_{0}^{H}$ \\
\hline
\end{tabular}

we would arrive at the HREE stopping criteria for MWF

$$
r^{\text {hree }}=\frac{\arg \min }{r} \sqrt{P_{r}(0)}\left\|\mathbf{X}_{0}^{H} \mathbf{w}_{a}-\mathbf{d}_{0}^{H}\right\|
$$

which can be reformed to

$$
\begin{gathered}
r^{\text {hree }}=\frac{\arg \min }{r} \sqrt{P_{r}(0)} \sqrt{K * S M S E} \\
\text { Equation 6.22 }
\end{gathered}
$$


where $r$ is the rank index and

$$
S M S E=\frac{1}{K}\left\|\mathbf{X}_{0}^{H} \mathbf{w}_{a}-\mathbf{d}_{0}^{H}\right\|^{2}=\mathbf{w}^{H} \hat{\mathbf{R}}_{\mathbf{x}} \mathbf{w}
$$

Equation 6.23

The term $P_{r}(0)$ is known as the Krylov power function and it is computed as follows

$$
P_{r}(0)=\sum_{i=1}^{r}\left(\theta_{i}^{r}\right)^{-1}
$$

Equation 6.24

The $\theta_{i}^{r}$ is known as the "Ritz values" associated with $r$-stage MWF. These

Ritz values are the eigenvalues of the MWF tri-diagonal $\mathbf{R}_{d}$ matrix (12) which is transformed from the data matrix $\mathbf{R}_{\mathbf{x}}$

$$
\mathbf{R}_{d}=\mathbf{L} \mathbf{R}_{\mathbf{x}} \mathbf{L}^{H}
$$

Equation 6.25

with

$$
\mathbf{L}^{H}=\left[\mathbf{s}, \mathbf{B}_{0}^{H} \mathbf{h}_{1}, \mathbf{B}_{0}^{H} \mathbf{B}_{1}^{H} \mathbf{h}_{2}, \mathbf{B}_{0}^{H} \mathbf{B}_{1}^{H} \mathbf{B}_{2}^{H} \mathbf{h}_{3}, \ldots\right]
$$

Equation 6.26

The original HREE did not perform well in rank selection. The modified HREE can improve this by adding a threshold to the HREE concept. Let

$$
h_{r}=\sqrt{P_{r}(0)} \sqrt{K * S M S E}
$$

and define

Equation 6.27 


$$
h_{r}^{\min }=\min \left\{h_{r}\right\}
$$

Equation 6.28

We define the MHREE stopping criteria to be the max rank such that $h_{r}$ has not significantly exceeded a user-defined minimum

$$
r^{\text {MHREE }}=\max \{r\} \text { subject to } 10 \log h_{r} \leq 10 \log h_{r}^{\min }+\eta^{\text {MHREE }}
$$

where $\eta^{\text {MHREE }}$ is another user-defined threshold.

Figure 41 shows the MWF MSE performance with three jammers at ($76,18,40)$ degrees. The corresponding $h_{r}$ graph details its response for each rank as well as the user threshold $\eta^{\text {MHREE }}$ set at the commonly accepted 5dB. 


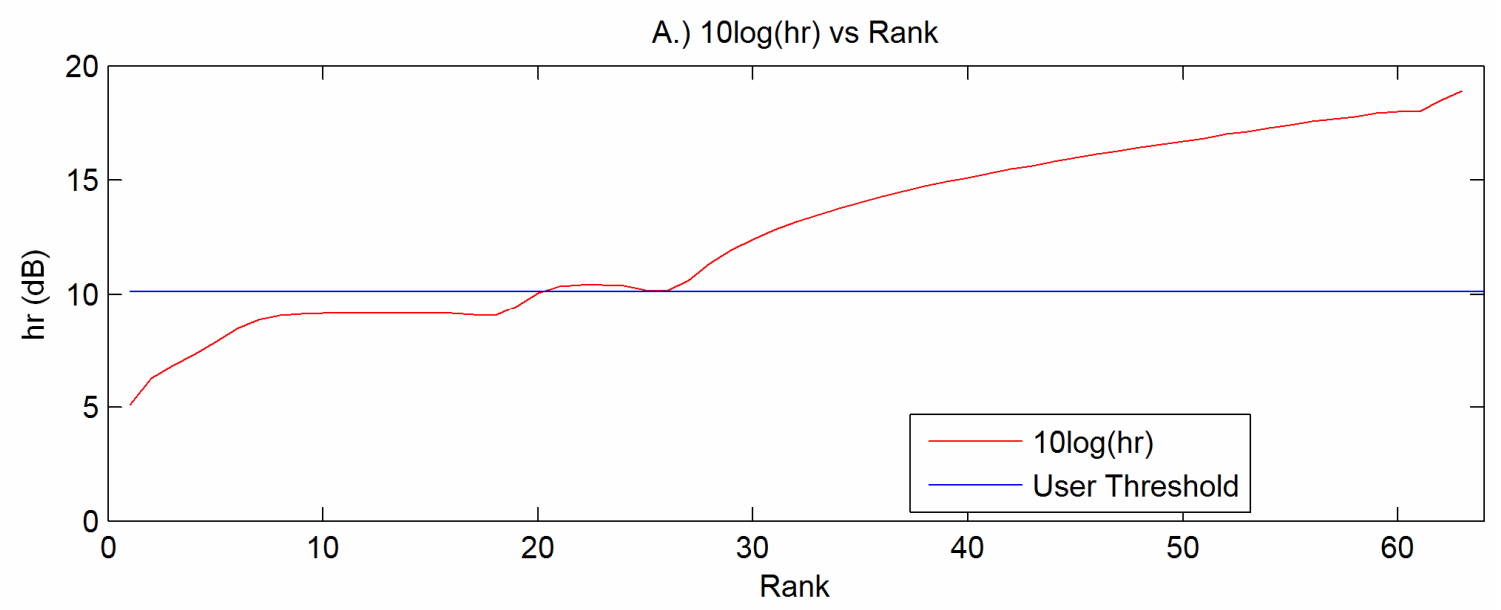

B.) MSE Performance vs Rank

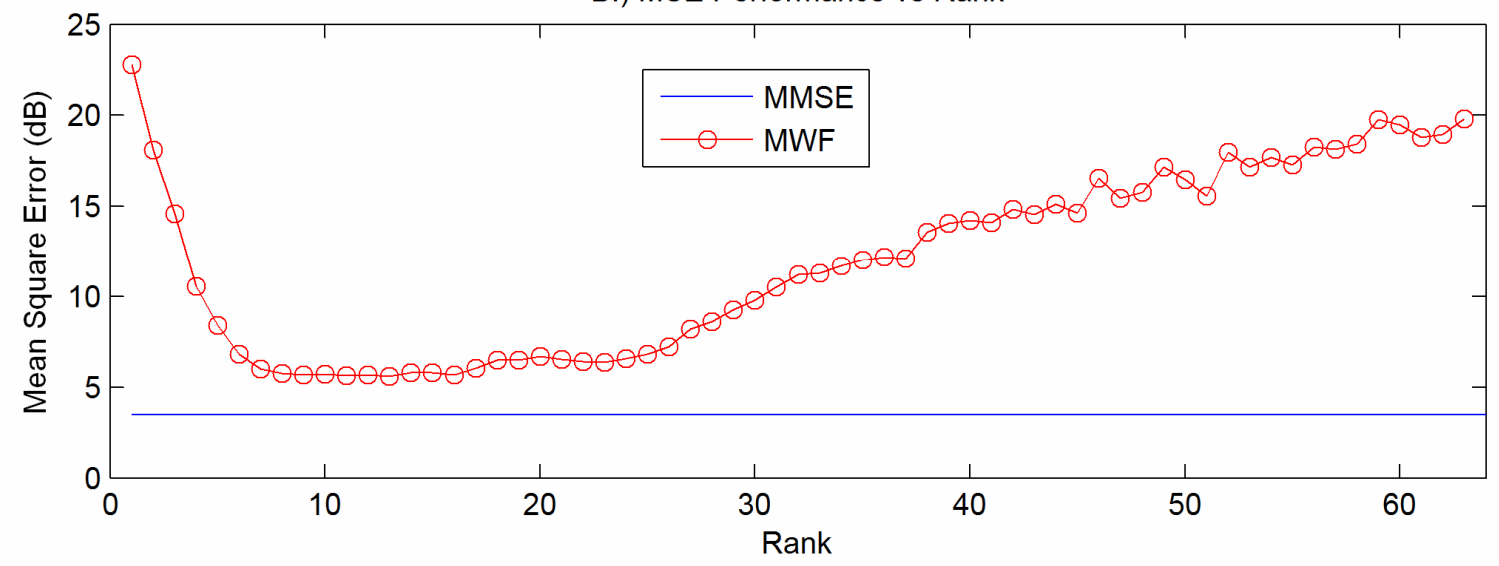

Figure 41: MHREE w/ three jammers

The max rank that satisfies the stopping criteria is 24 , though this rank does provide acceptable MSE performance, its rank selection does not fall within the preferred ranks.

Figure 42 shows another example with four jammers at (-71 -53 87 54) degrees. This time the stopping criteria select rank 33, again the selected rank is outside of the preferred ranks. 


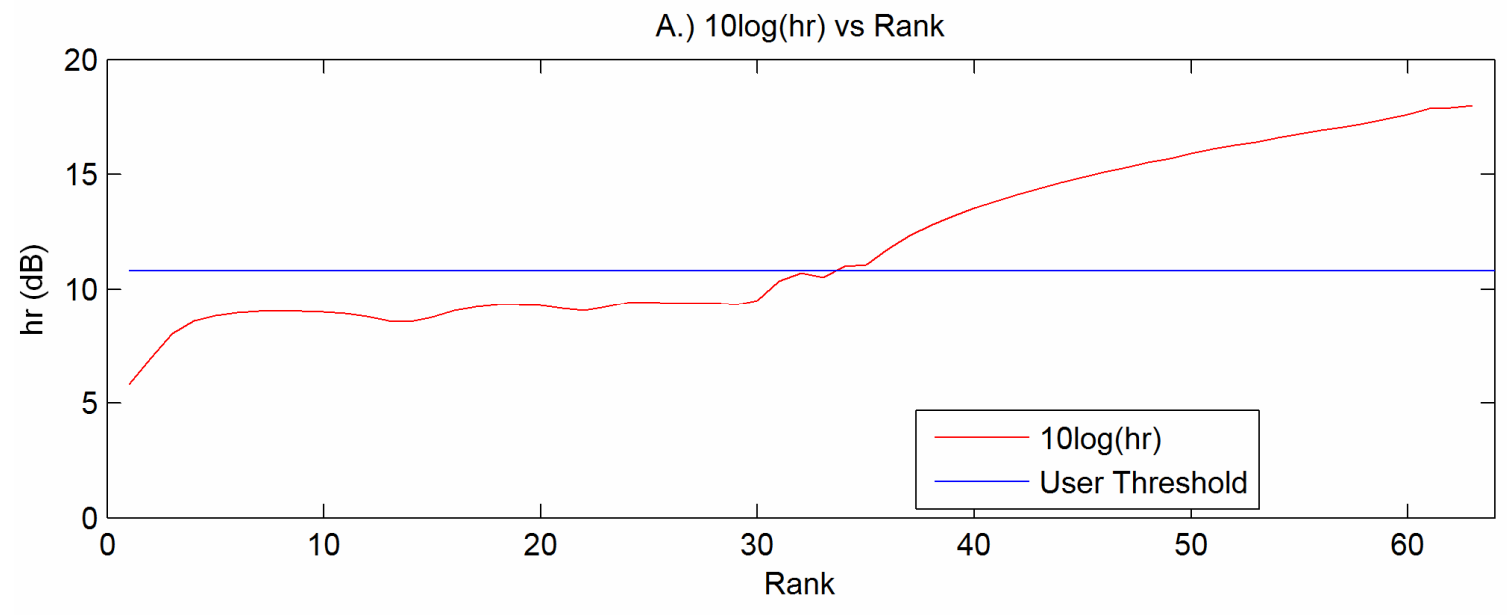

B.) MSE Performance vs Rank

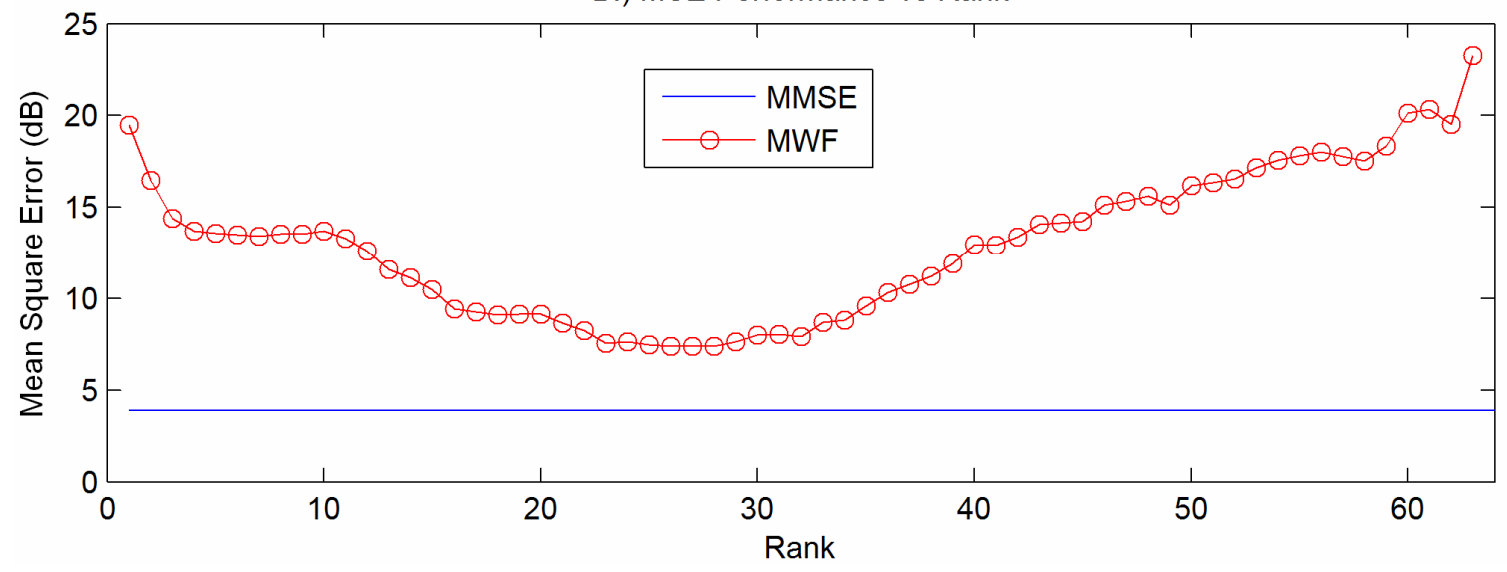

Figure 42: MHREE w/ four jammers

By now you may have come to a conclusion that there is no "one size fits all" threshold. You are correct. Modern day STAP systems utilize multiple sensors, a-priori knowledge and extensive testing experience/data to derive these thresholds to improve decision making. 


\subsection{MHREE with diagonal loading}

Figure 43 shows the MSE performance again with three jammers. This time a diagonal loading (DL) of $10 \mathrm{~dB}$ is included. This will improve MSE performance for ranks pass the optimum rank. User threshold is unchanged. The rank selection (18) is closer to the optimum rank of 14.

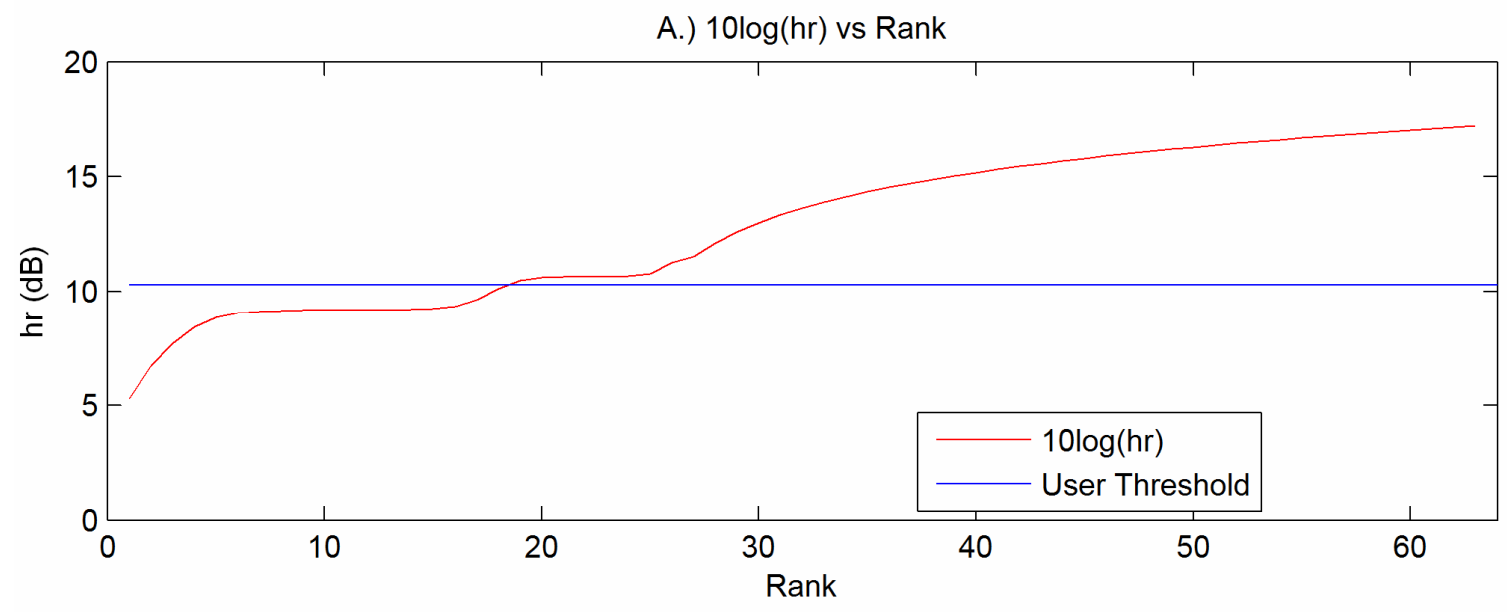

B.) MSE Performance vs Rank

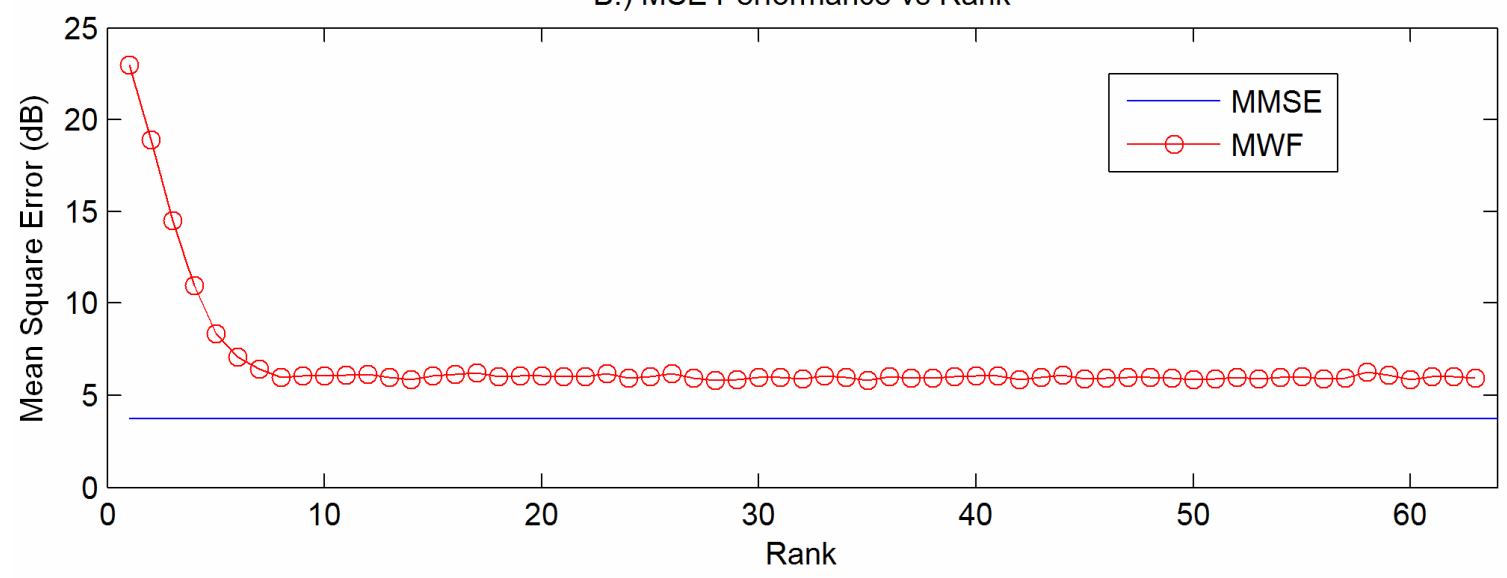

Figure 43: MHREE and diagonal loading w/ three jammers

Figure 44 shows the more difficult situation of four jammers. This time the rank selection is 30 , but improves over its unloaded counterpart. 


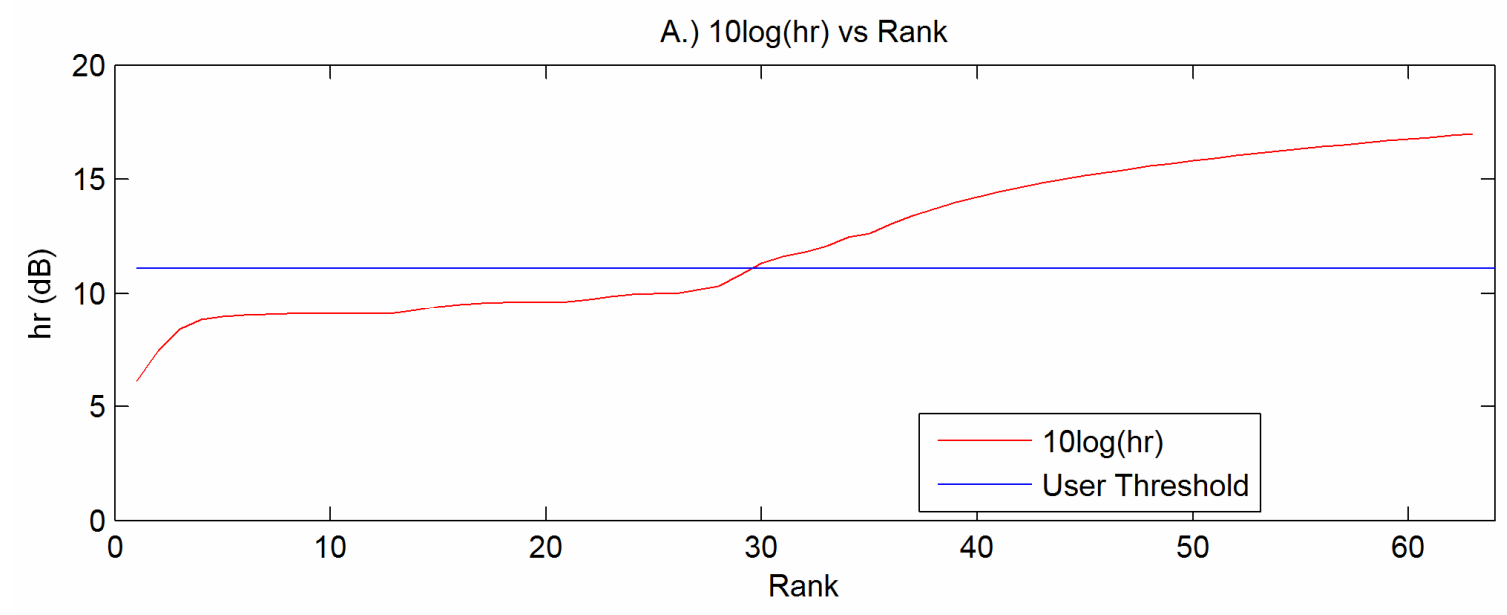

B.) MSE Performance vs Rank

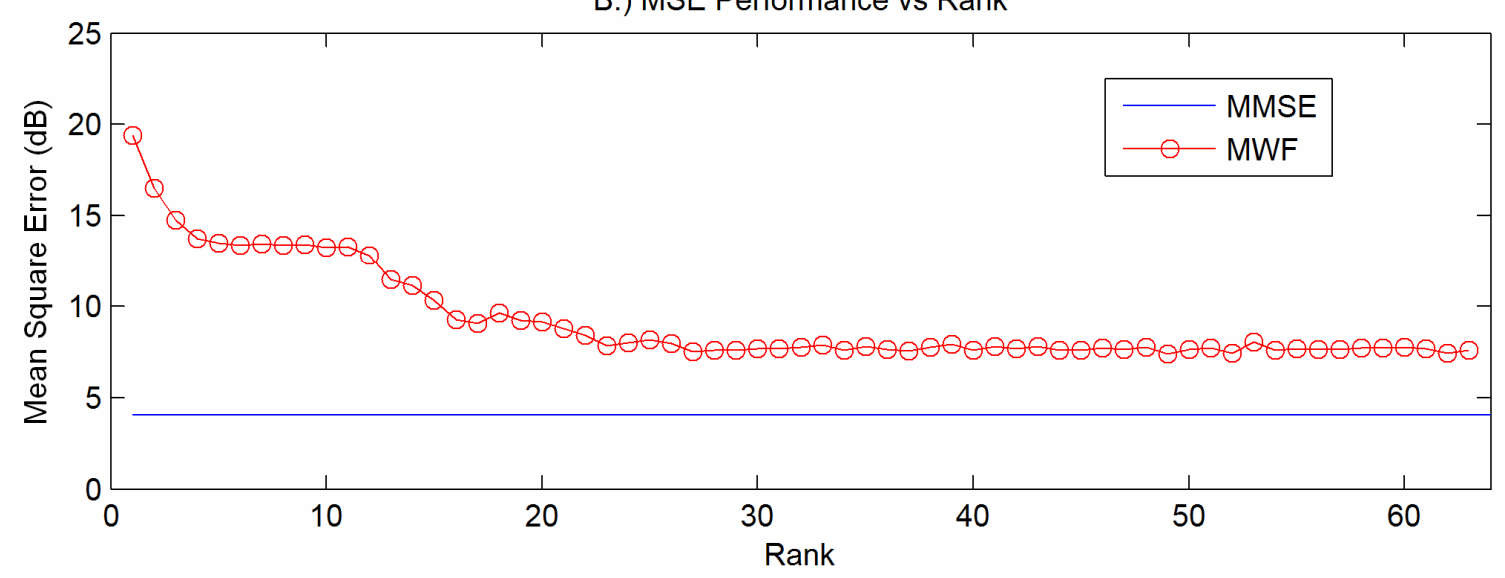

Figure 44: MHREE and diagonal loading w/ four jammers

Above two examples demonstrates that the threshold technique in Equation 6.29 performs well in situations when DL is added, but it does not perform well in all cases. 
Table 7: The preferred $\eta$

\begin{tabular}{|c|c|c|c|c|c|c|}
\hline Jmr 1 Angle & Jmr 2 Angle & Jmr 3 Angle & \begin{tabular}{|} 
Avg Jmr \\
Angle
\end{tabular} & $\begin{array}{c}\text { Minimum } \\
\text { Preferred } \\
\text { Rank }\end{array}$ & $\begin{array}{c}\text { Maximum } \\
\text { Preferred } \\
\text { Rank }\end{array}$ & Preferred $\eta$ \\
\hline-54.908 & 16.566 & 18.457 & 16.396 & 15 & 19 & 4.496 \\
\hline-81.550 & 12.688 & 15.504 & 22.370 & 17 & 21 & 2.702 \\
\hline-43.377 & 31.182 & 35.446 & 29.011 & 13 & 17 & 3.178 \\
\hline-75.930 & 88.584 & 71.522 & 59.615 & 11 & 18 & 3.016 \\
\hline-48.556 & 19.669 & 79.017 & 62.442 & 12 & 19 & 3.175 \\
\hline-53.302 & 67.741 & 47.879 & 63.850 & 10 & 18 & 1.294 \\
\hline-66.875 & 75.232 & 80.321 & 66.310 & 12 & 18 & 2.732 \\
\hline-50.152 & 75.598 & 67.469 & 66.819 & 10 & 16 & 2.933 \\
\hline-21.165 & 76.680 & 71.508 & 67.699 & 11 & 17 & 3.034 \\
\hline-57.390 & 58.877 & 72.304 & 70.910 & 13 & 18 & 2.583 \\
\hline $\begin{array}{l}\text { Average of } \\
\text { Preferred } \eta\end{array}$ & & & & & & 3.081 \\
\hline $\begin{array}{l}\text { Variance of } \\
\text { Preferred } \eta\end{array}$ & & & & & & 0.209 \\
\hline
\end{tabular}

Table 7 shows 10 examples from a 50 example study on the best $\eta^{\text {MHREE }}$ value. Like the previous study with WNGC thresholds, the variance of $\eta^{\text {MHREE }}$ for the preferred ranks is also noticeably high. But unlike the WNGC study discussed earlier, the average $\eta^{\text {MHREE }}$ threshold of 3.081 never overshot the $h_{r}$ curve and therefore this technique will never result to a time consuming full rank process. This is good news, but the performance of these rank selections is a slightly different story. 

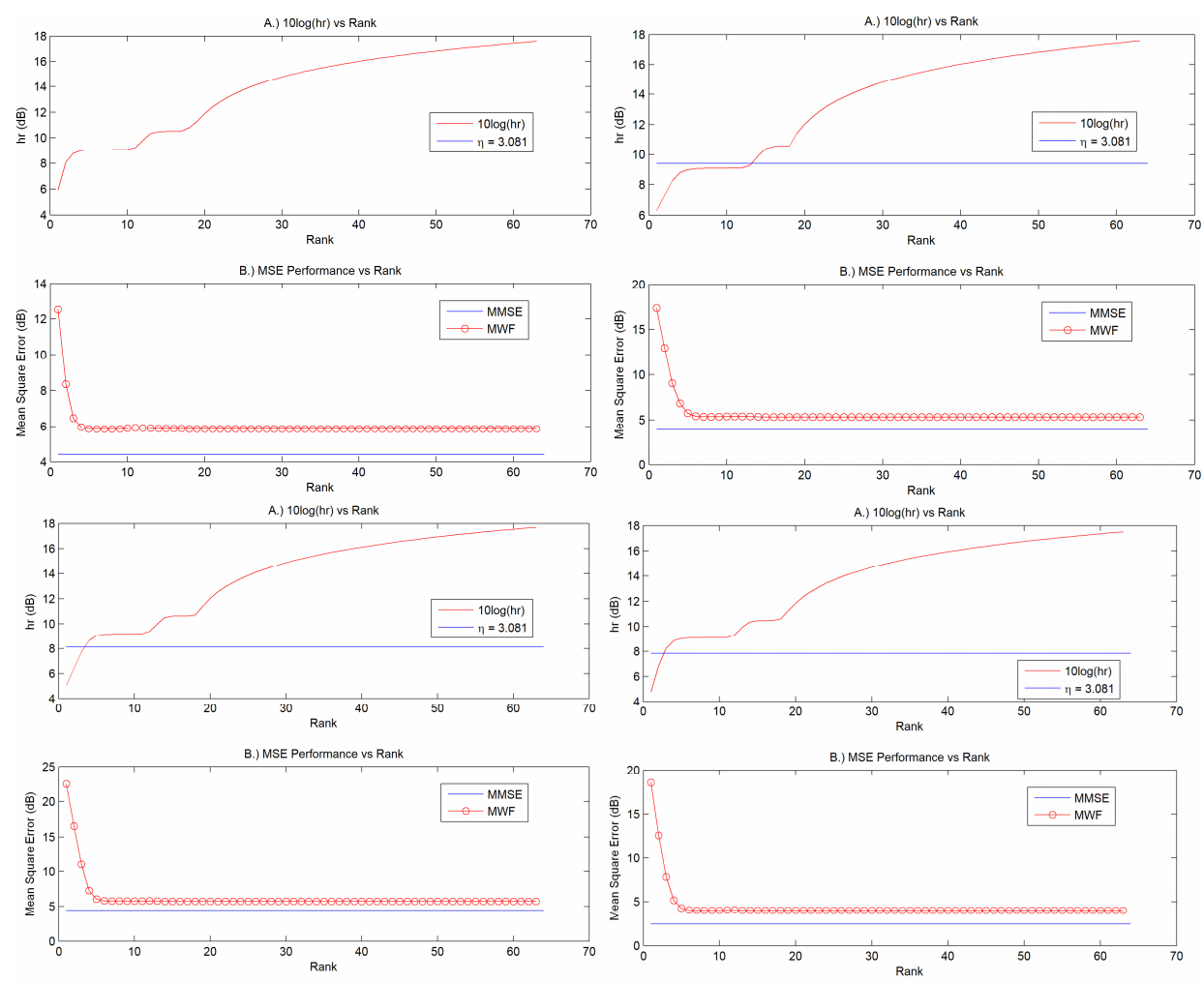

Figure 45: MHREE rank selection with $\boldsymbol{\eta}=\mathbf{3 . 0 8 1}$
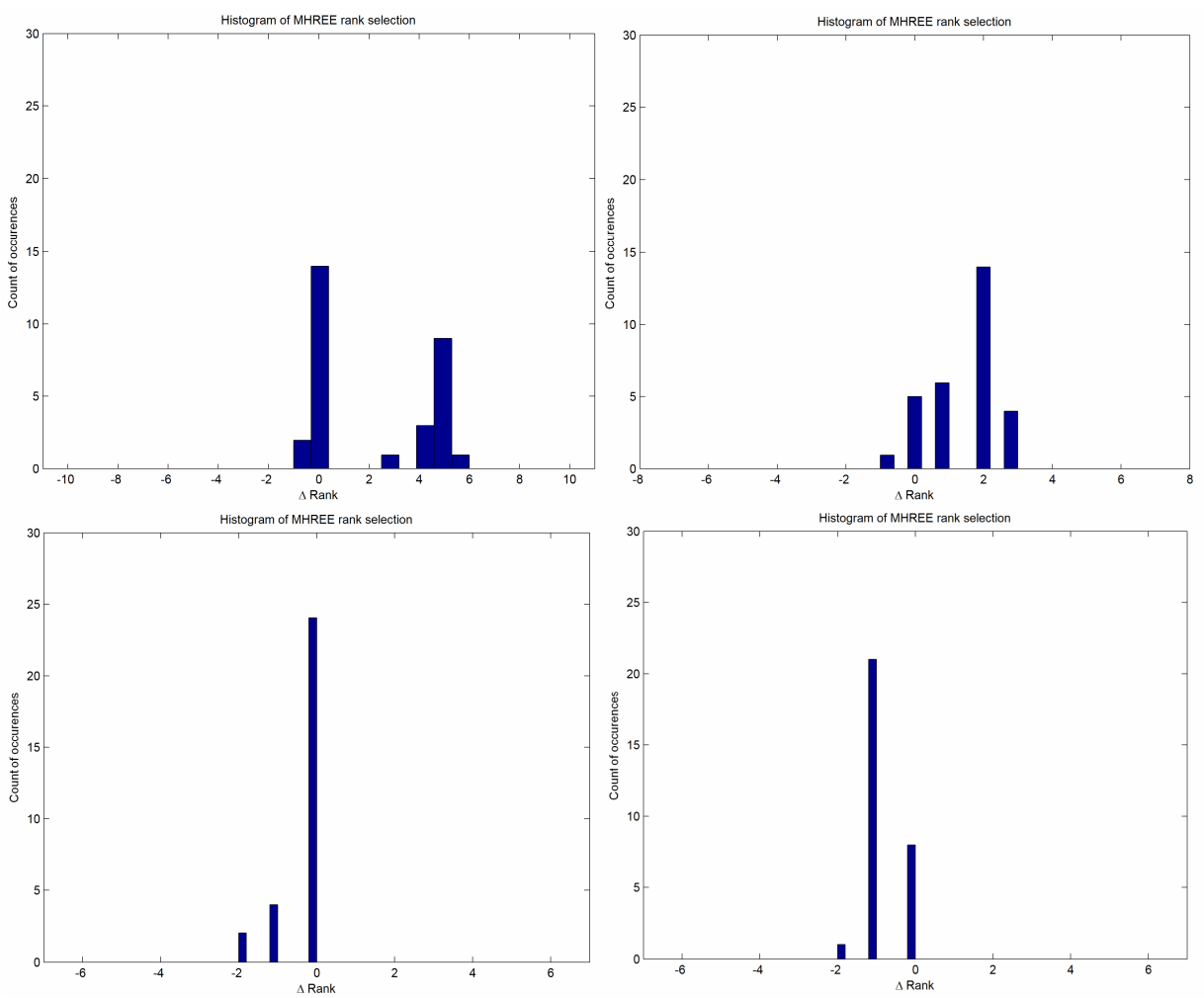

Figure 46: Histogram of MHREE rank selection with $\boldsymbol{\eta}=3.081$ 
Figure 45 and 46 shows four examples of MHREE rank selection and corresponding histogram. They show thirty monte carlos trials using $\eta^{\text {MHREE }}=3.081$. With exception of thirty trials all other simulation parameters are similar to Table 2. These two figures demonstrate this technique's rank selection is generally superior to WNGC but still falls short of the preferred ranks on many occasions. This shortcoming is once again due to the high variance of $\eta^{\text {MHREE }}$ threshold in the preferred ranks.

Another technique for MHREE rank selection is to not use $\eta^{\text {MHREE }}$ threshold but to cap $h_{r}$ directly. Table 8 shows 10 examples from a 50 example study done on this.

Table 8: The preferred $h_{\mathbf{r}}$

\begin{tabular}{|c|c|c|c||c|c|c|}
\hline Jmr 1 Angle & Jmr 2 Angle & Jmr 3 Angle & $\begin{array}{c}\text { Avg Jmr } \\
\text { Angle }\end{array}$ & $\begin{array}{c}\text { Minimum } \\
\text { Preferred } \\
\text { Rank }\end{array}$ & $\begin{array}{c}\text { Maximum } \\
\text { Preferred } \\
\text { Rank }\end{array}$ & $\begin{array}{c}\text { Preferred } \\
\boldsymbol{h} \text { r threshold }\end{array}$ \\
\hline & & & & & & \\
\hline-14.17 & 16.57 & 18.46 & 16.40 & 15 & 19 & 9.537 \\
\hline-38.92 & 12.69 & 15.50 & 22.37 & 17 & 21 & 10.197 \\
\hline-20.41 & 31.18 & 35.45 & 29.01 & 13 & 17 & 9.343 \\
\hline-50.83 & 25.40 & 19.85 & 32.03 & 9 & 13 & 9.179 \\
\hline-47.14 & 45.62 & 19.92 & 37.56 & 6 & 9 & 9.147 \\
\hline-45.82 & 60.39 & 12.56 & 39.59 & 6 & 10 & 9.125 \\
\hline-30.70 & 51.60 & 37.82 & 40.04 & 6 & 9 & 9.119 \\
\hline-74.91 & 33.00 & 17.29 & 41.73 & 5 & 8 & 9.080 \\
\hline \hline-15.37 & 87.82 & 25.36 & 42.85 & 5 & 9 & 9.059 \\
\hline \hline-22.65 & 76.63 & 30.52 & 43.27 & 5 & 10 & 9.135 \\
\hline & & & & & & \\
\hline $\begin{array}{c}\text { Average of } \\
\text { Preferred } \boldsymbol{h} \mathbf{r} \\
\text { Thresholds }\end{array}$ & & & & & & $\mathbf{9 . 1 9 5}$ \\
\hline $\begin{array}{c}\text { Variance of } \\
\text { Preferred } \boldsymbol{h} \mathbf{r} \\
\text { Thresholds }\end{array}$ & & & & & & $\mathbf{0 . 0 0 4}$ \\
\hline
\end{tabular}


Notice this time, the variance of $h_{r}$ threshold is low or 0.004 . This method's rank selection performances are shown in Figure 47 and 48.
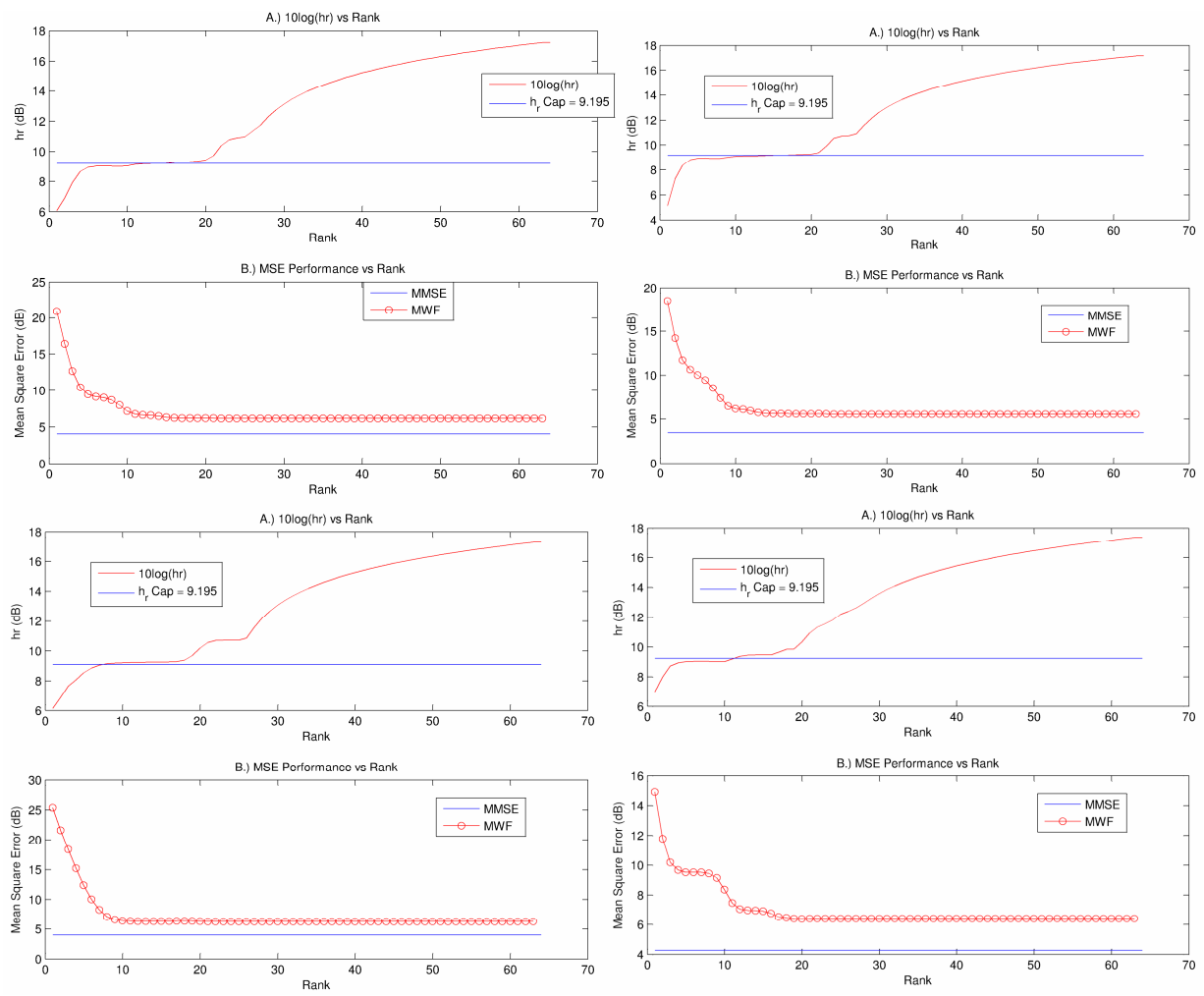

Figure 47: MHREE rank selection with $h r=9.195$ 

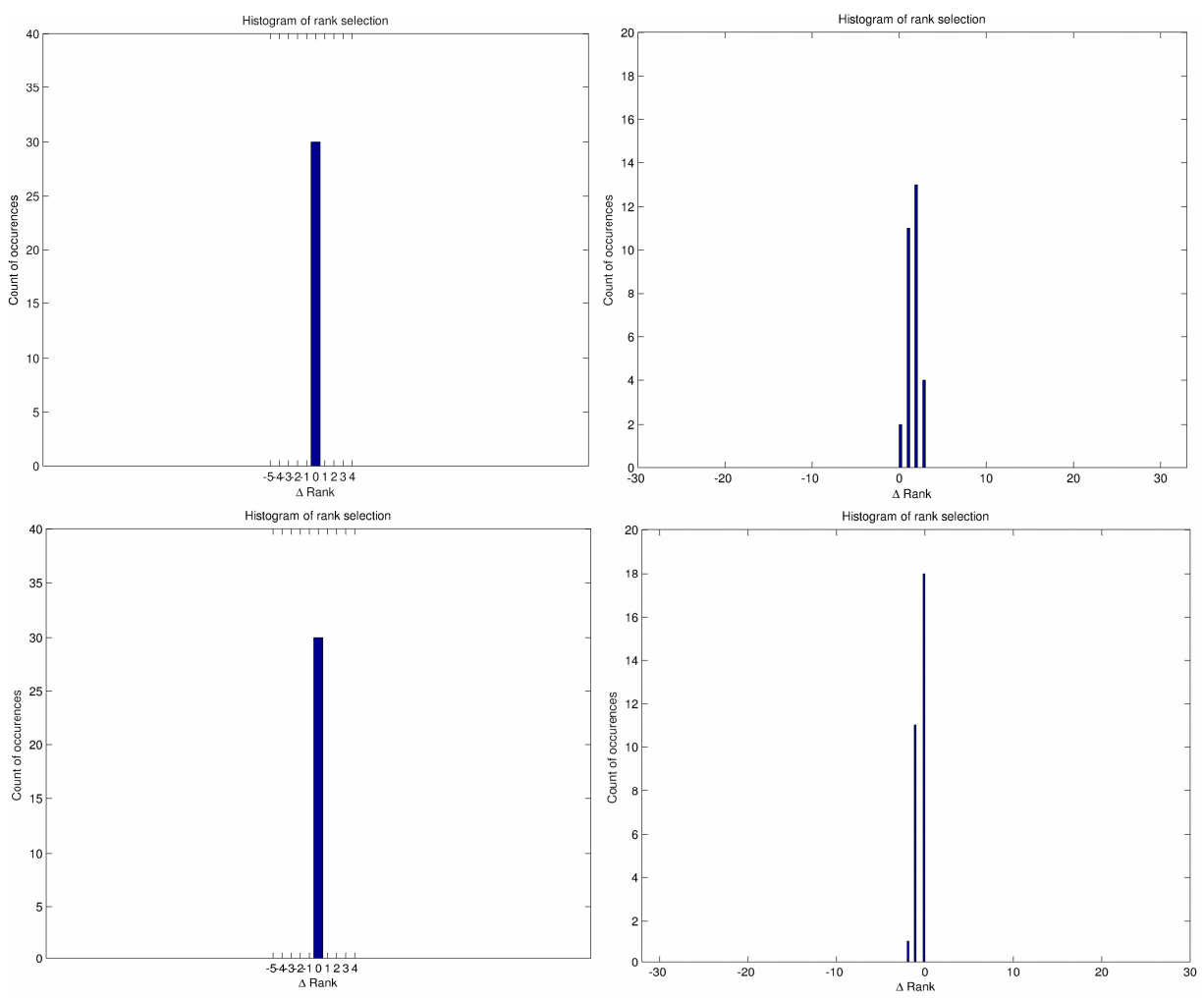

Figure 48: Histogram of MHREE rank selection with $h \mathrm{r}=9.195$

Above two figures demonstrate that by capping $h_{r}$ to 9.195 provides better rank selections than using the technique in Equation 6.29. The low variance of these thresholds certainly made this technique the best performing MWF hard stop method in this thesis.

Table 9 shows the impact of total number of jammers and jammer powers have on the $h_{r}$ threshold. This supports the general consensus that as the number of jammers multiply and jammer power increases, the $h_{r}$ threshold needs to be set higher meaning more adaptive stages are needed to suppress the interference.

Table 9: Best $h \mathrm{r}$ thresholds for various numbers of jammers and jammer powers

\begin{tabular}{|c||c|c|}
\hline \# of Jmrs & JNR = 30 dB & JNR = 50 dB \\
\hline 2 & 8.905 & 9.021 \\
\hline
\end{tabular}




\begin{tabular}{|l||l||c|}
\hline 3 & 8.977 & 9.195 \\
\hline 4 & 9.221 & 9.334 \\
\hline 5 & 9.382 & 9.523 \\
\hline
\end{tabular}

Before we move on it is worth noting that Equation 6.29 can be the more preferable MWF hard stop technique when facing completely unknown environments. The $h_{r}$ cap technique shown here can perform better than the technique in Equation 6.29 if we have some a-priori knowledge on the environment such as number of jammers and their strength; however such knowledge on a constantly changing battlefield could be scarce.

This chapter presented performance comparisons for three different hard stop techniques. These examples stress that different scenarios requires different set of approaches. Therefore having a library of multiple stopping algorithms is desirable and provides flexibility to address different applications and environments.

\subsection{Taxonomies of hard stop techniques}

Before this chapter is concluded, we should explore some taxonomy of hard stop algorithms. This will detail some of the choices of hard stop techniques through two categorizations.

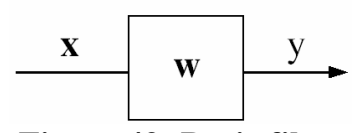

Figure 49: Basic filter 
Please consider once again our basic filter in Figure 49. There are three parameters which can be used to categorize hard stop techniques: the input data $\mathbf{x}$, the weight vector $\mathbf{w}$ and the output $y$. Table 10 shows taxonomy of algorithms based on these criterions. Several of these are well known in the field.

Table 10: Taxonomy of "hard stop" techniques based on filter parameters

\begin{tabular}{|c|c|c|c|}
\hline $\begin{array}{c}\text { A.) Analyze input } \\
\text { data } \mathbf{x}\end{array}$ & $\begin{array}{c}\text { B.) Analyze filter weight } \\
\text { w }\end{array}$ & $\begin{array}{c}\text { C.) Analyze } \\
\text { output } \mathbf{y}\end{array}$ & D.) Hybrid techniques \\
\hline Eigenvalue & Limiting the size of & Based on \\
observable & L-Curve is a technique \\
Decomposition & such as WNGC & $\begin{array}{c}\text { that depends on both } \\
\text { such as Sample }\end{array}$ & SMSE and norm of \\
& adaptive weight
\end{tabular}




\begin{tabular}{|c|c|c|}
\hline & $\begin{array}{l}\text { Mean Square } \\
\text { Error which is } \\
\text { Used in the } \\
\text { Generalized } \\
\text { Discrepancy } \\
\text { Principle (GDP) }\end{array}$ & vector \\
\hline $\begin{array}{l}\text { Find break point } \\
\text { between signal and } \\
\text { noise subspace such } \\
\text { as Akaike Information } \\
\text { Criterion (AIC) }\end{array}$ & $\begin{array}{l}\text { Cross Validation } \\
\text { (CV) is a } \\
\text { method that } \\
\text { depend on } \\
\text { Independent } \\
\text { Sample Mean } \\
\text { Square Error } \\
\text { (ISMSE) }\end{array}$ & $\begin{array}{c}\text { MHREE combines use } \\
\text { both SMSE and Kyrlov } \\
\text { power function }\end{array}$ \\
\hline $\begin{array}{c}\text { Krylov subspace } \\
\text { analysis such as MWF }\end{array}$ & & $\begin{array}{l}\text { Input-Output Power } \\
\text { Function (IOPF) also } \\
\text { use both SMSE and } \\
\text { Kyrlov power function }\end{array}$ \\
\hline
\end{tabular}

Part A.) describes algorithms that are based on analyzing the input data x. A rank revealing decomposition such as the eigenvalue decomposition (EVD) can be very beneficial. Akaike Information Criterion (AIC) (17) and the Minimum Description Length $(M D L)(61,67)$ are two such examples. Their rank selection techniques apply to the principal components (PC) 
which we already discussed. Other decomposition techniques base on the Krylov subspace also falls into this category.

Part B.) describes techniques based on the weight vector $\mathbf{w}$. White noise gain constraint (WNGC) is one such example. Its development was derived from observations that the norm of the weight vector $\|\mathbf{w}\|$ increases in response to mismatch errors (70), therefore resulted in the notion that constraining $\|\mathbf{w}\|$ via a threshold adds robustness.

Part C.) describes algorithms that are based on the output y. The most ideal metric for this would be the mean square error (MSE), however since MSE is unobservable in practice, we have to use something more practical such as sampled mean square error (SMSE). One approach based on the SMSE is the generalized discrepancy principle (GDP) (12). SMSE monotonically decrease with rank and thus cannot truly function as representation of MSE. GDP makes note of this fact by thresholding SMSE for rank selection. Another approach based on output y is the cross validation (CV) algorithm $(20,72)$. This algorithm divides the training data into two partitions, one for training and one for testing. The training and testing samples are independent therefore its SMSE could be more faithful to the true MSE curve. This is also known as the independent sample mean square error (ISMSE) technique (47).

Part D.) describes hybrid algorithms. These use multiple criterions for rank selection. The L-curve $(14,6)$ use both the norm of the adaptive 
weight vector \| w || and SMSE. Other hybrid algorithms include the modified Hanke-Raus error estimate (MHREE) algorithm and the inputoutput power function (IOPF). Like the MHREE, IOPF algorithms also use SMSE and the Krylov power function.

Another insightful categorization of stopping criteria algorithms is their dependency on a threshold. By now you would have encountered the term threshold enough times to make you think that it is analogous to hard stop algorithms. This is partially true but there are algorithms that do not depend on thresholds, we refer to these algorithms as "blind".

Table 11: Taxonomy of "hard stop" techniques based on threshold

\begin{tabular}{|c|c|c|}
\hline Class & With Threshold & Without Threshold \\
\hline Depend on $\mathbf{x}$ & $\begin{array}{c}\text { Eigenvalue } \\
\text { thresholding }\end{array}$ & AIC/MDL \\
\hline Depend on $\mathbf{w}$ & WNGC & CV \\
\hline Depend on $\mathbf{y}$ & GDP & L-curve \\
\hline hybrid $\mathbf{w y}$ & & IOPF \\
\hline hybrid $\mathbf{x y}$ & MHREE & \\
\hline
\end{tabular}

Table 11 shows this categorization. The four that does require a threshold are in the middle. Eigenvalue thresholding requires that we know the threshold level separating the signal and noise eigenvalues 
(preferably from prior knowledge); WNGC needs a threshold on $\|\mathbf{w}\|^{2}$; GDP requires a cap on SMSE; and MHREE needs a threshold on its error estimate function.

The four of the right most columns are "blind". They typically rely on a minimizing a function. AIC and MDL each have embedded penalty functions that forces a minimum for rank selection. CV also generates a minimum for its ISMSE. The L-curve is based upon finding the "knee" of a curve that correlates adaptive weights $\mathbf{w}_{a}$ and SMSE.

It is the hope of this author that these categorizations can help future system designers to understand the intricacies of different approaches so one might articulate a wide variety of potential stopping criteria, and perhaps after evaluation, determines the most promising method (or combination of methods) for particular application.

\subsection{Chapter Summary}

In this chapter we described three MWF "hard stop" rank selection techniques and referenced four others. We simulated their performance and found that a rigid user defined threshold yielded respectable but improvable results. 


\section{Chapter 7}

\section{Thesis summary}

This thesis presents a research to advance the understanding and utility of

principle component (PC) and multistage Wiener filter (MWF) for spacetime adaptive processing (STAP). Both are reduced rank adaptive signal processing algorithms that provide many advantages. These include improved sample support (in MWF) to improved performance over their full rank counterpart. We contrasted MWF and PC, and to a less extent cross spectral metric (CSM) and diagonal loading (DL). We focused on adaptive array processing in space-time adaptive processing (STAP).

In Chapter 4 we discussed rank and sample support compressions. We detailed several mechanisms than can contribute to rank compression such as closely spaced signals and filter constraints. We also explained through eigenvector and Krylov basis analysis why the MWF will have greater rank compression than the principal components. Complex environments with many low power interferers are good candidate for MWF rank compression. However, this rank compression did not translate to an equally impressive sample support compression. 
Chapter 5 described a "soft stop" technique called diagonal loading (DL). We discovered that the MWF can saturate at its peak mean square error if the right loading level is applied.

In Chapter 6 we examined "hard stop" algorithms like WNGC and MHREE for MWF rank selection. Multiple simulations and studies are presented to show that one method of using MHREE with DL can yield excellent rank selection performance. However using a combination of these methods from a library of techniques can improve further improve rank selection performance.

Areas for future work include the following: Chapters 5 and 6 only covered a small portion of rank selection techniques for simulation. Plenty of opportunities exist to add other rank selection techniques into the simulation programs in appendices. Their performances can become even more realistic if more interference models are added such as other covariance matrix tapers (CMT) and receiver chain effects like quantization. Additionally, more metrics are needed to assess algorithm performance such as more histogram of rank selection versus the preferred ranks. Lastly, assessing the performance of these algorithms against experimental data would also be an interesting area for future work. 


\section{Bibliography}

(1) J. S. Goldstein and I. S. Reed. Reduced rank adaptive filtering. IEEE Trans. Signal Processing, 45(2):492-496, February 1997.

(2) C. H. Gierull. Statistical analysis of the eigenvector projection method for adaptive spatial filtering of interference. IEE Proc.-Radar, Sonar, Navig., 144(2):57-63, April 1997.

(3) L. J. Grifiths and C. W. Jim. An alternative approach to linearly constrained adaptive beamforming. IEEE Trans. Antennas Propagate, AP30(1):27-34, January 1982.

(4) H. Cox, R. M. Zeskind, and M. M. Owen. Robust adaptive beamforming. IEEE Trans. Acoust., Speech, Signal Processing, ASSP-35(10): 1365-1376, October 1987.

(5) A. M. Haimovich and Y. Bar-Ness. Adaptive antenna arrays using eigenvector methods. In Proc. IEEE ICASSP, pages 980-983, 1988.

(6) P. C. Hansen and D. P. O'Leary. The use of the L-curve in the regularization of discrete ill-posed problems. SIAM J. Sci. Comput., 14(6): 1487-1503, November 1993. 
(7) C. Eckart and $G$. Young. The approximation of one matrix by another of lower rank. Psychometrika, 1:211-218, 1936.

(8) B. Friedlander. A signal subspace method for adaptive interference cancellation.

IEEE Trans. Acoust., Speech, Signal Processing, 36:1835-1845, December 1988.

(9) L. E. Brennan and I. S. Reed. Theory of adaptive radar. IEEE Trans.

Aerosp. Electron. Syst., 9:237-251, March 1973.

(10) W. L. Brogan. Modern Control Theory. Prentice-Hall, Englewood Cliffs, NJ, third edition, 1991.

(11) B. D. Carlson. Covariance matrix estimation errors and diagonal loading in adaptive arrays. IEEE Trans. Trans. Aerosp. Electron. Syst, 24(4):397-401, July 1988.

(12) P. C. Hansen. Rank-Deficient and Discrete III-Posed Problems. SIAM, Philadelphia, PA, 1998.

(13) G. H. Golub and C. F. Van Loan. Matrix Computations. Johns Hopkins Univ. Press, Baltimore, MD, third edition, 1996.

(14) P. C. Hansen. Analysis of ill-posed problems by means of the L-curve. SIAM Review, 34(4):561-580, December 1992.

(15) H. Cox and R. Pitre. Robust DMR and multi-rate adaptive beamforming. In Proc. 31 st Asilomar Conf. Signals, Syst. Comput., volume 1, pages 920-924, Pacific Grove, CA, November 1997.

(16) J. S. Goldstein, I. S. Reed, and L. L. Scharf. A multistage representation of the Wiener filter based on orthogonal projections. IEEE Trans. Information Theory, 44(7):2943-2959, November 1998.

(17) H. Akaike. A new look at the statistical model identification. IEEE Trans. Automatic Control, AC-19(6):716-723, December 1974.

(18) J. D. Hiemstra and J. S. Goldstein. Robust rank selection for the multistage Wiener filter. In Proc. IEEE ICASSP, Orlando, FL, May 2002.

(19) J. D. Hiemstra. Colored diagonal loading. In Proc. IEEE Radar Conference, Long Beach, CA, April 2002. 
(20) D. M. Allen. The relationship between variable selection and data augmentation and a method for prediction. Technometrics, 16(1):125-127, February 1974.

(21) M. Hanke. Conjugate Gradient Type Methods for III-Posed Problems. Pitman Research Notes in Mathematics, 327. Longman Scientific \& Technical, Harlow, UK, 1995.

(22) M. Hanke and T. Raus. A general heuristic for choosing the regularization parameter in ill-posed problems. SIAM J. Sci. Comput., 17(4):956-972, July 1996.

(23) M. R. Hestenes and E. Stiefel. Methods of conjugate gradients for solving linear systems. J. Res. Nat. Bur. Stand. , 49:409-436, 1952.

(24) J. R. Guerci. Theory and application of covariance matrix tapers for robust adaptive beamforming. IEEE Trans. Signal Processing , 47(4):977-985, April 1999.

(25) J.R. Guerci. Space-time adaptive processing for radar, Artech House, Norwood, MA, 2003

(26) Richard Klemm. Applications of space-time adaptive processing, IEE Radar, Sonar and Navigation series 14, 2004

(27) Bassem R Mahafza and Atef Z Elsherbeni. Matlab simulations for Radar system design, CRC Press, Boca Raton, FL, 2004

(28) Richard Lyons. Understanding Digital Signal Processing, AddisonWesley publishing, Reading, MA, 1997

(29) A. Bjorck. Numerical Methods for Least Squares Problems. SIAM, Philadelphia, PA, 1996.

(30) E. K. L. Hung and R. M. Turner. A fast beamforming algorithm for large arrays. IEEE Trans. Aerosp. Electron. Syst., AES-19(4):598-607, July 1983.

(31) I. Kirsteins and D. W. Tufts. On the probability density of signal-to-noise ratio in an improved adaptive detector. In Proc. IEEE ICASSP, pages 572575, March 1985.

(32) W. L. Stutzman and G. A. Thiele. Antenna Theory and Design. Wiley, New York, NY, second edition, 1998. 
(33) J. Stewart. Calculus. Brooks/Cole, second edition, 1991.

(34) B. Widrow and S. D. Stearns. Adaptive Signal Processing. Prentice-Hall, Upper Saddle River, NJ, 1985.

(35) P. A. Zulch, J. S. Goldstein, J. R. Guerci, and I.S. Reed. Comparison of reduced-rank signal processing techniques. In Proc. 32nd Asilomar Conf. Signals, Syst. Comput., volume 1, pages 421-425, Pacific Grove, CA, November 1998.

(36) C. Y. Tseng and L. J. Griffiths. A unified approach to the design of linear constraints in minimum variance adaptive beamformers. IEEE Trans. Antenn. Propagat., 40(12): 1533-1542, December 1992.

(37) I. Kirsteins and D. W. Tufts. Adaptive detection using a low rank approximation to a data matrix. IEEE Trans. Aerosp. Electron. Syst., 30(1):55-67, January 1994.

(38) M. D. Zoltowski. Conjugate gradient adaptive filtering with application to space-time processing for wireless digital communications. In Proc. IEEE SAM2002 Workshop, Arlington, VA, August 2002.

(39) S. Haykin. Adaptive Filter Theory. Prentice-Hall, Upper Saddle River, NJ, third edition, 1996.

(40) S. Sud. Reduced Rank Adaptive Filtering Applied to Interference Mitigation in Wideband CDMA Systems. PhD thesis, Virginia Tech, May 2002.

(41) C. Y. Tseng and L. J. Griffiths. A simple algorithm to achieve desired patterns for arbitrary arrays. IEEE Trans. Signal Process. , 40:2737-2746, November 1992.

(42) C. L. Lawson and R. J. Hanson. Solving Least Squares Problems. SIAM, Philadelphia, PA, 1995.

(43) C. L. Liu. Introduction to Combinatorial Mathematics. McGraw-Hill, New York, NY, 1968.

(44) R. J. Mailloux. Covariance matrix augmentation to produce adaptive array pattern troughs. Electronics Letters, 31(10):771\{772, May 1995.

(45) S. L. Marple. Digital Spectral Analysis. Prentice-Hall, Englewood Cliffs, NJ, 1987. 
(46) I. Miller and J. E. Freund. Probability and Statistics for Engineers. Prentice-Hall, Englewood Cliffs, NJ, second edition, 1977.

(47) H. E. Witzgall and J. S. Goldstein. A realizable mean square error estimator applied to rank selection. In Proc. 36th Asilomar Conf. Signals, Syst. Comput., Pacific Grove, CA, November 2002.

(48) D. G. Manolakis, V. K. Ingle, and S. M. Kogon. Statistical and Adaptive Signal Processing. McGraw-Hill, Boston, 2000.

(49) V. A. Morozov. Methods for Solving Incorrectly Posed Problems. Translated by A. B. Aries. Springer-Verlag, New York, NY, 1984.

(50) W. L. Myrick. Low Complexity Anti-jam Processing for GPS via Multistage Nested Wiener Filter. PhD thesis, Purdue University, December, 2000.

(51) N. L. Owsley. Sonar array processing. In S. Haykin, editor, Array Signal Processing. Prentice-Hall, Englewood Cliffs, NJ, 1985.

(52) C. D. Peckhan, A. M. Haimovich, T. F. Ayoub, J. S. Goldstein, and I. S. Reed. Reduced rank STAP performance analysis. IEEE Trans. Aerosp. Electron. Syst., 36(2):664-676, April 2000.

(53) J. D. Hiemstra, M. E. Weippert, H. N. Nguyen, and J. S. Goldstein. Insertion of diagonal loading into the multistage Wiener filter. In Proc. IEEE SAM2002 Workshop, Arlington, VA, August 2002.

(54) M. L. Honig and W. Xiao. Adaptive reduced-rank interference suppression with adaptive rank selection. In Proc. MilComm 2000, volume 2, Los Angeles, CA, October 2000.

(55) M. L. Honig and W. Xiao. Performance of reduced-rank linear interference suppression. IEEE Trans. Information Theory, 47:1928\{1946, July 2001.

(56) D. H. Johnson and D. E. Dudgeon. Array Signal Processing. PrenticeHall, Upper Saddle River, NJ, 1993.

(57) D. C. Ricks, P. G. Cifuentes, and J. S. Goldstein. What is optimal processing for nonstationary data? In Proc. 34th Asilomar Conf. Signals, Syst. Comput., Pacific Grove, CA, November 2000. 
(58) D. C. Ricks, P. G. Cifuentes, and J. S. Goldstein. Adaptive beamforming using the multistage Wiener filter with a soft stop. In Proc. 35th Asilomar Conf. Signals, Syst. Comput., Pacific Grove, CA, November 2001.

(59) D. C. Ricks and J. S. Goldstein. Efficient architectures for implementing adaptive algorithms. In Proc. 2000 Antenna Applications Symposium, Allerton Park, Monticello, IL, September 2000.

(60) D. Ricks. Generating snapshots from a correlation matrix. SAIC Internal Tech Note, March 2000.

(61) J. Rissanen. Modeling by shortest data description. Automatica, 14:465-471, 1978.

(62) J. D. Hiemstra, M. E. Weippert, J. S. Goldstein, and T. Pratt. Application of the L-curve technique to loading level determination in adaptive beamforming. In Proc. 36th Asilomar Conf. Signals, Syst. Comput., Pacific Grove, CA, November 2002.

(63) J. Ward. Space-time adaptive processing for airborne radar. Technical Report 1015, MIT Lincoln Laboratory, December 1994.

(64) M. E. Weippert. The loading lemma. SAIC Internal Tech Note, October 2001.

(65) M. E. Weippert, J. D. Hiemstra, J. S. Goldstein, and M. D. Zoltowski. Insights from the relationship between the multistage Wiener filter and the method of conjugate gradients. In Proc. IEEE SAM2002 Workshop, Arlington, VA, August 2002.

(66) I. S. Reed, J. D. Mallett, and L. E. Brennan. Rapid convergence rate in adaptive arrays. IEEE Trans. Aerosp. Electron. Syst., AES-10(6):853-863, November 1974.

(67) G. Schwarz. Estimating the dimension of a model. Ann. Stat., 6(2):461464, March 1978.

(68) M. I. Skolnik. Introduction to Radar Systems. McGraw-Hill, New York, NY, third edition, 2001.

(69) D. W. Tufts, R. Kumaresan, and I. Kirsteins. Data adaptive signal estimation by singular value decomposition of a data matrix. Proc. IEEE, 70(6):684-685, June 1982. 
(70) H. L. Van Trees. Optimum Array Processing. Wiley, New York, NY, 2002.

(71) J. E. Hudson. Adaptive Array Principles. Peter Peregrinus Ltd., London, 1981.

(72) H. Qian and S. N. Batalama. Data-record-based criteria for the selection of an auxiliary-vector estimator of the MVDR filter. In Proc. 34th Asilomar Conf. Signals, Syst. Comput., volume 1, pages 802-807, Pacific Grove, CA, November 2000.

(73) J. S. Goldstein and I. S. Reed. Subspace selection for partially adaptive sensor array processing. IEEE Trans. Aerosp. Electron. Syst., 33:539-544, April 1997.

(74) D. K. Barton. Modern Radar System Analysis. Artech House, Norwood, MA, 1988.

(75) O. P. Cheremisin. Efficiency of adaptive algorithms with regularized sample covariance matrix. Radio Eng. Electron. Phys. , 27:69-77, 1982.

(76) S. P. Applebaum and D. J. Chapman. Adaptive arrays with main beam constraints. IEEE Trans. Antenn. Propagat., AP-24(5):650-662, September 1976.

(77) J. R. Guerci, J. S. Goldstein, and I. S. Reed. Optimal and adaptive reduced-rank STAP. IEEE Trans. Aerosp. Electron. Syst., 36(2):647-663, April 2000.

(78) J. S. Goldstein, J. R. Guerci, and I. S. Reed. Reduced-rank intelligent signal processing with application to radar. In B. Kosko and S. Haykin, editors, Intelligent Signal Processing, chapter 12. Wiley-IEEE Press, 2001. 


\section{Appedix A: Matlab codes for MWF vs PC-SD MSE performance}

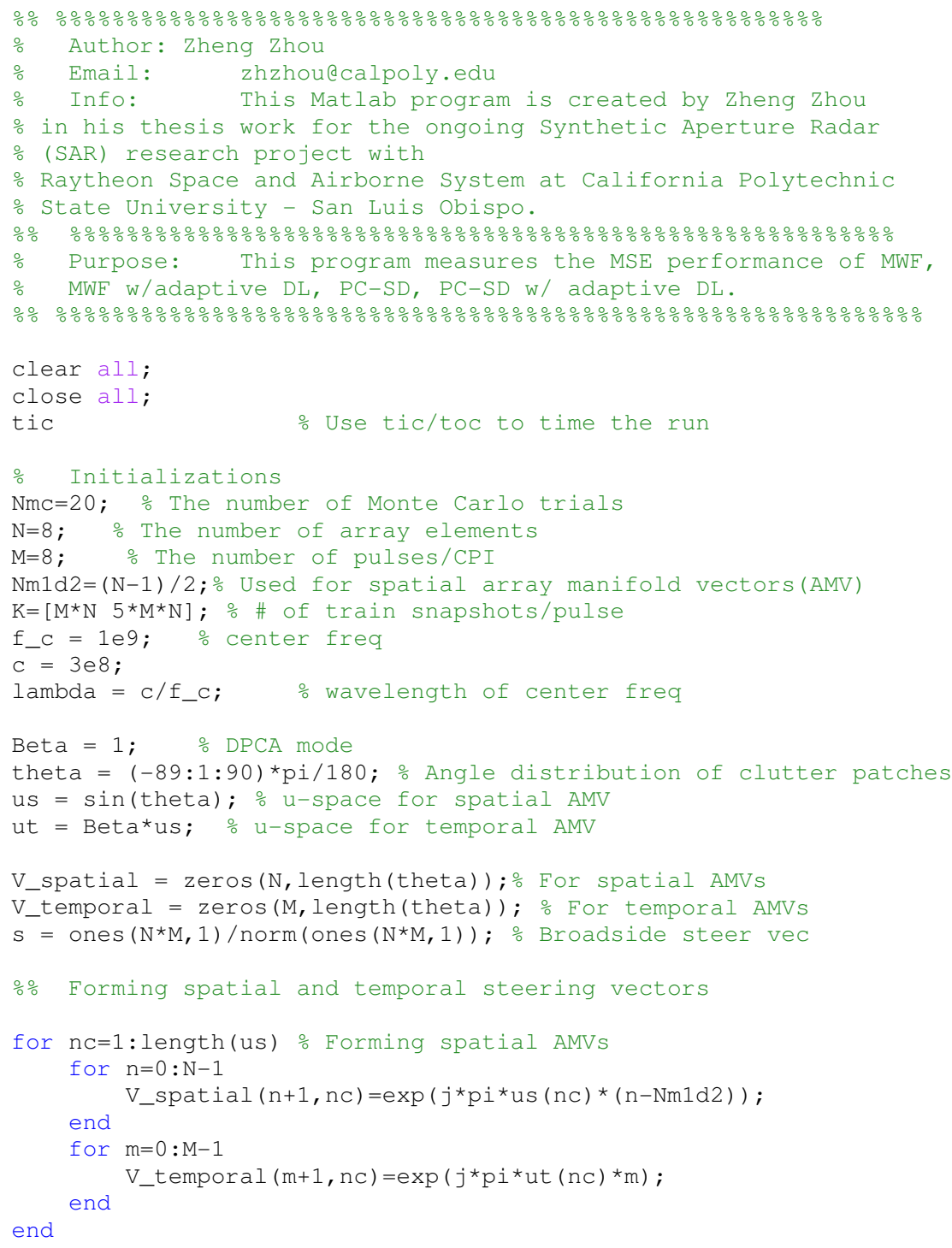




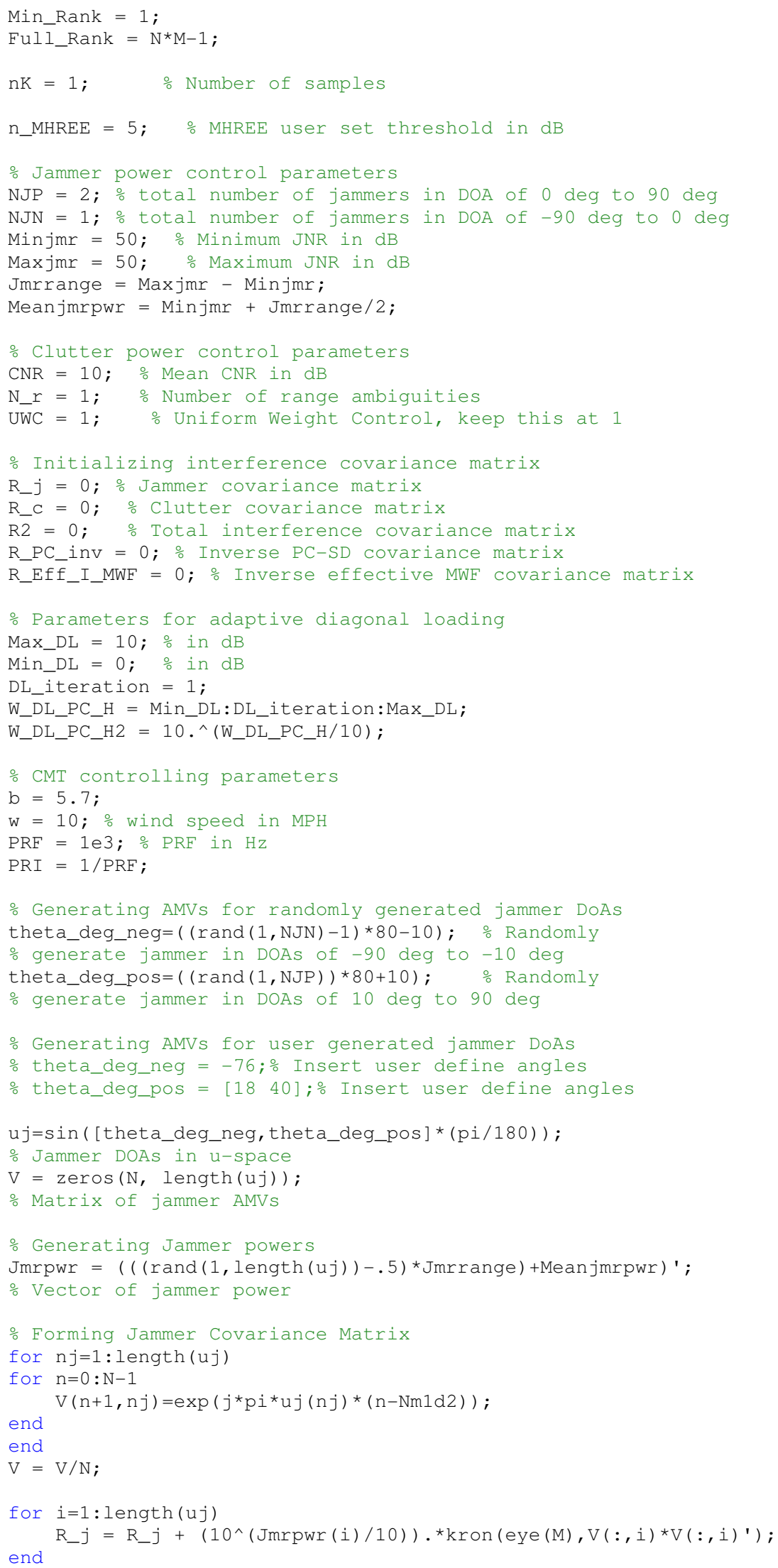




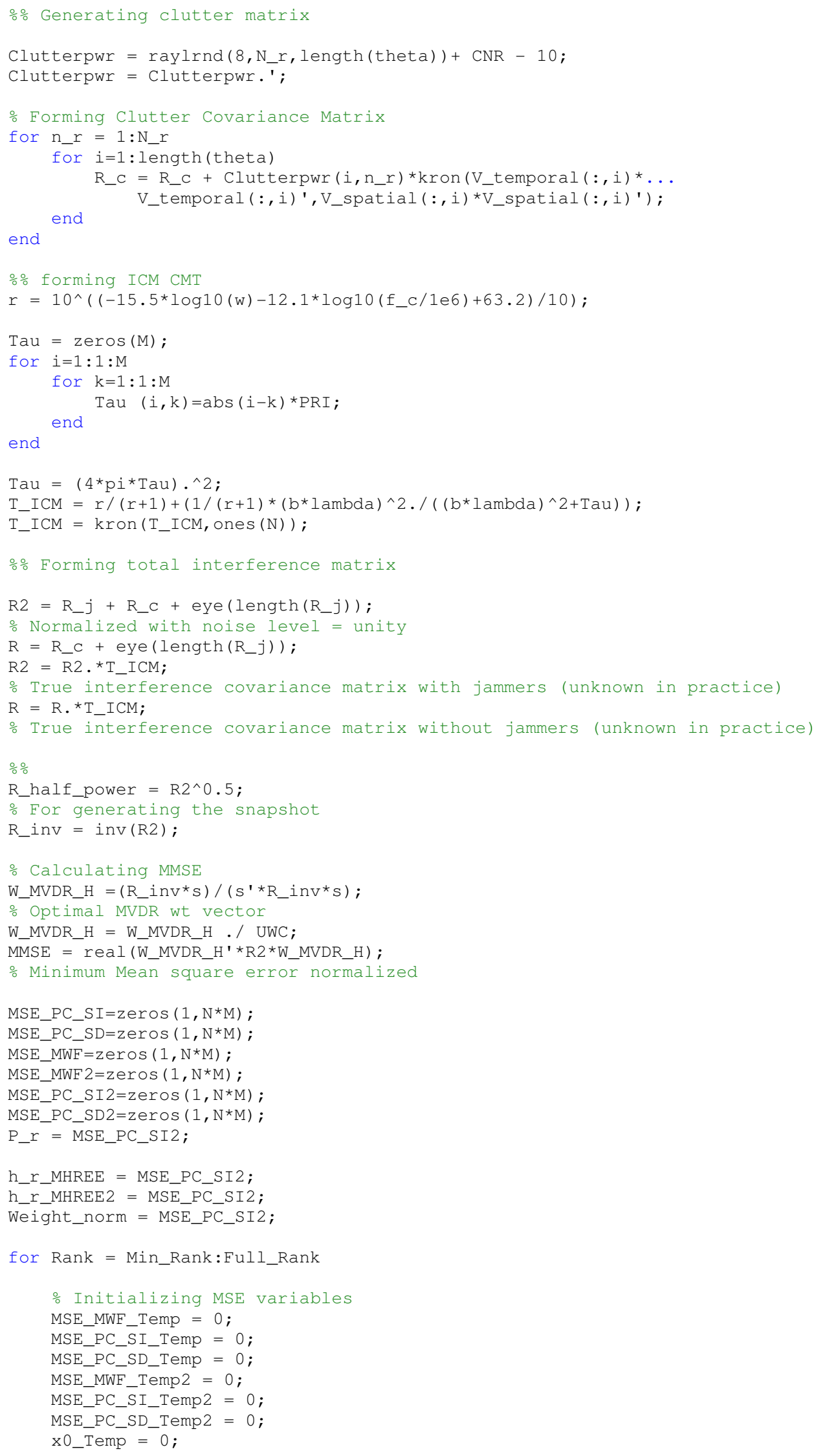




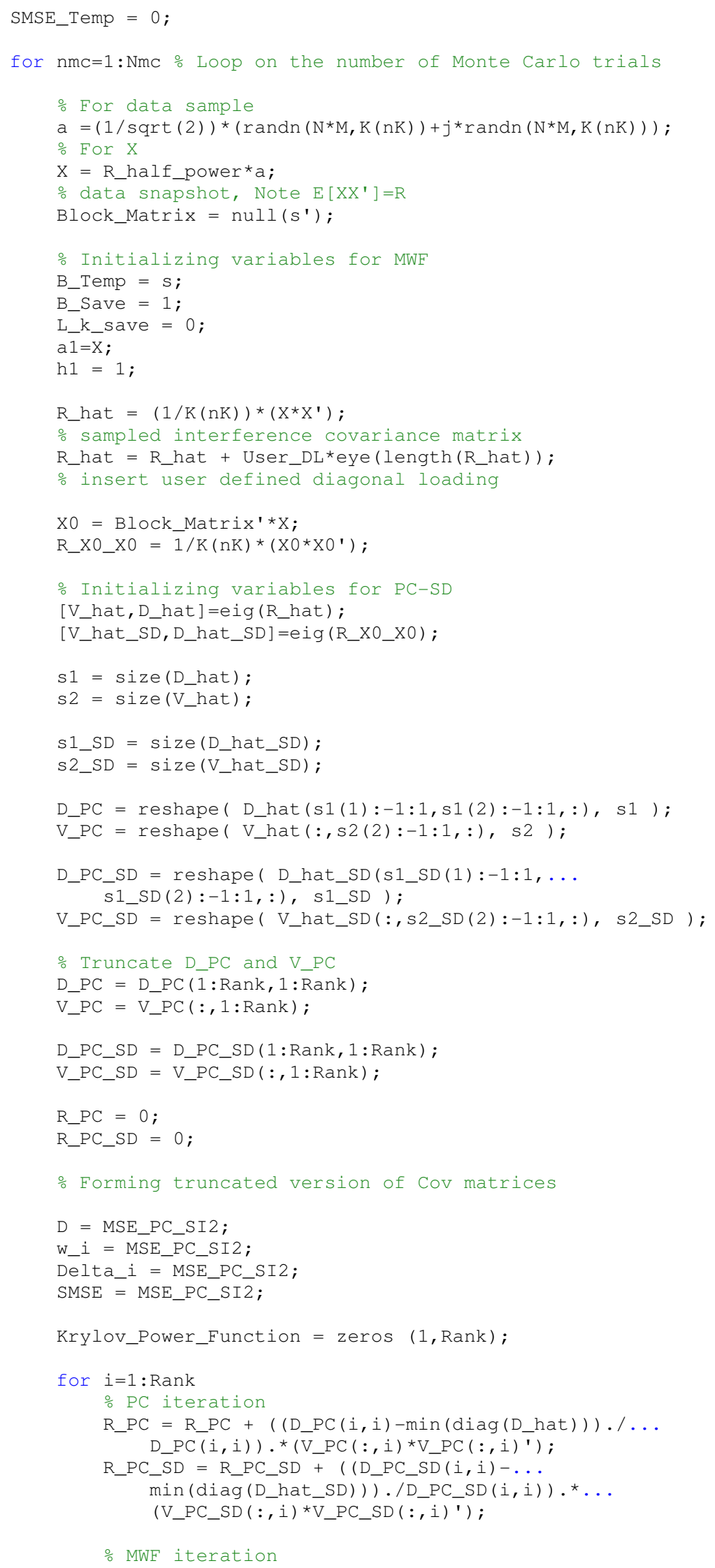




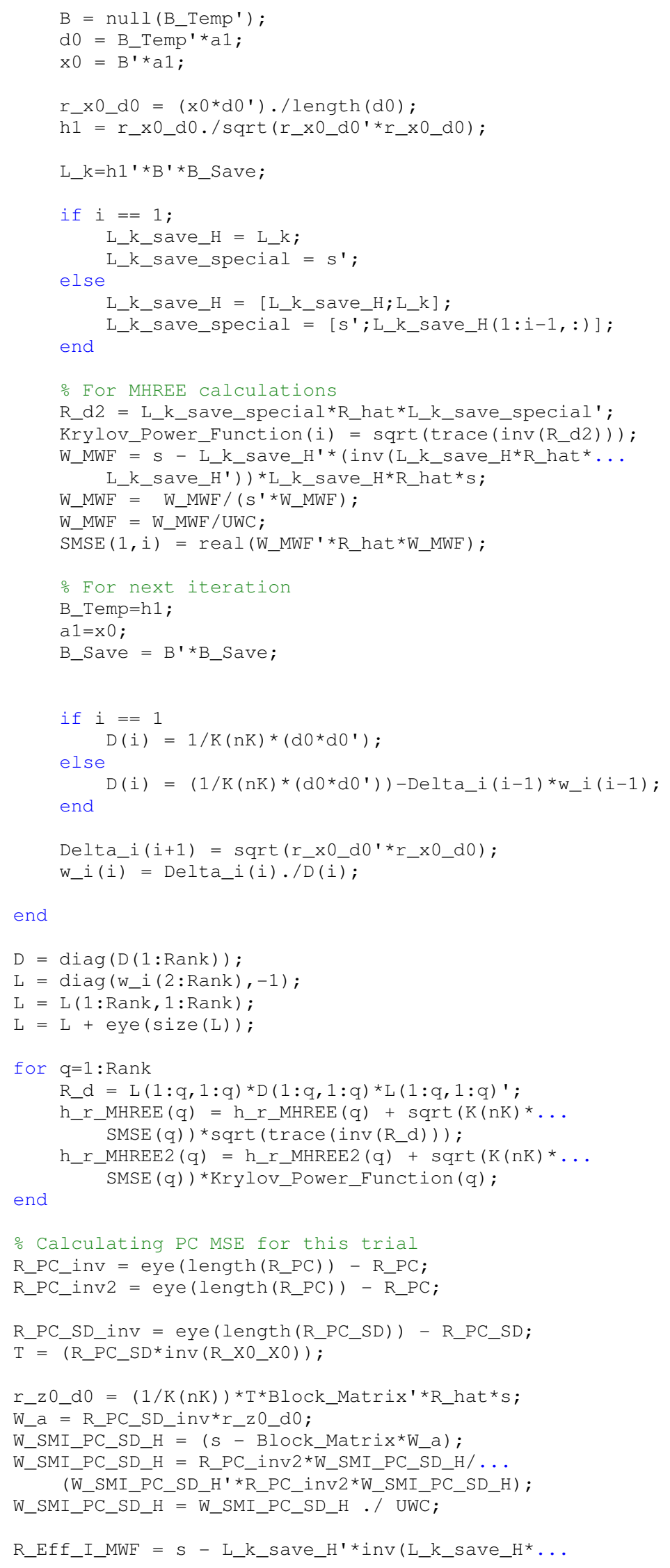




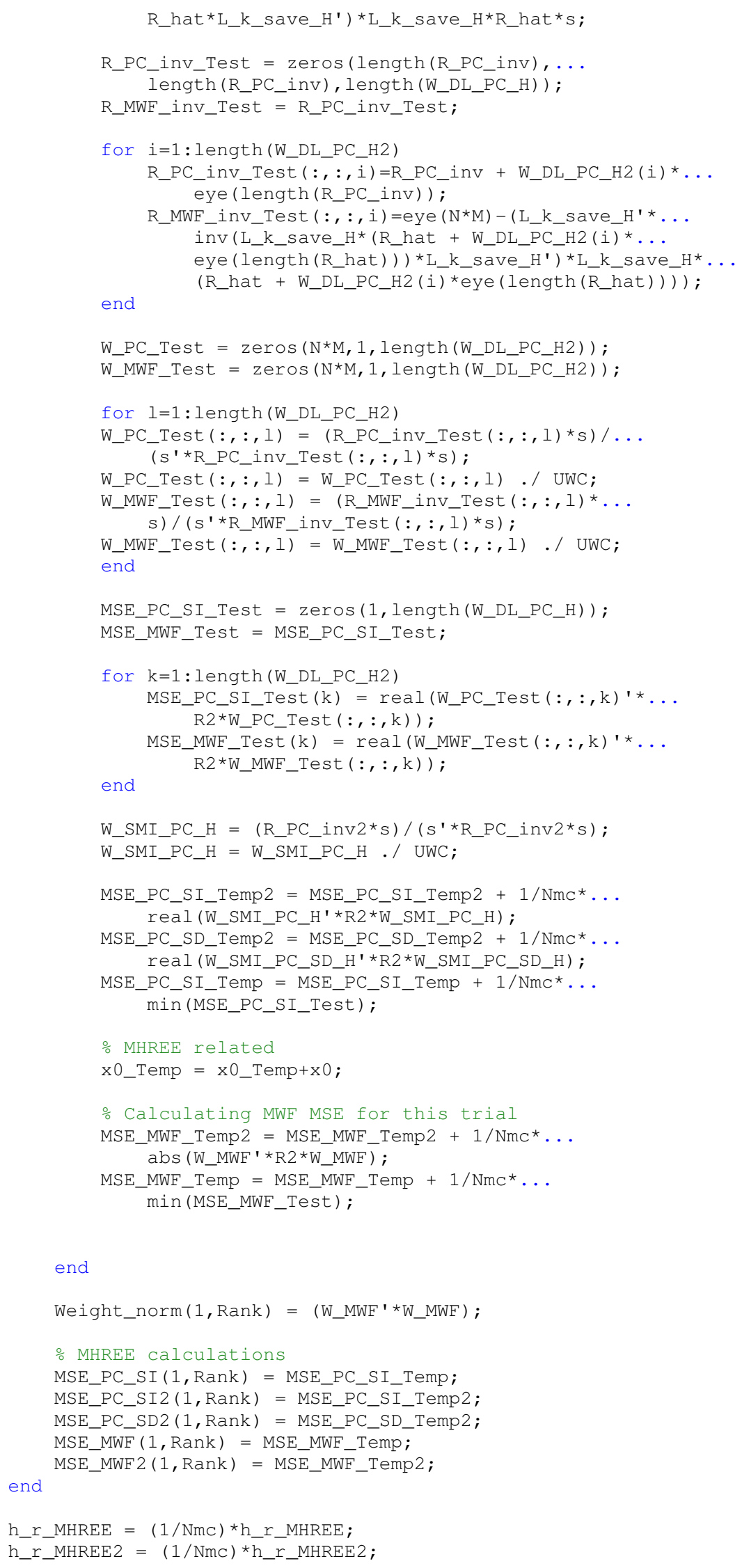




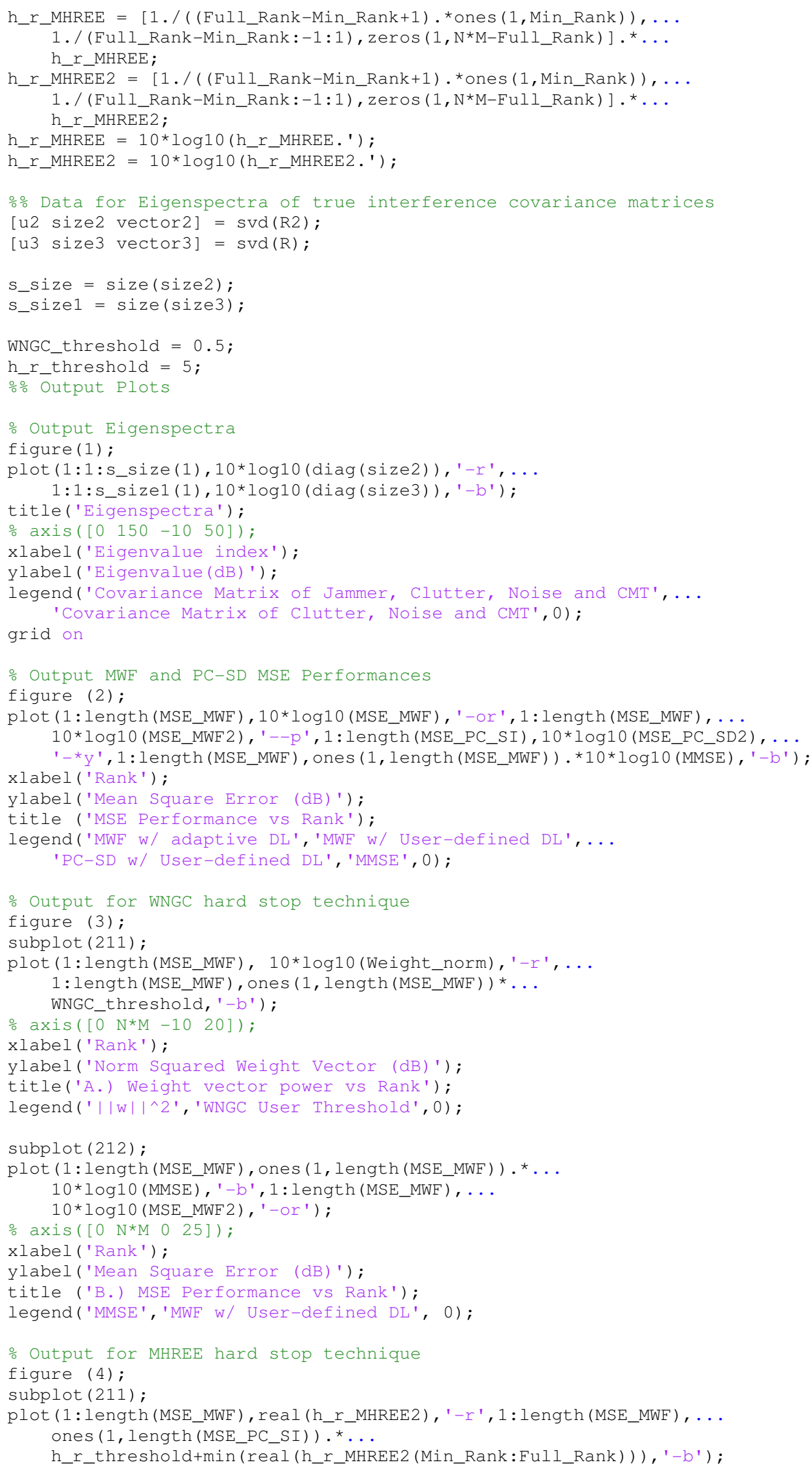




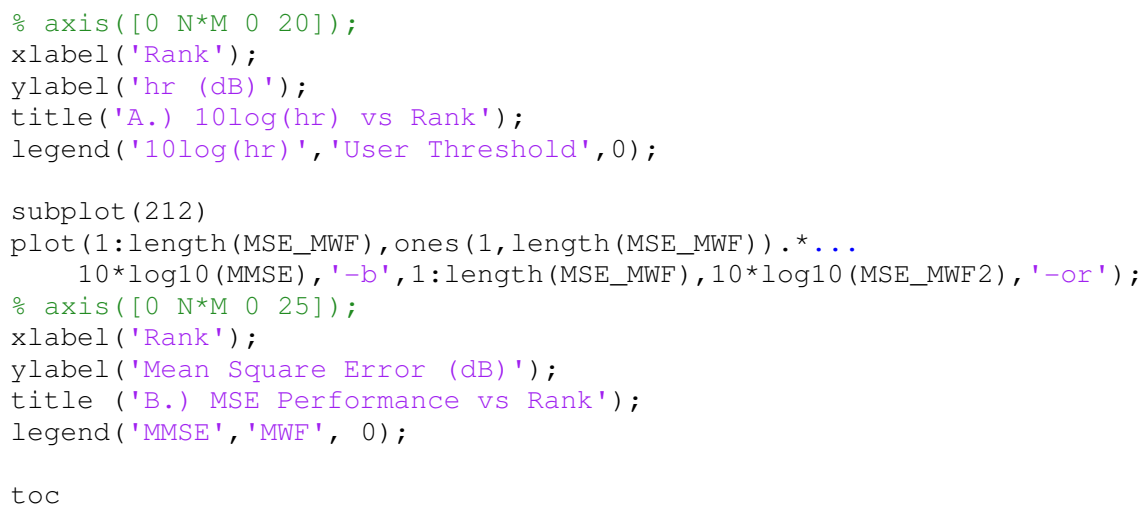




\section{Appedix B: Matlab codes for study of SINR vs sample support}

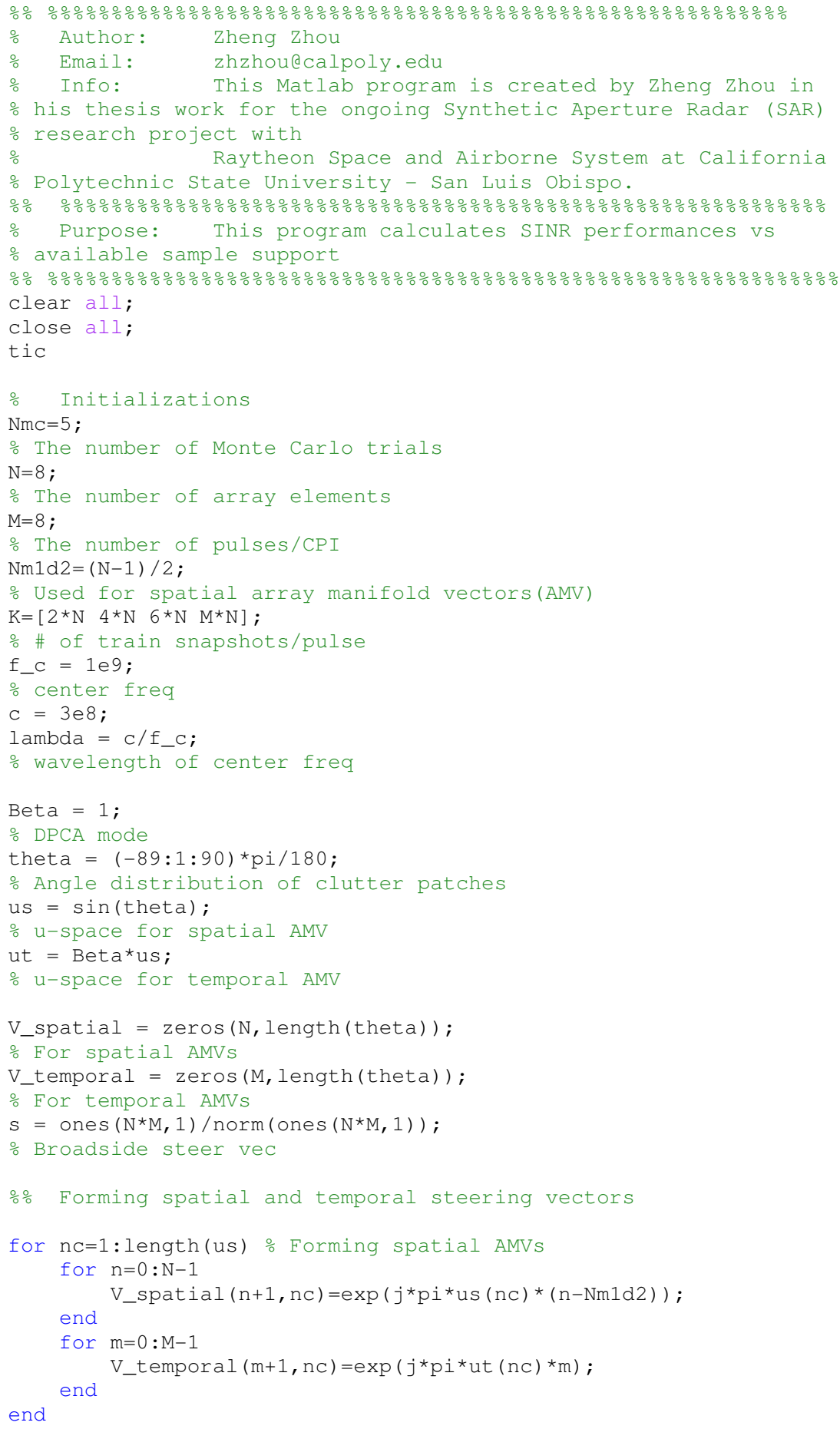

\% Normalize steering vectors 


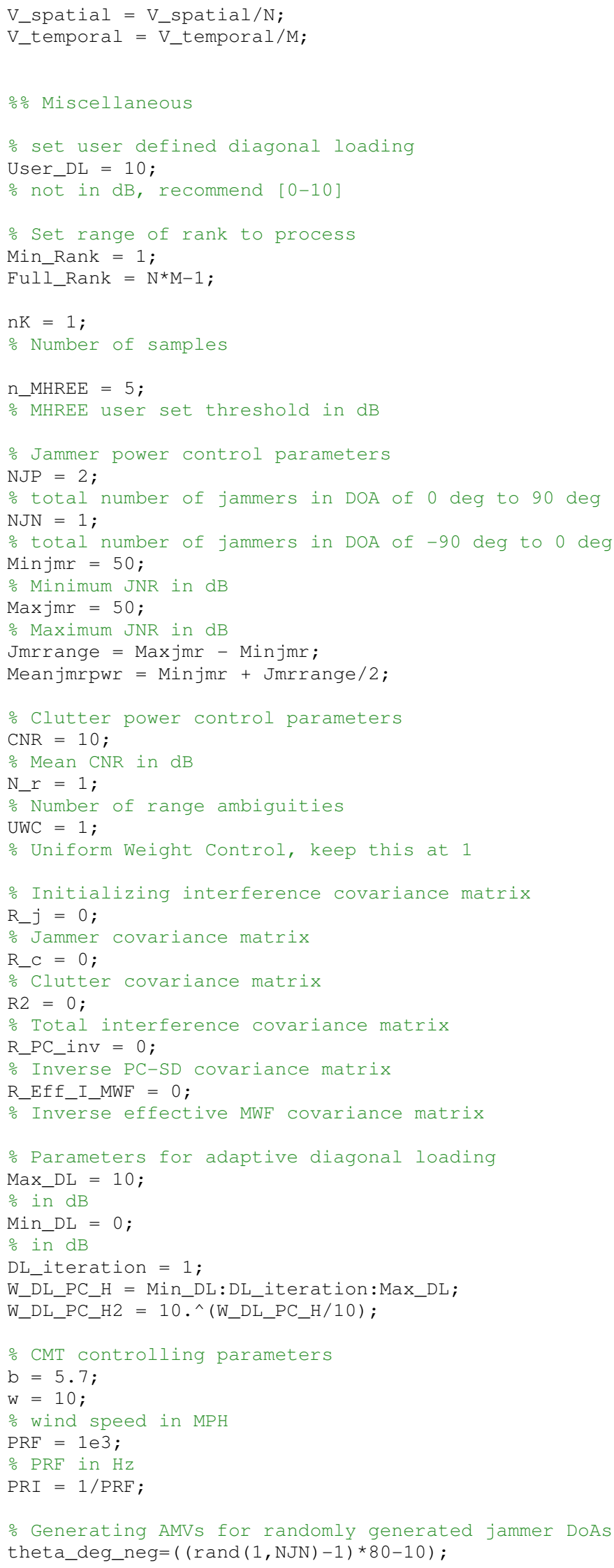




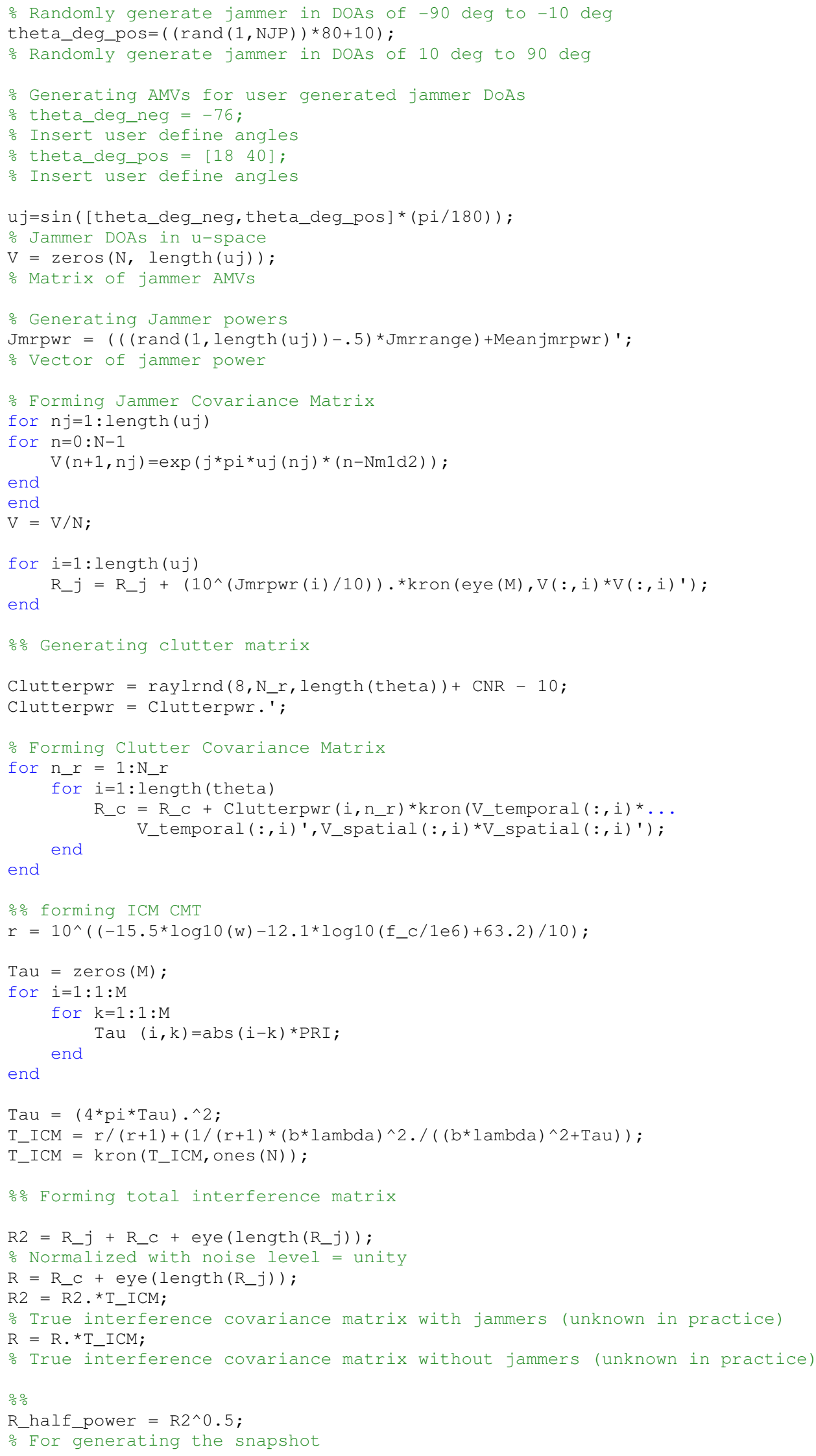




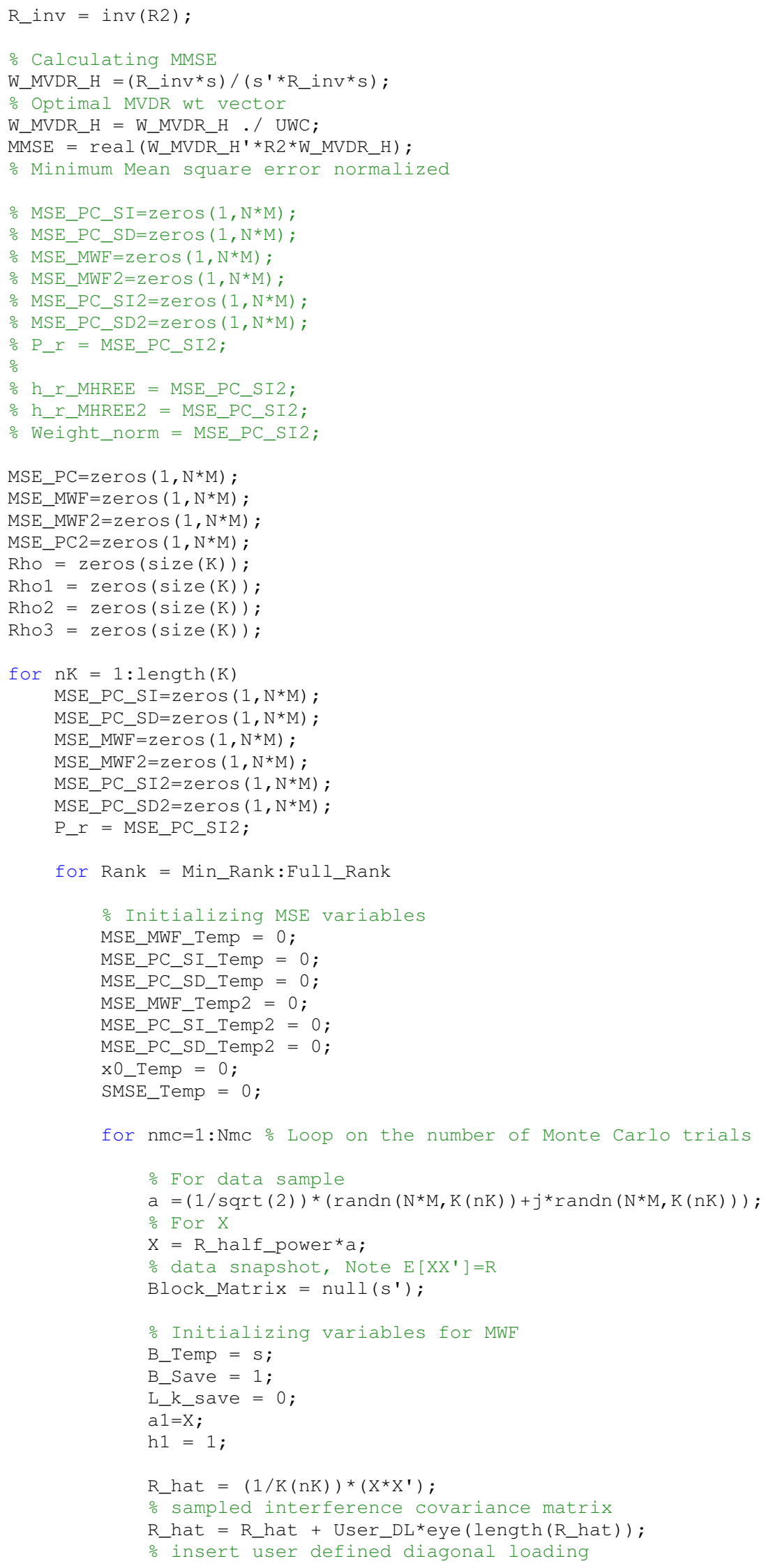




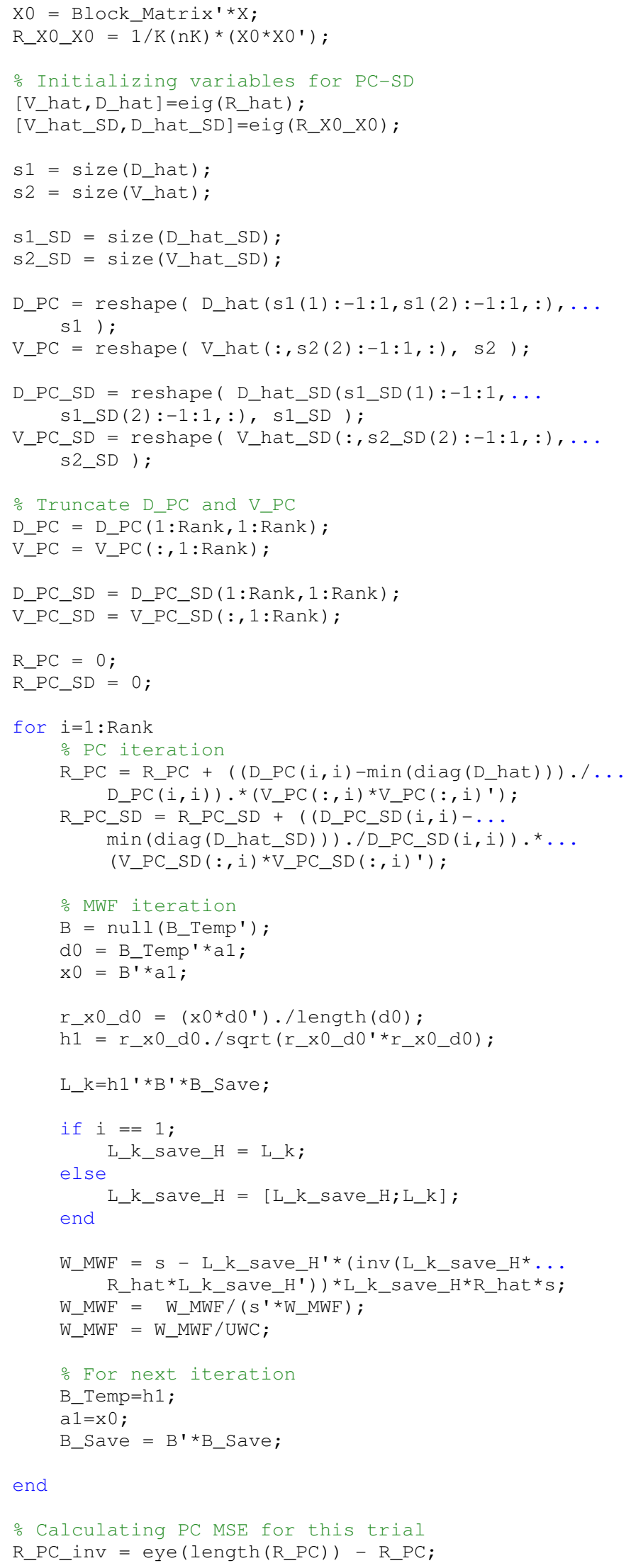




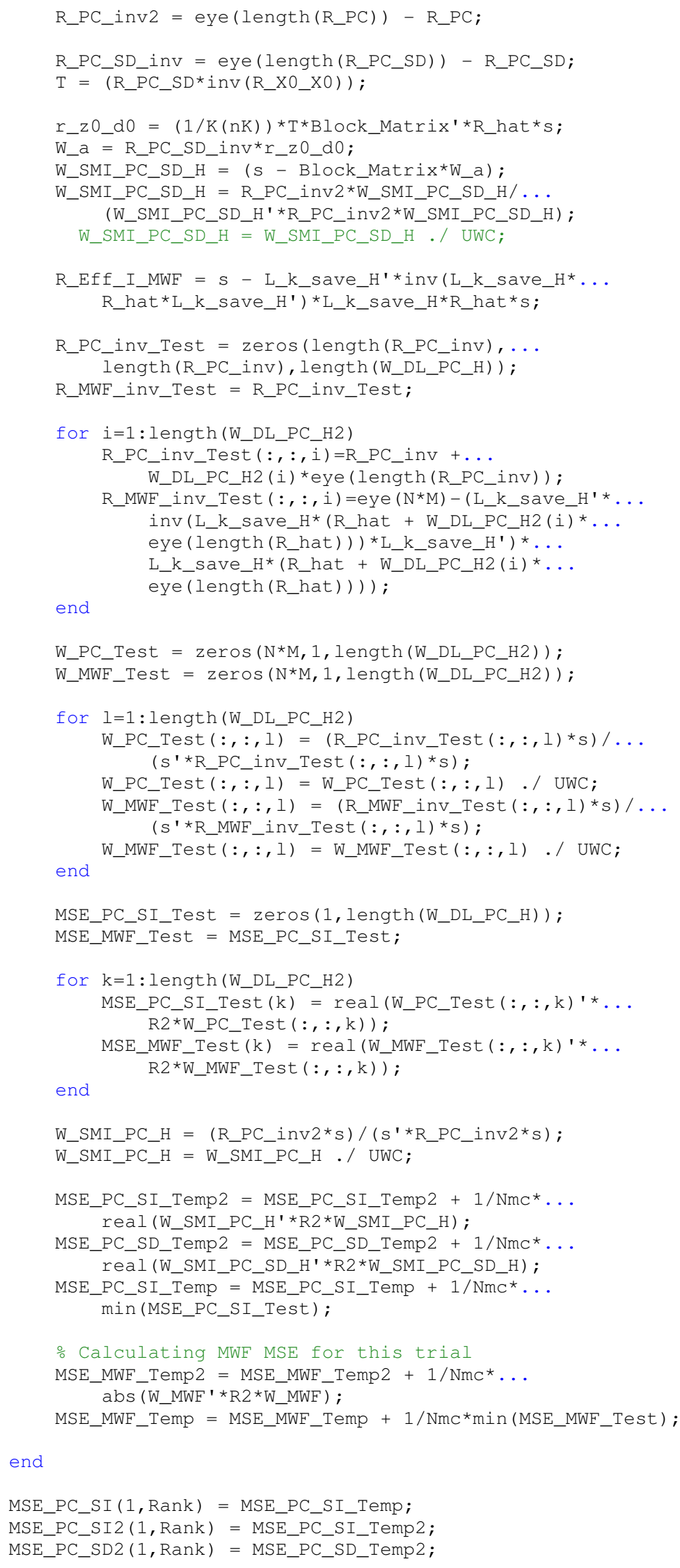




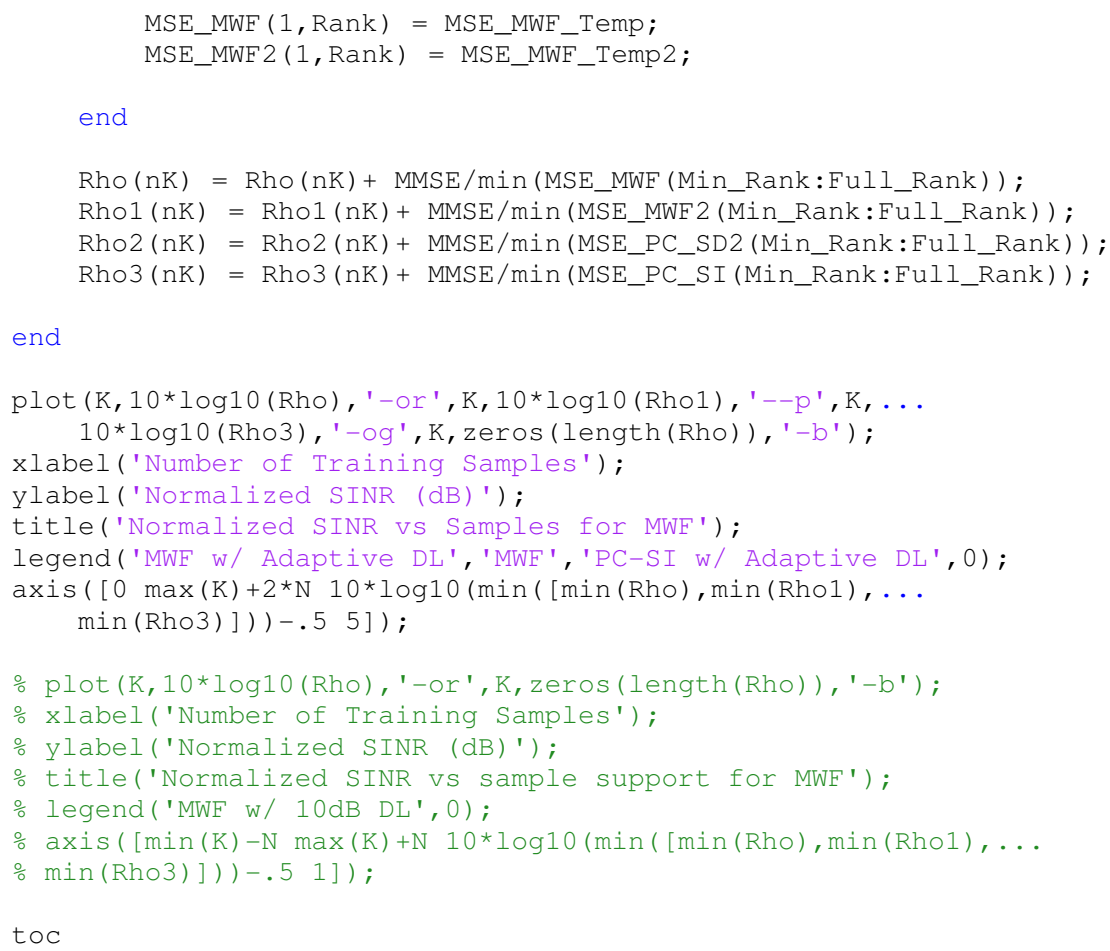




\section{Appendix C: Matlab codes for synthesizing SAR}

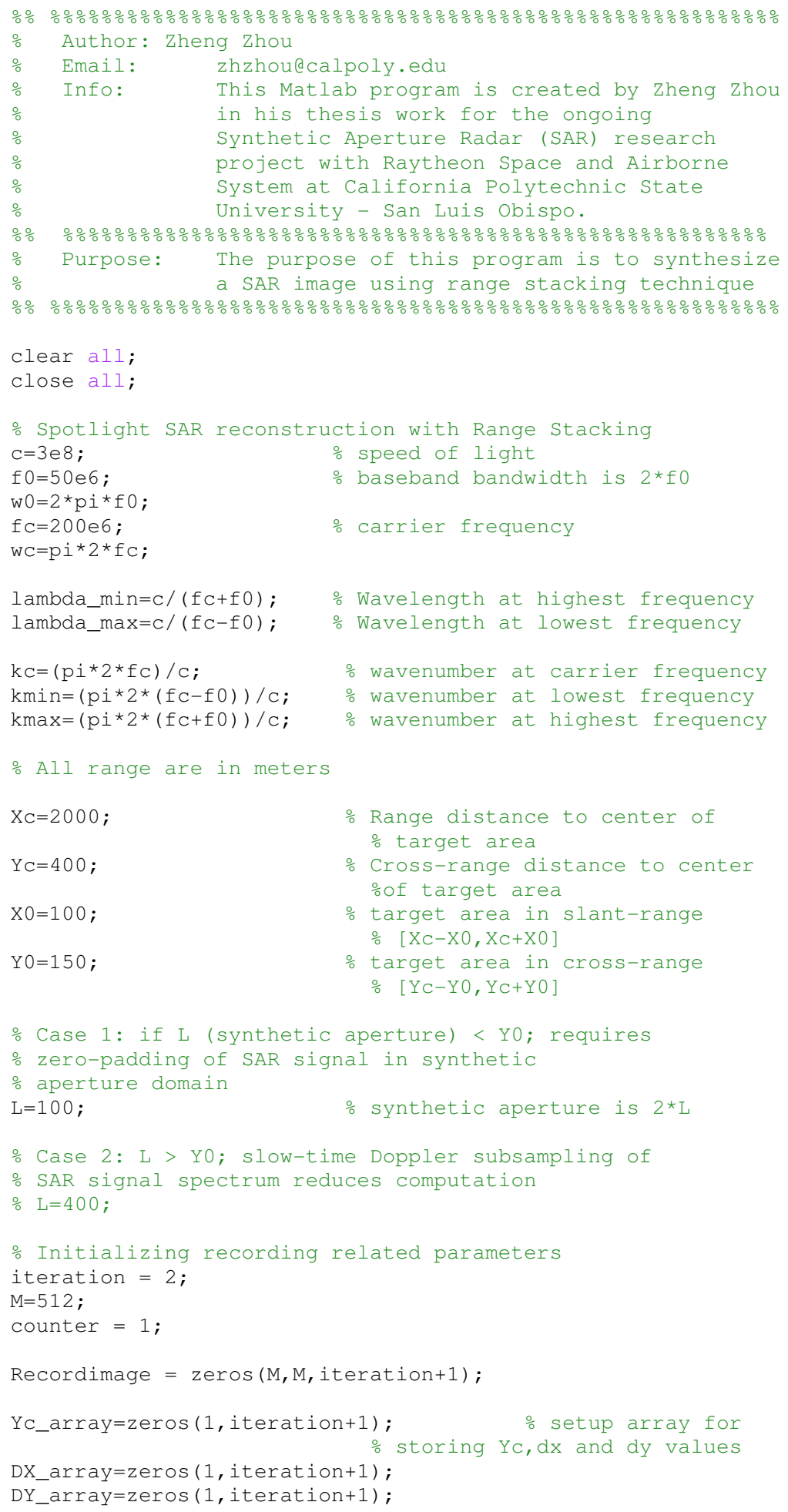




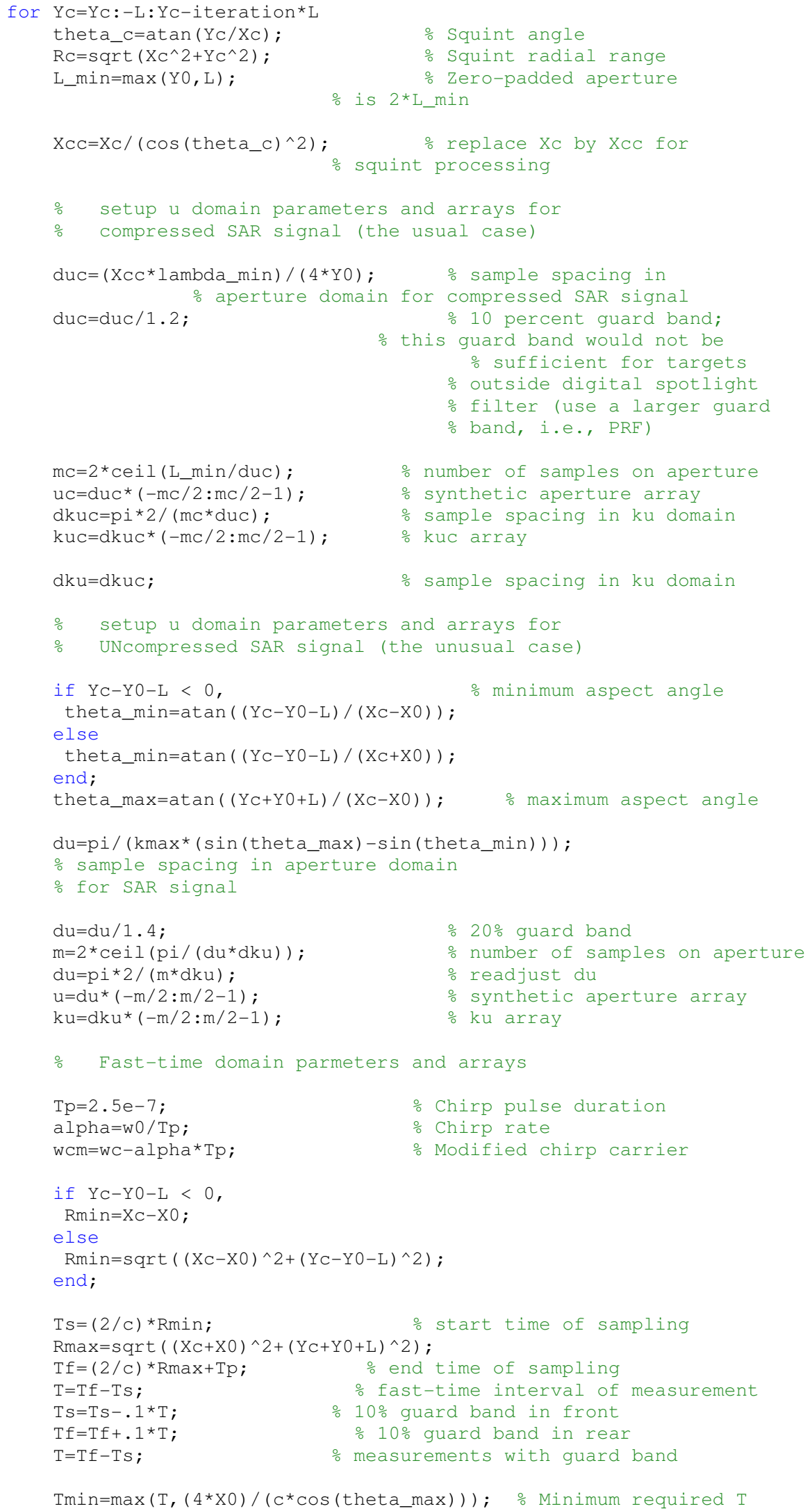




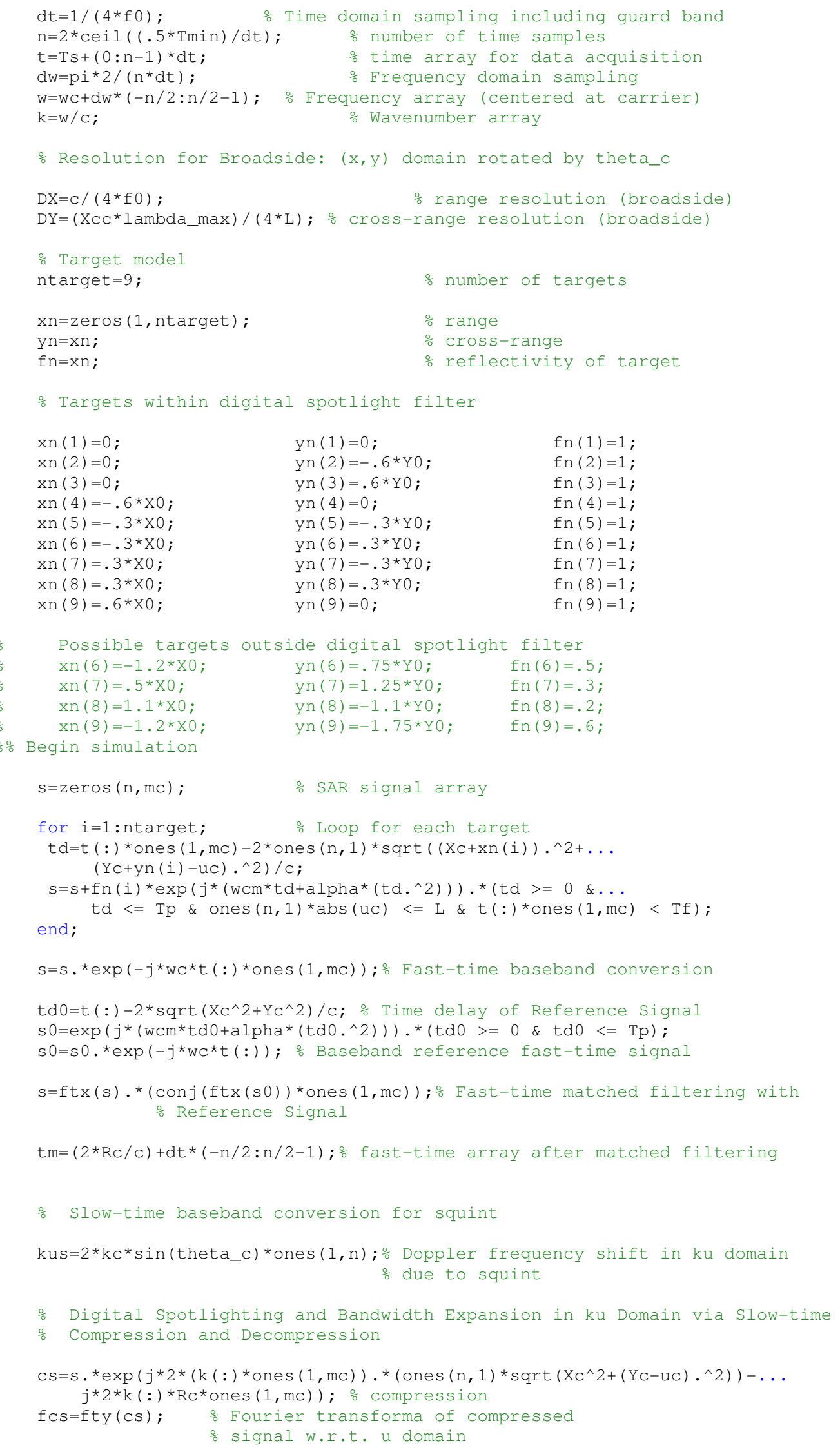




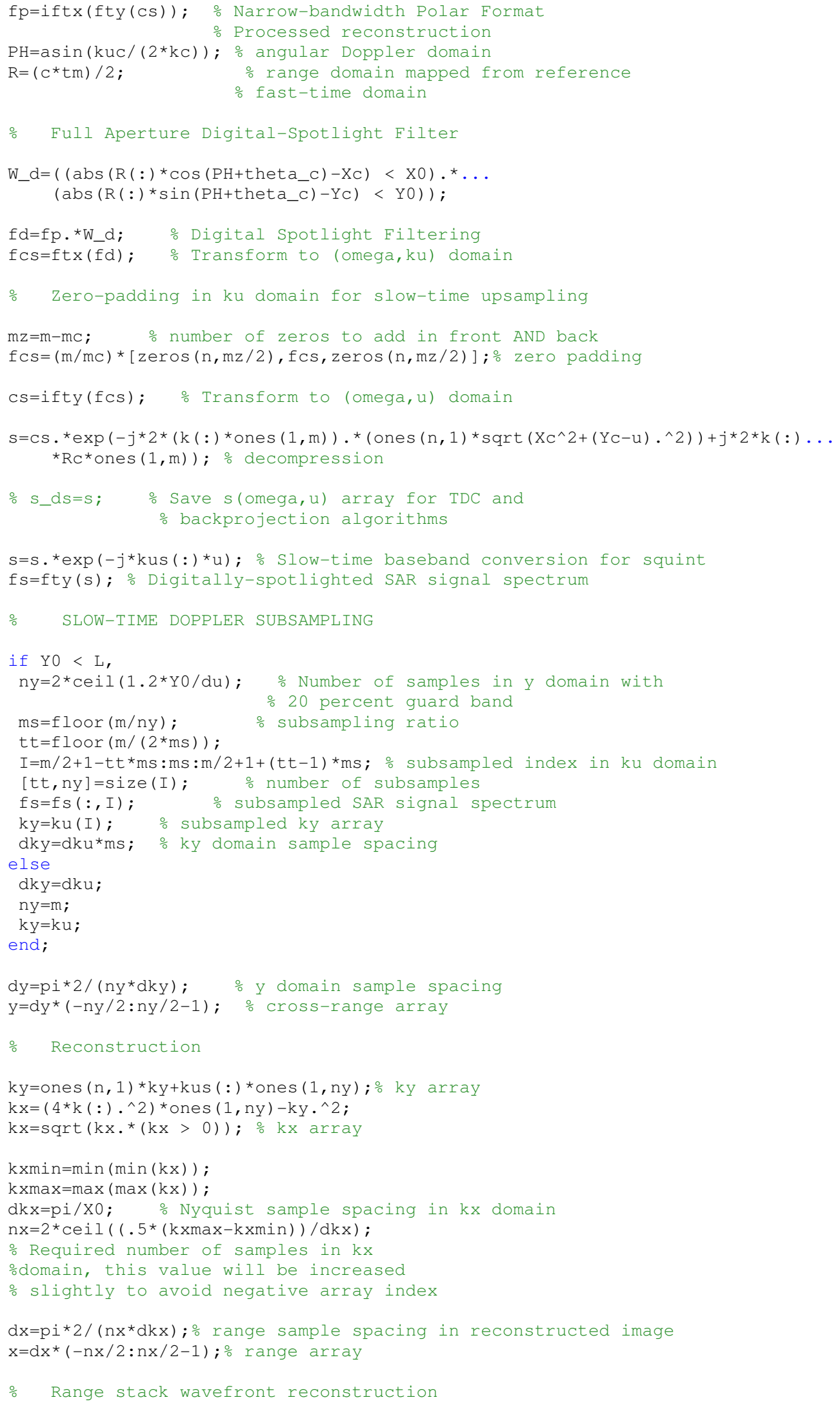




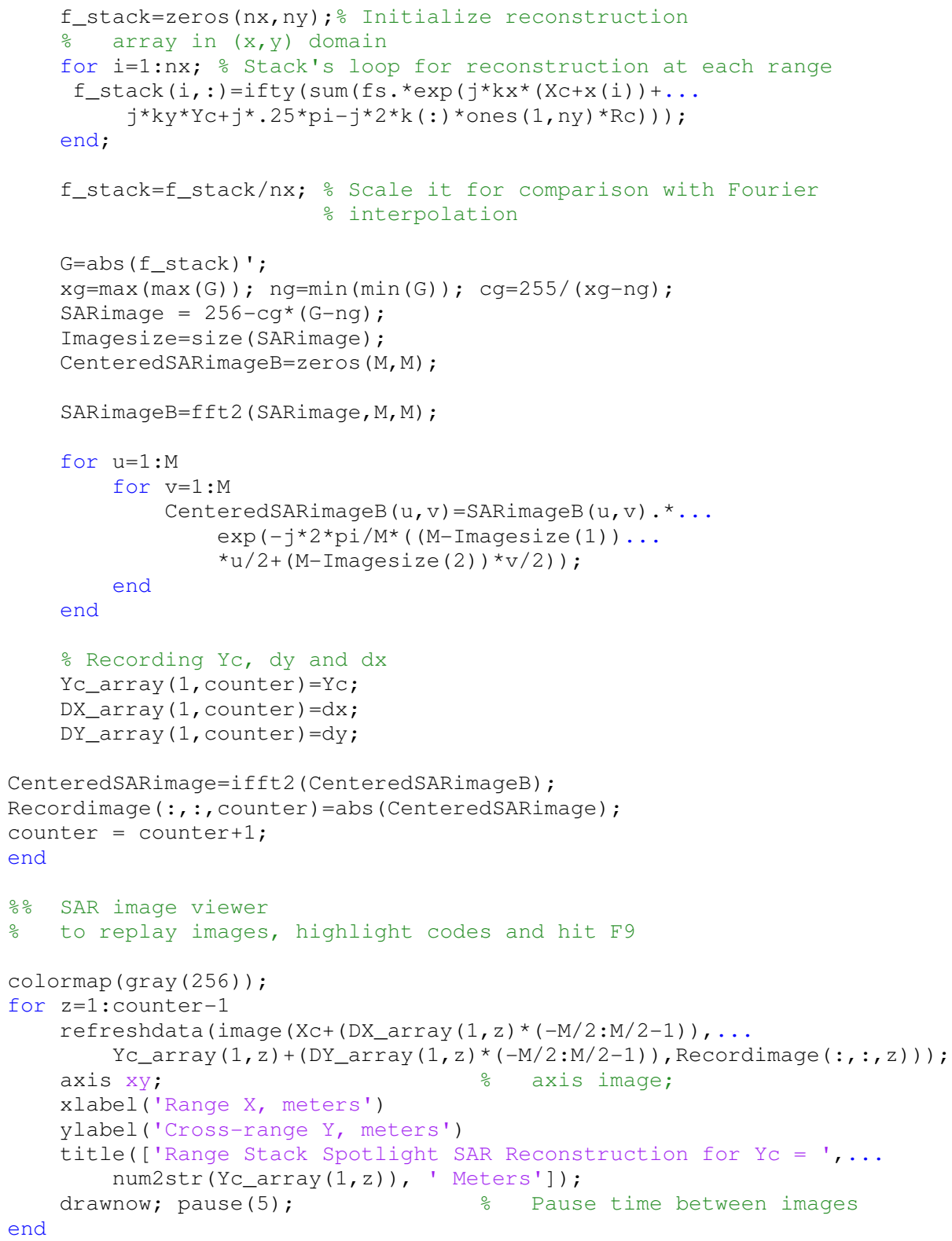




\section{Appendix D: Matlab codes for the studying of CMT effects}

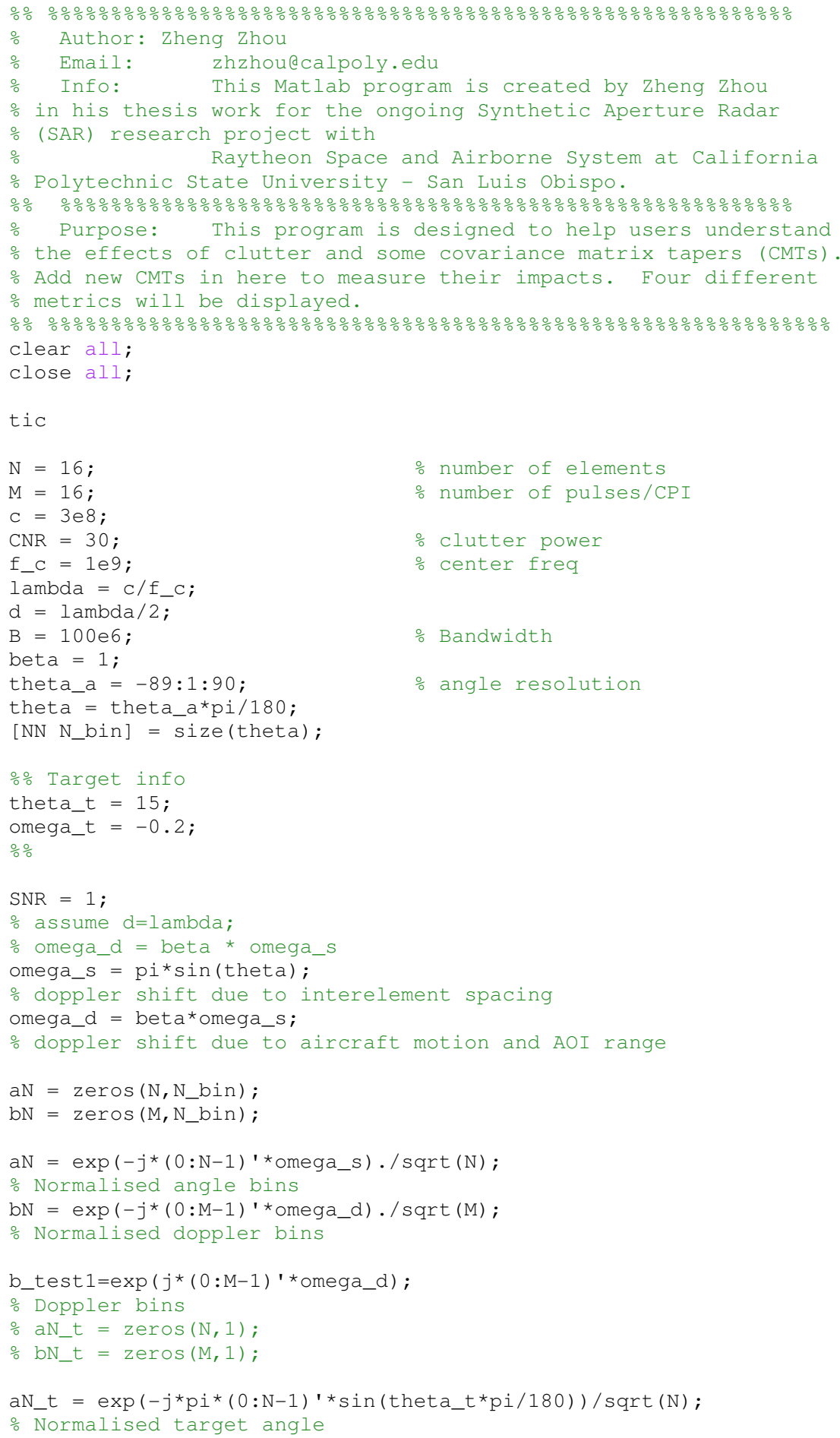




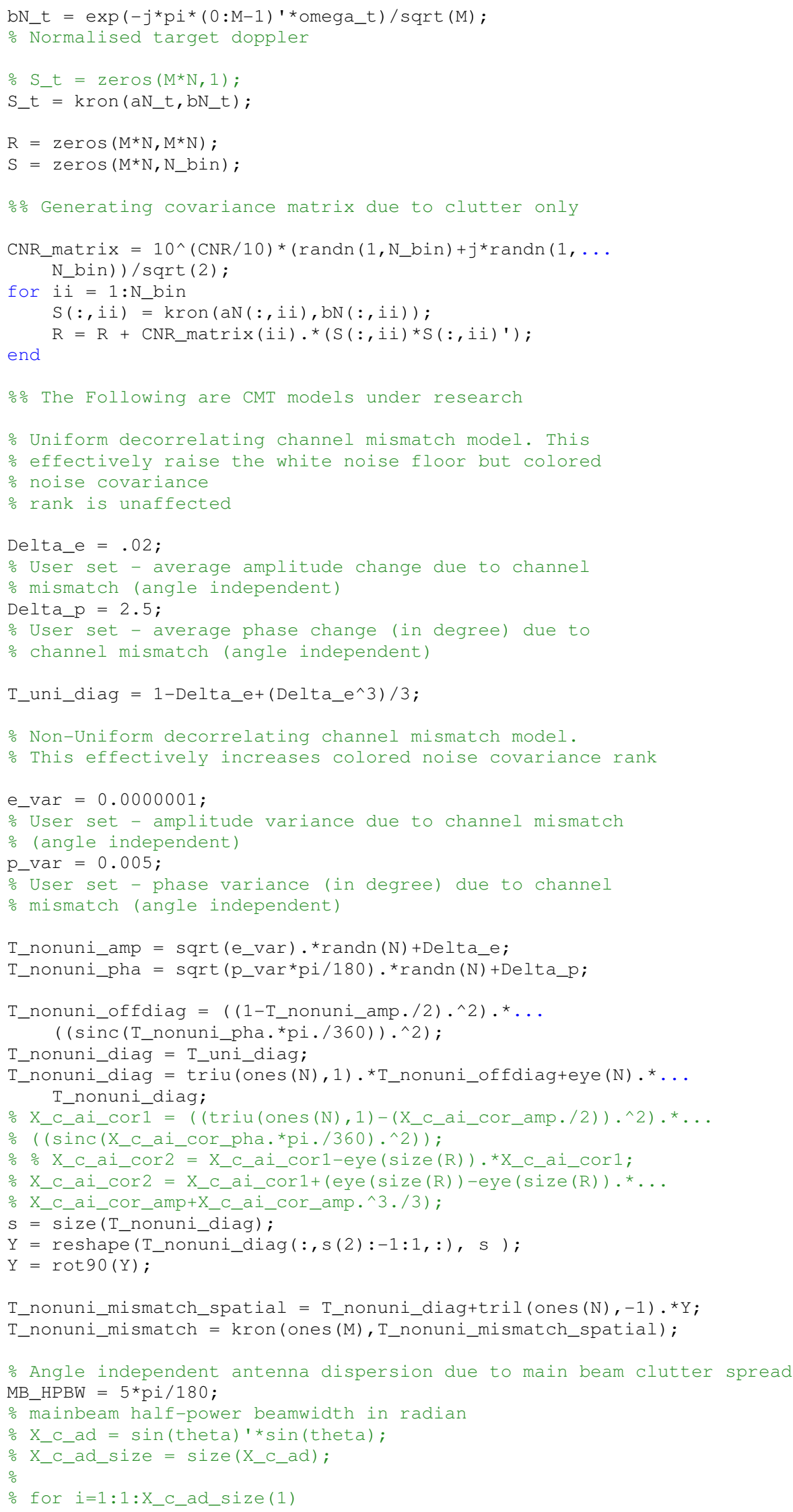




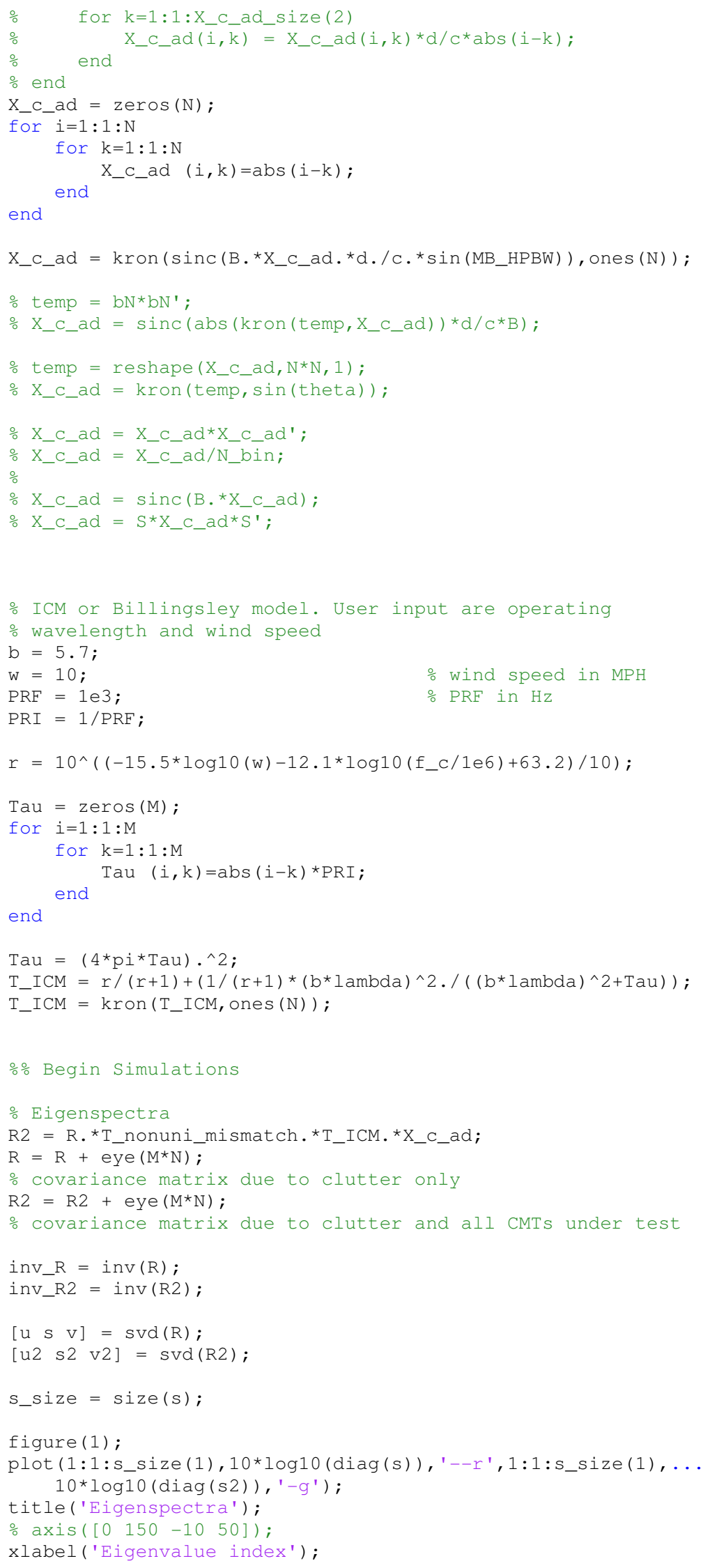




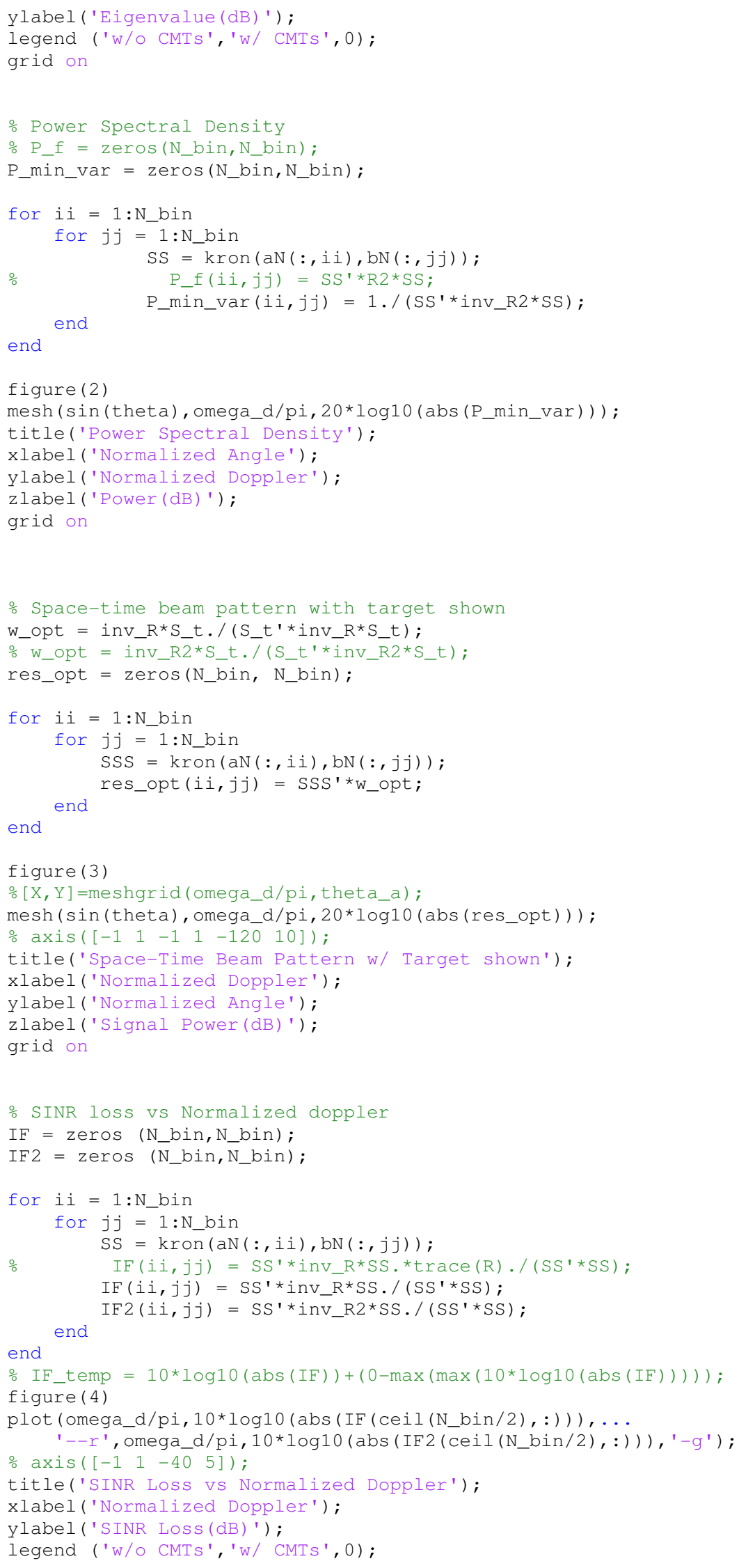




\section{Appendix E: Matlab codes for histogram study of hard stops}

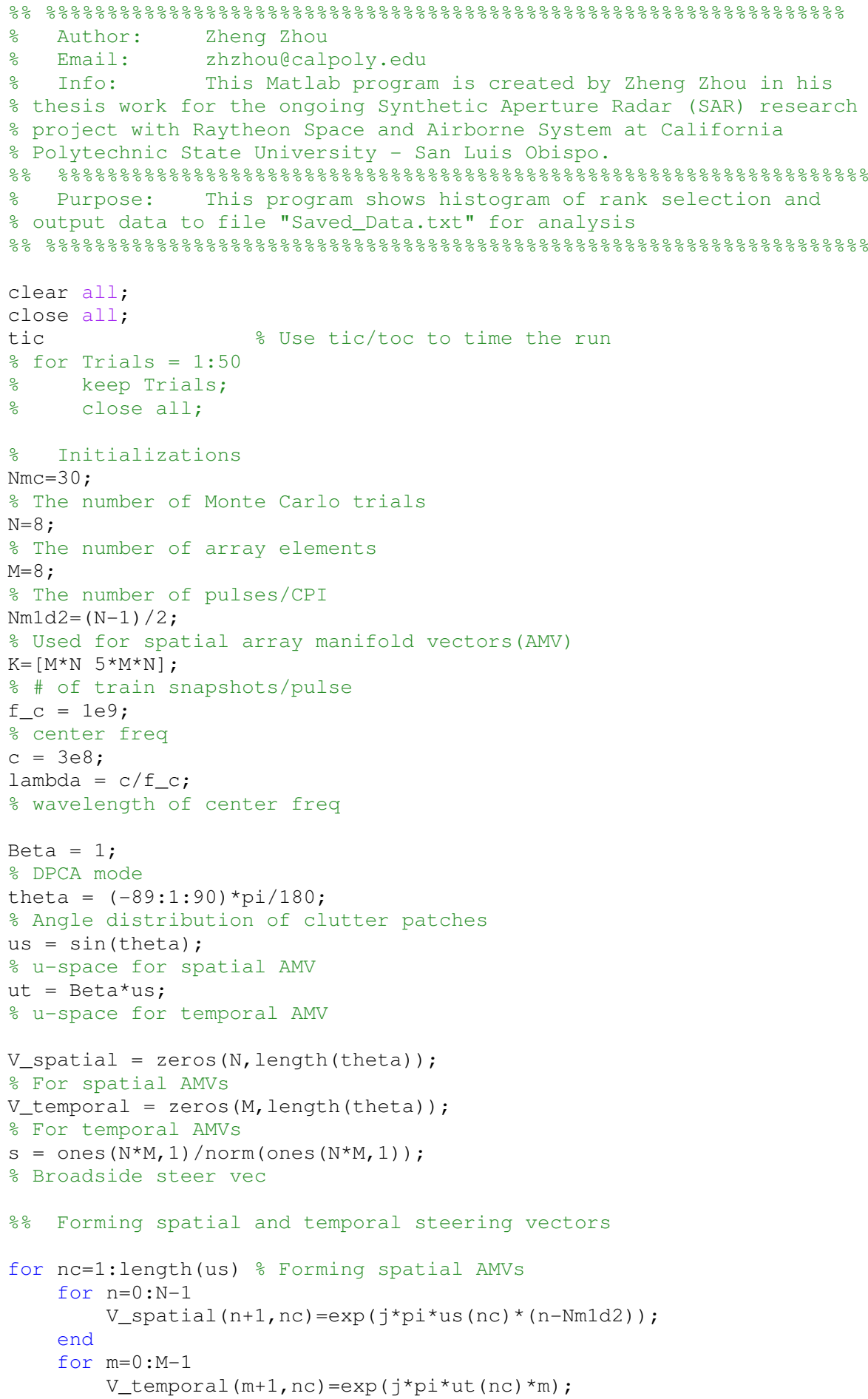




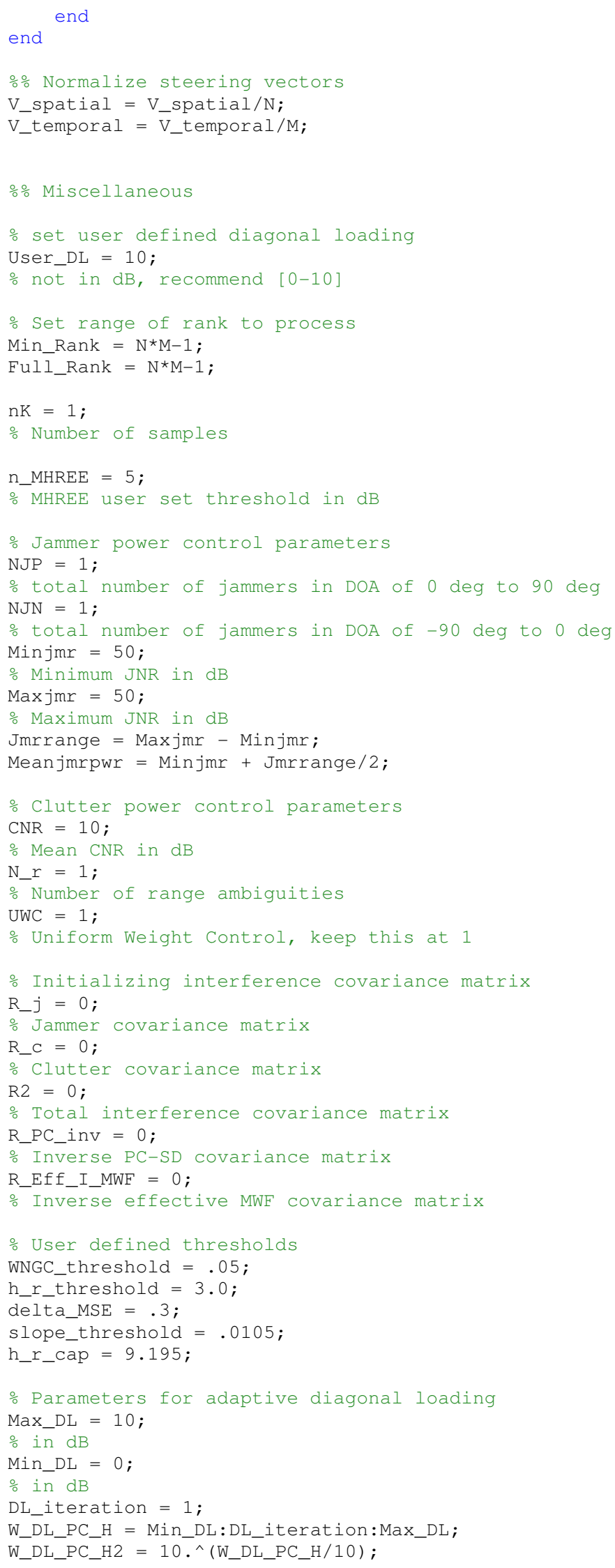




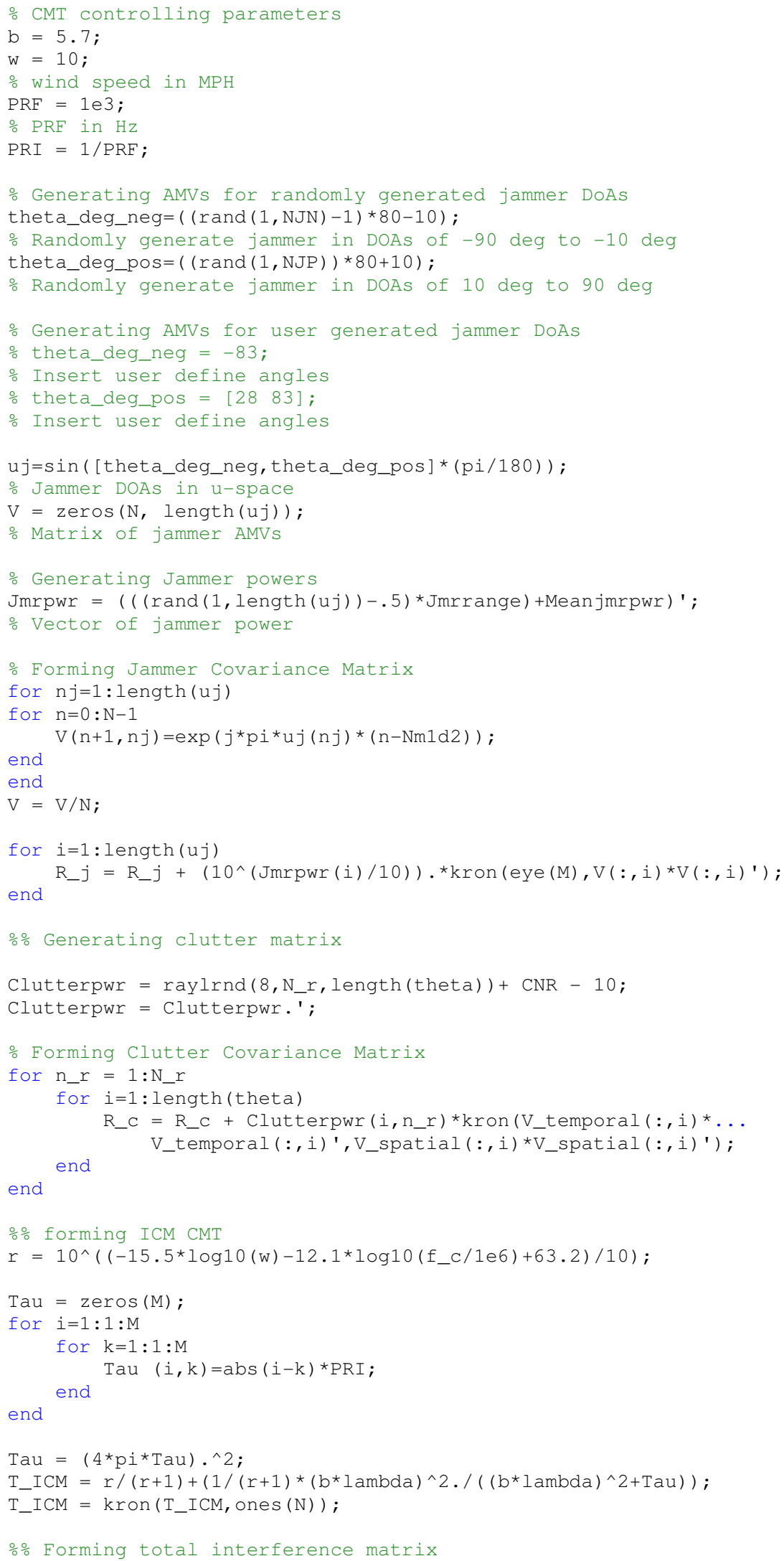




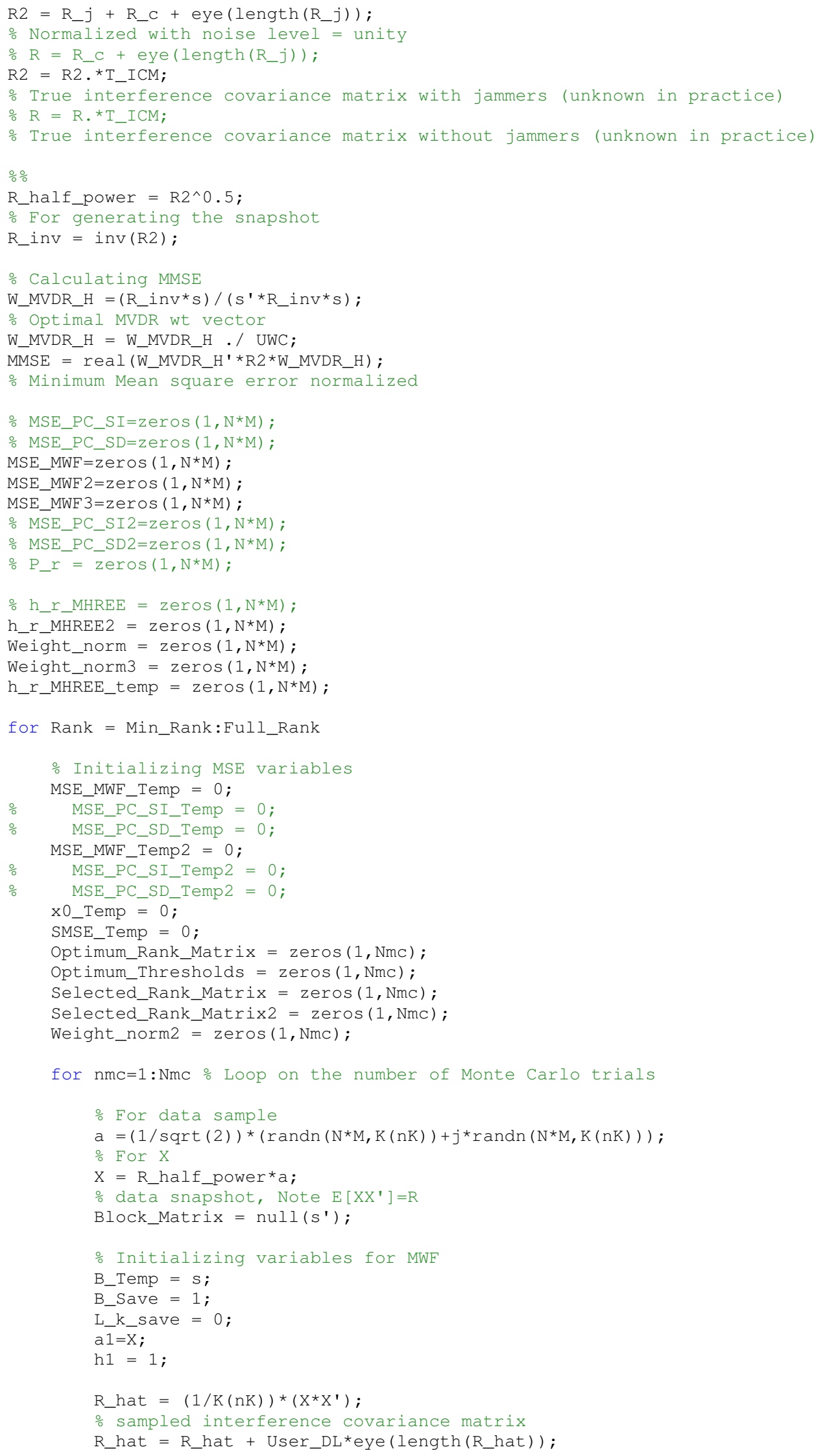


\% insert user defined diagonal loading

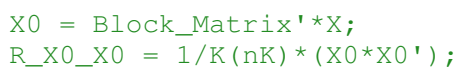




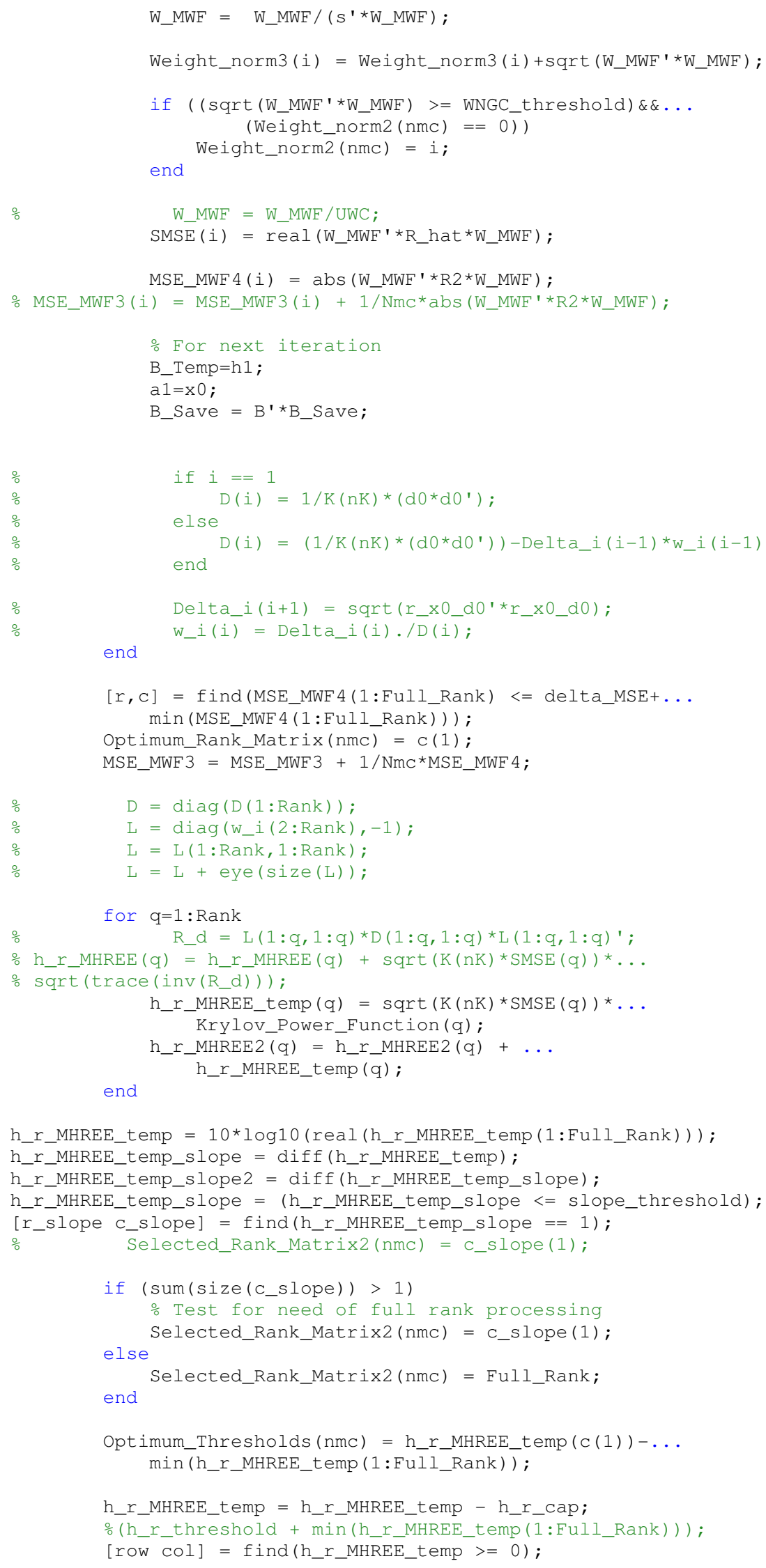




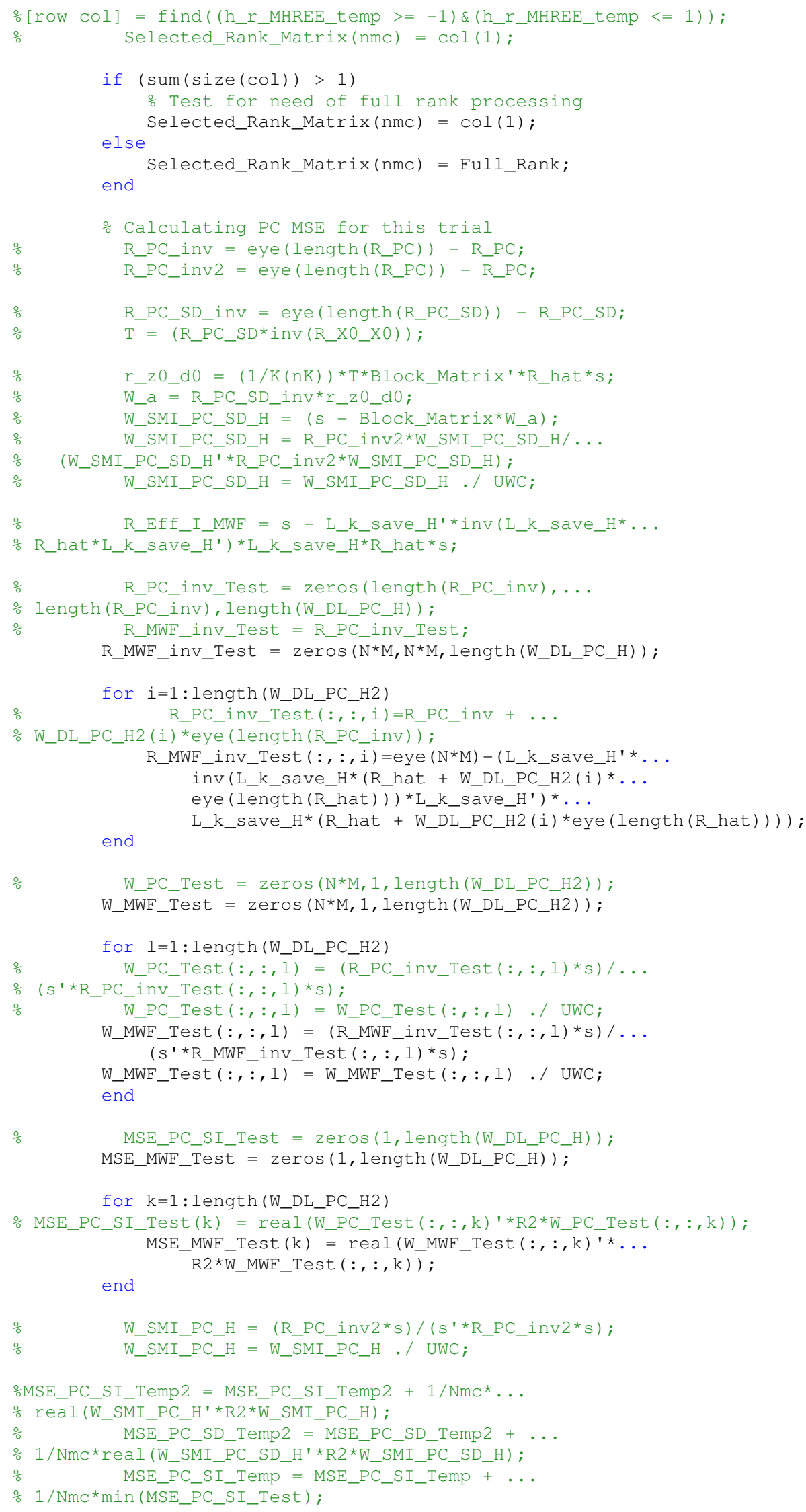




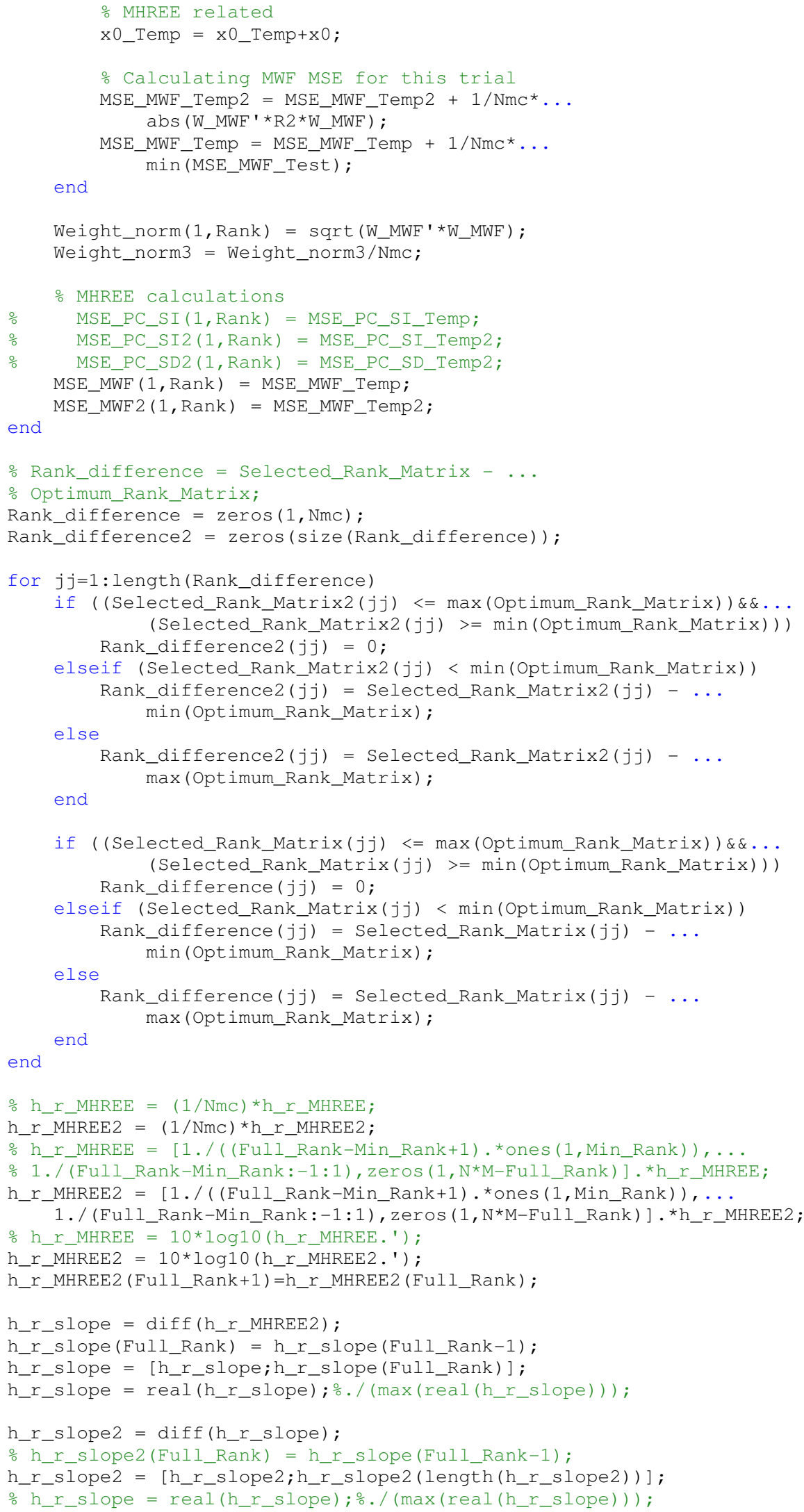




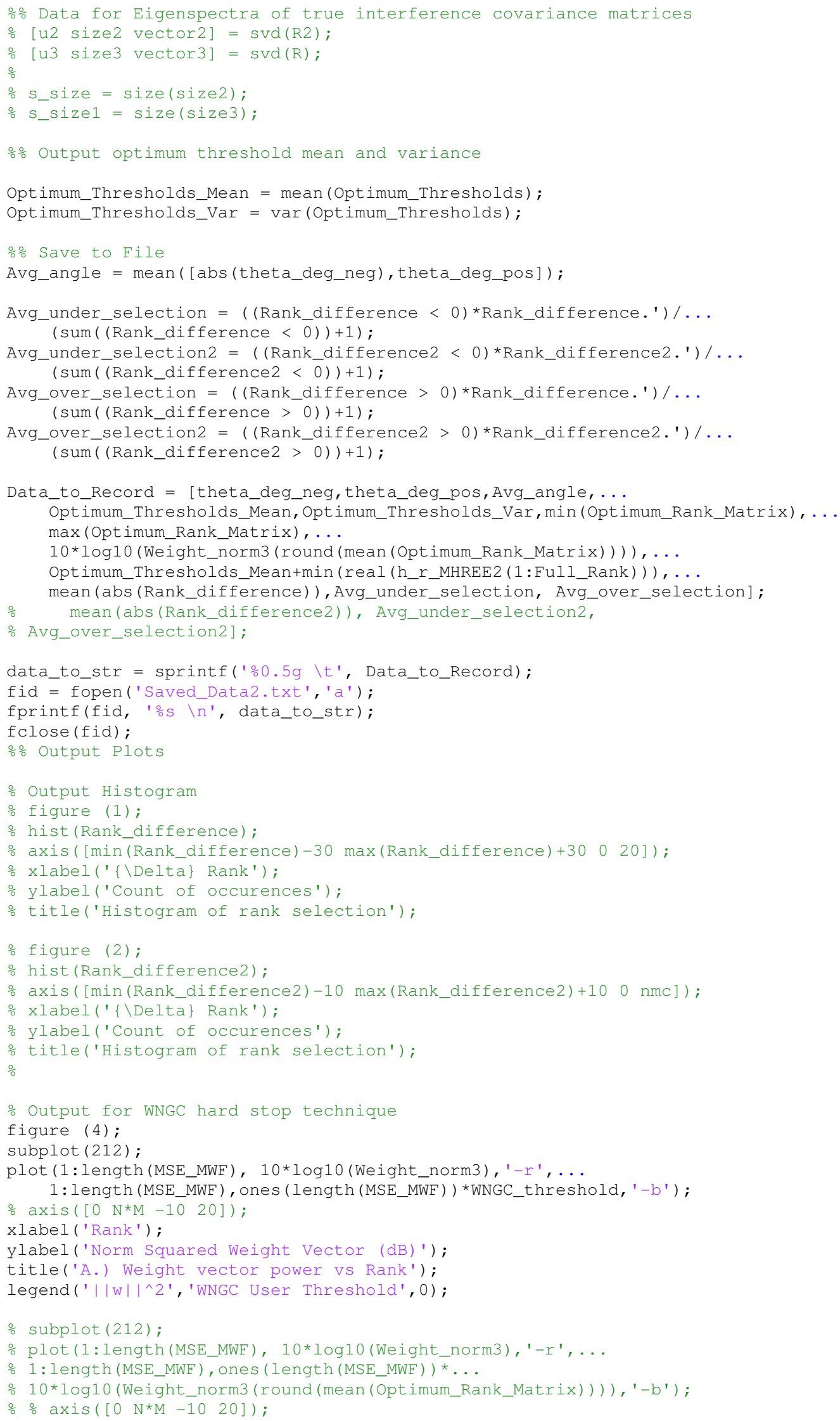




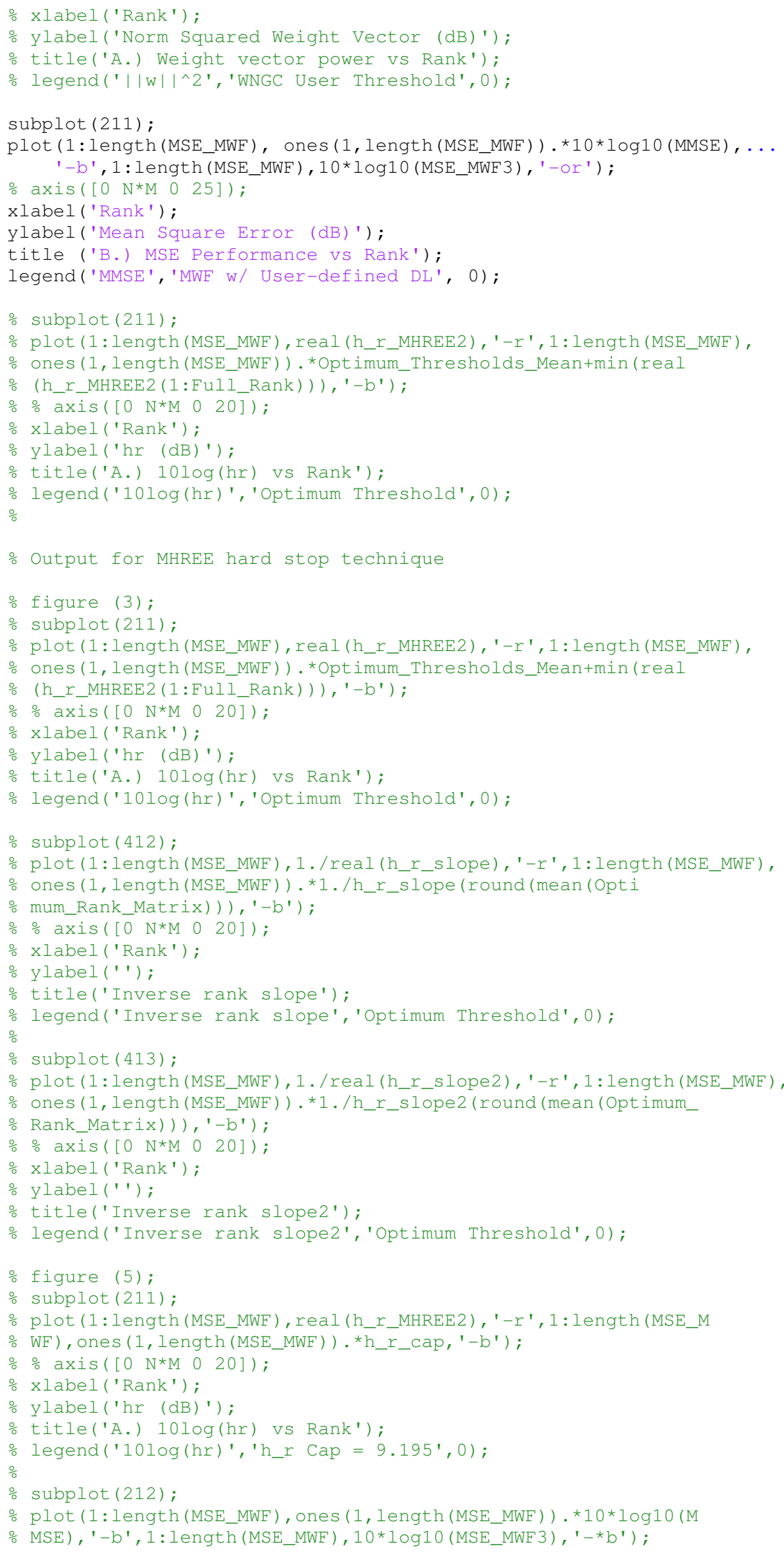


$\therefore$ axis $\left(\left[\begin{array}{llll}0 & N^{*} \mathrm{M} & 0 & 25\end{array}\right]\right)$

\% xlabel ('Rank');

\% ylabel('Mean Square Error (dB)');

o title ('MSE Performance vs Rank');

\% legend('MMSE', 'MWF', 0);

$\div$ end

toc 